\title{
Endothelial Transformation Related Protein 53 Deletion \\ Promotes Angiogenesis and Prevents Cardiac Fibrosis and Heart Failure Induced by Pressure Overload in Mice
}

\section{DOCTORAL THESIS}

In partial fulfillment of the requirements for the degree

"Doctor rerum naturalium (Dr. rer. nat.)"

in the Molecular Medicine Study Program

at the Georg-August University Göttingen

\author{
Submitted by \\ Rajinikanth Gogiraju \\ Born in Mamidimada, India
}

Göttingen, 2014 


\section{Members of the Thesis Committee}

\section{Supervisor}

\section{Prof. Dr. Katrin Schäfer}

University Medical Center Göttingen

Department of Cardiology and Pneumology

Robert-Koch-Strasse 40

37099 Göttingen, Germany

Phone: +49-(0)551 398921

E-mail: katrin.schaefer@med.uni-goettingen.de

\section{Second member of the thesis committee}

\section{Prof. Dr. Matthias Dobbelstein}

Institute of Molecular Oncology

Göttingen Center of Molecular Biosciences, GZMB

Faculty of Medicine, University of Göttingen

Justus von Liebig Weg 11, Room 3.234

37077 Göttingen, Germany

Phone: +49-(0)551 3913840

E-mail: mdobbel@gwdg.de

Third member of the thesis committee

Prof. Dr. Michael Kessel

Max Planck Institute for Biophysical Chemistry

Department of Developmental Biology

Am Fassberg 11

37077 Göttingen, Germany

Phone: +49-(0)551 2011560

E-mail: mkessel1@gwdg.de 
DEDICATED TO MY FAMILY 


\section{Affidavit}

I hereby declare that my doctoral thesis entitled "Endothelial Transformation Related Protein 53 Deletion Promotes Angiogenesis and Prevents Cardiac Fibrosis and Heart Failure Induced by Pressure Overload in Mice" that the thesis has been written independently and with no other sources and aids than quoted.

Rajinikanth Gogiraju

Göttingen, August 2014 


\section{Contents}

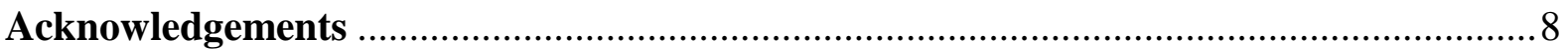

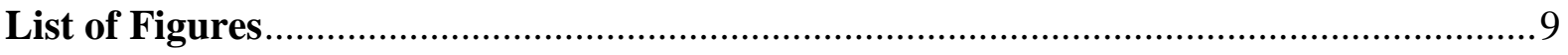

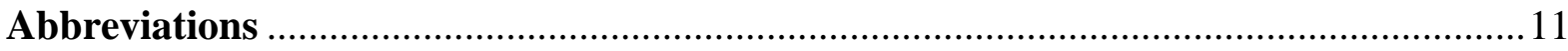

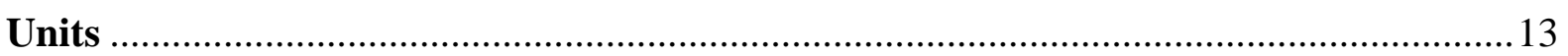

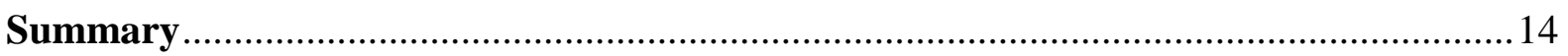

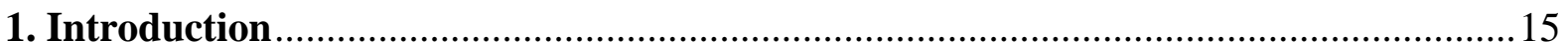

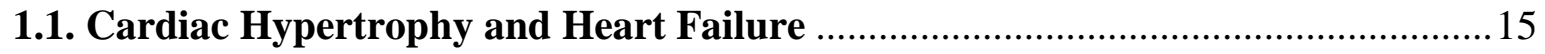

1.1.1. Morphologic Classification of Cardiac Hypertrophy …................................. 15

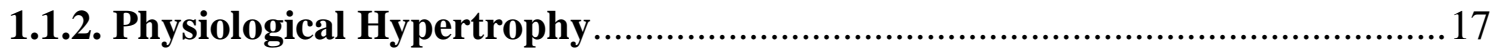

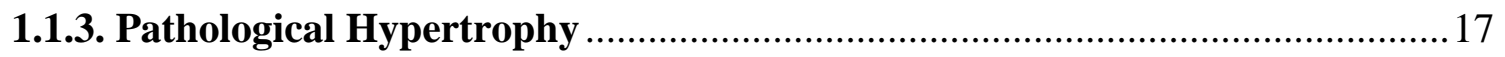

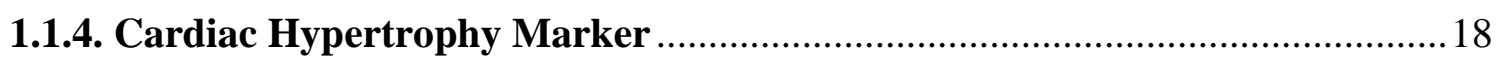

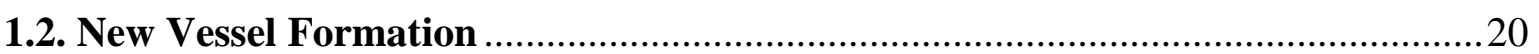

1.2.1. Vascular Endothelial Growth Factors and Receptors...................................22

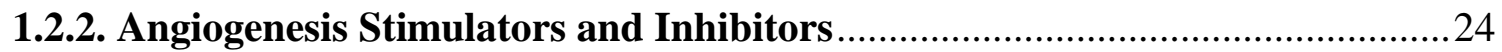

1.3. Tumor Suppressor Protein p53 in Angiogenesis and Heart Failure ....................25

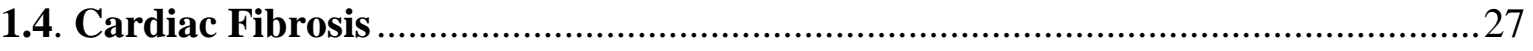

1.4.1. The Origin of Cardiac Fibroblasts during Embryonic Development ............27

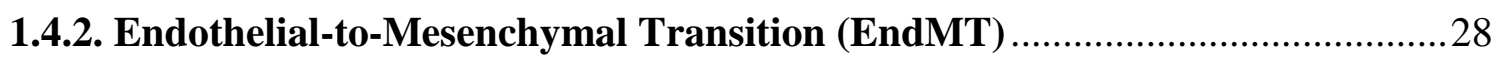

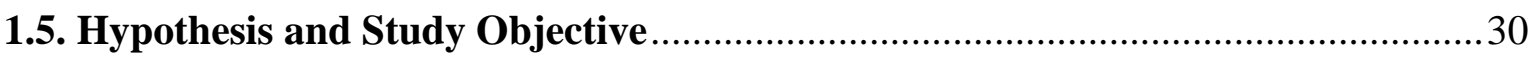

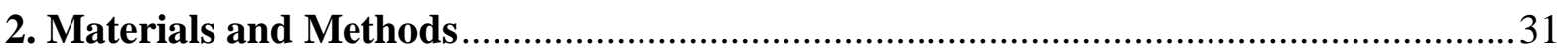

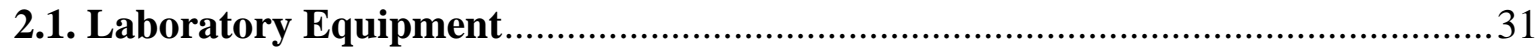

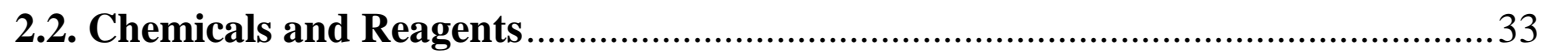

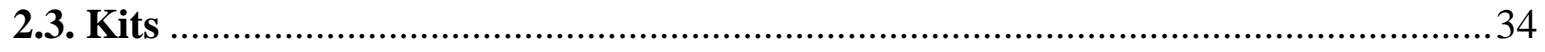

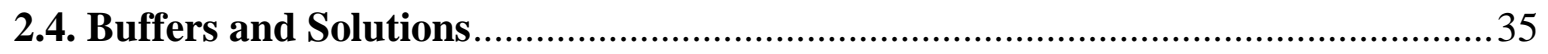

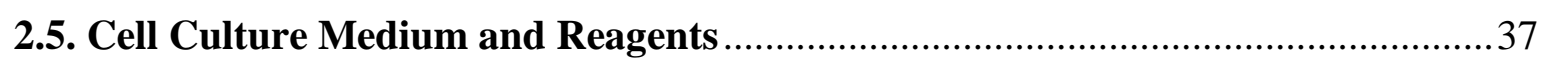

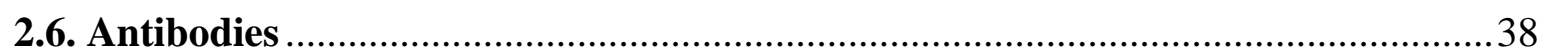

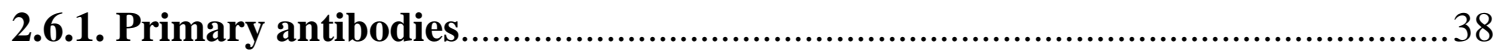

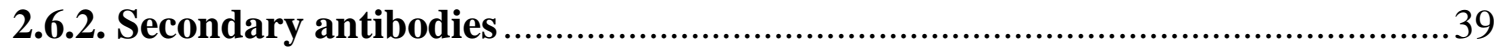

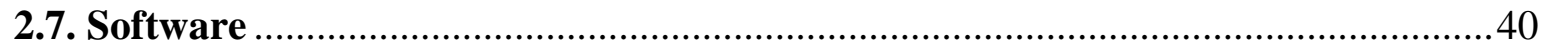

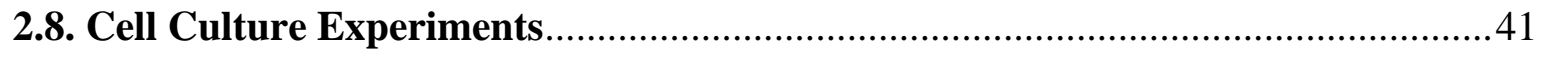

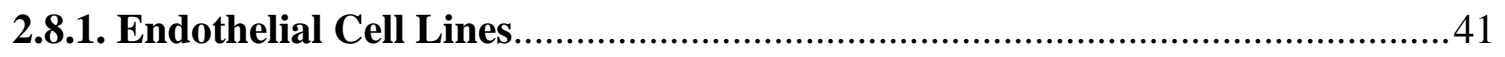

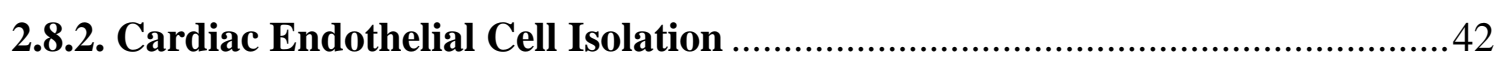

2.8.3. Generation of Stable p53 Knockdown Cell Line.............................................. 43

2.8.4. Modulation of p53 and Induction of EndMT in vitro ....................................43

2.8.5. Endothelial Cell Functionality Assays ......................................................... 44

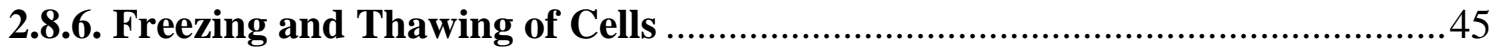


2.9. Experiments in Mice .46

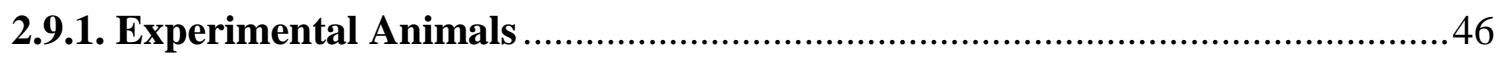

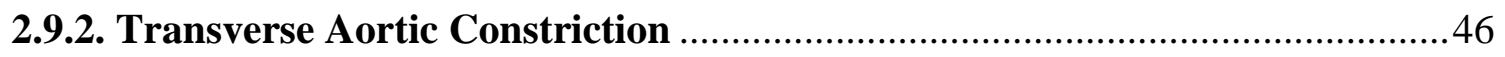

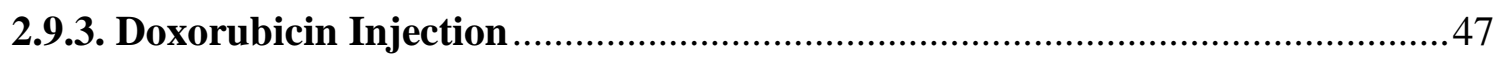

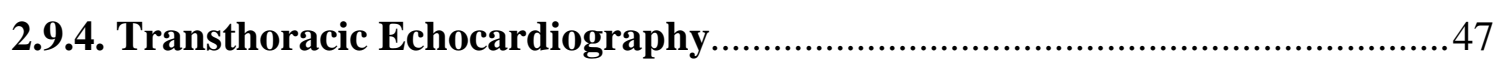

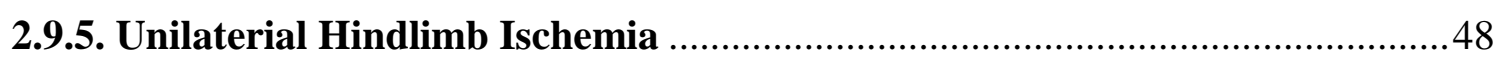

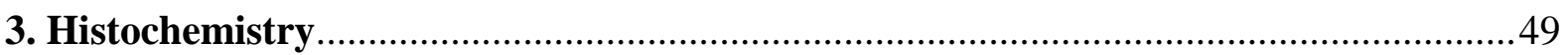

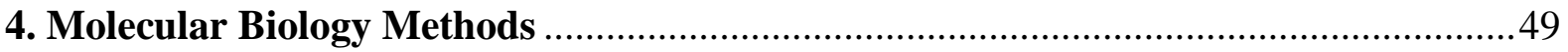

4.1. Genomic DNA Isolation from Mouse Tail ............................................................. 49

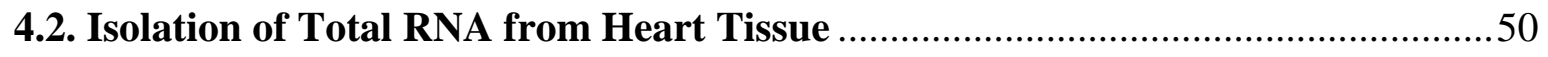

4.3. Determination of RNA Quality and Concentration..........................................51

4.4. Reverse Transcription Polymerase Chain Reaction (RT-PCR) ............................51

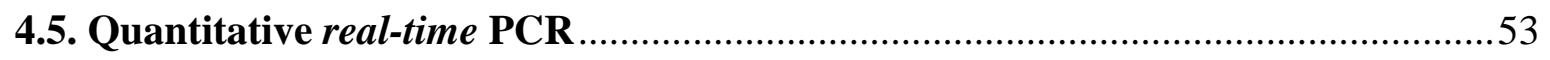

4.6. Agarose Gel Electrophoresis Detection of PCR Amplicons .................................55

4.7. Primer Sequences and qRT-PCR Conditions .......................................................56

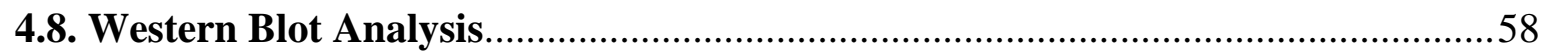

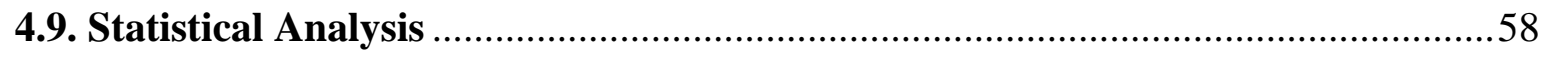

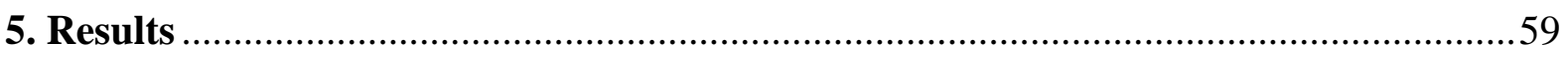

5.1. Generation of Endothelial-Specific p53 Knockout Mice .....................................59

5.2. Endothelial p53 Deletion Protects Against Cardiac Hypertrophy and Dysfunction

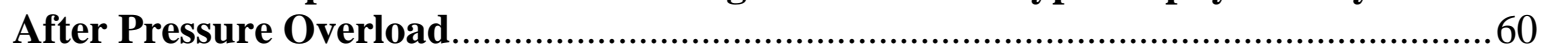

5.3. Endothelial p53 Deletion Reduces Cardiac p53 Accumulation in Endothelial and Non-Endothelial Cells after TAC

5.4. Endothelial p53 Deletion Reduces Apoptosis in Endothelial and Non-Endothelial Cells After TAC.

5.5. Deletion of p53 in Endothelial Cells is Associated with Higher Cardiac Hif1 $\alpha$ and Vegf Expression.

5.6. Deletion of p53 in Endothelial Cells Promotes Cardiac Angiogenesis ..................71

5.7. Endothelial p53 Deletion Reduces Hypoxia in Hearts After TAC ....................... 73

5.8. Endothelial p53 Deletion Promotes Extra-Cardiac Angiogenesis ........................ 74

5.9. Inactivation/ Inhibition of p53 Activity Improves Angiogenic Properties of

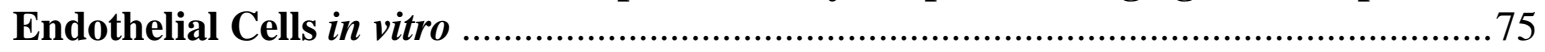

5.10. Hearts from End.p53-KO Mice Revealed Lower Levels of Apoptosis and Improved Angiogenesis After Doxorubicin Treatment .............................................. 77

5.11. Endothelial p53 Deletion Attenuates Pressure Overload-Induced Cardiac Fibrosis .78

5.12. Deletion of p53 in Endothelial Cells Reduces Surrogate Markers of Endothelial-

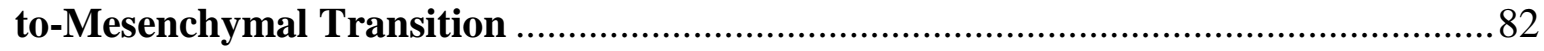

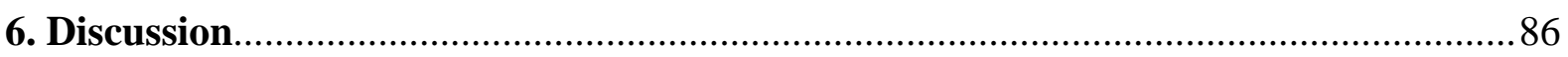




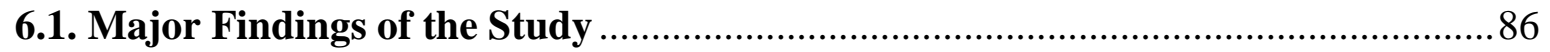

6.2. Role of p53 in Myocardial Apoptosis and Heart Failure …................................. 86

6.3. Deletion of p53 in Endothelial Cells Exhibit Less Apoptosis and Hypoxia ...........88

6.4. Role of Hypoxia in Cardiac Hypertrophy and Angiogenesis.................................8 89

6.5. Deletion of p53 Specifically in Endothelial Cells Enhances Cardiac and ExtraCardiac Angiogenesis and Postponed Heart Failure after TAC .............................90

6.6. Deletion of p53 Specifically in Endothelial Cells Attenuates TAC Induced Cardiac Fibrosis and Endothelial-Mesenchymal Transition (EndoMT) ....................91

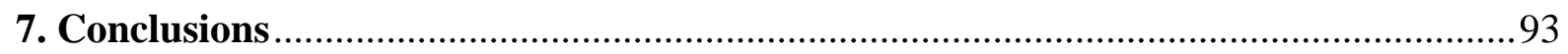

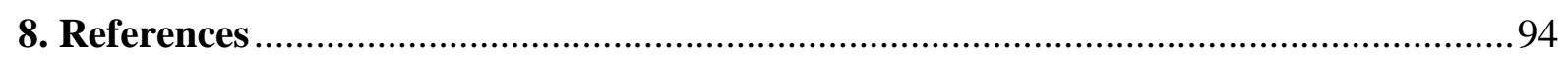

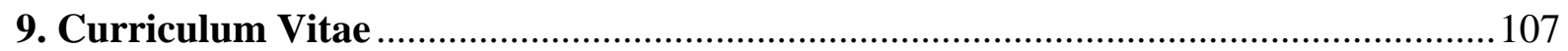




\section{Acknowledgements}

First of all, I would like to express my sincere gratitude to my supervisor, Prof. Dr. Katrin Schaefer, for giving me an opportunity to do my Ph.D. study in her research group and providing an excellent working atmosphere. Her guidance helped me all the time of my research and writing of this thesis.

Besides my supervisor, I would like to thank the rest of my thesis committee members Prof. Dr. Matthias Dobbelstein and Prof. Dr. Michael Kessel for their valuable suggestions, comments and support during my PhD study.

I specially thank Prof. Dr. Wolfram-Hubertus Zimmermann, Prof. Dr. Michael Zeisberg and Prof. Dr. Susanne Lutz for accepting my request to be the external examiners for my thesis defense. I would also like to thank Prof. Dr. Elisabeth Zeisberg and her group members Xingbo $\mathrm{Xu}$ and Xiaopeng Liu for their collaboration and contribution in our project.

I would like to thank Kirsten Koschel, Julia H. Steinbrecher, Sarah Zafar, Celina Fraatz, Anika Hunold, Sarah Barke, for their technical assistance. I would like to thank all the lab members, Dr. Marco R. Schroeter, Dr. Frauke Czepluch, Dr. Maren Leifheit-Nestler, Astrid Hubert, Norman Eschholz, Sebastian Herzberg, Markus Bernhardt, Hendrik Kuschicke, Magdalena Schlegel and Julia Meier for the excellent working environment in the lab during the last four years.

Last but not the least, I would like to thank my nana and amma, G. Venkateswar Rao and G. Bhagya Laxmi, for giving me the beautiful life and supporting me financially and emotionally throughout my life. A special thanks to my beloved wife Naga Jyothi and my two loving daughters Sreshta and Sravya. I also thank my parents-in-law Narsimha Rao, Kalavathamma for their constant support. I would like to thank my pednana G. Anantha Rao, ayamma G. Ranganayakamma, my brothers especially Shyam Sundar Rao and Amarendhar Rao for their guidance, my sisters, my brother-in-laws and my sister-in-laws for their love and caring towards me. 


\section{List of Figures}

Figure 1.1. The heart has the ability to increase its size and, depending on the stimulus, this results in physiological or pathological hypertrophy ............................. 2

Figure 1.2. Physiological Versus Pathological Hypertrophy....................................... 4

Figure 1.3. Venn diagram depicting shared and distinct gene cluster expression profiles of physiological and pathological hypertrophy........................................... 5

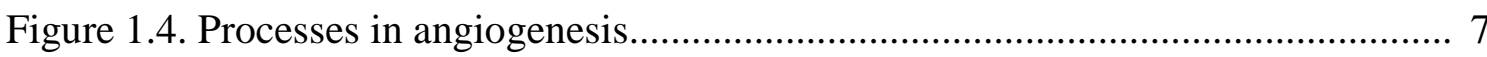

Figure 1.5. VEGF super family ligands and receptors............................................. 9

Figure 1.6. Role of HIF1 $\alpha$ in the expression of VEGF and the stimulation of

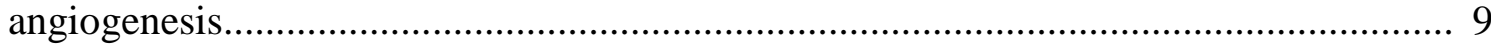

Figure 1.7. Endogenous angiogenesis inhibitors...................................................... 10

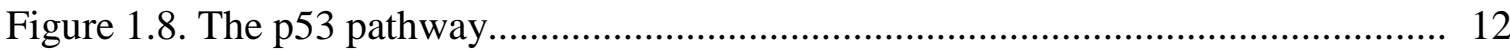

Figure 1.9. Origin and sources of fibroblasts during organ fibrosis............................ 14

Figure 1.10. Schematic diagram illustrating potential mechanisms involved in

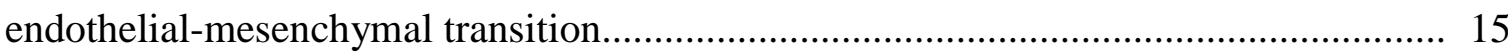

Figure 2.1. Microscopic image of HCMEC and HUVEC........................................... 27

Figure 2.2. Transverse Aortic Constriction.................................................................... 33

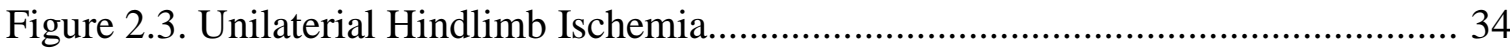

Figure 5.1. Genotyping of endothelial p53 knockout and wildtype mice...................... 45

Figure 5.2. Flow cytometry of Tie2- and CD45-positive cells isolated from blood,

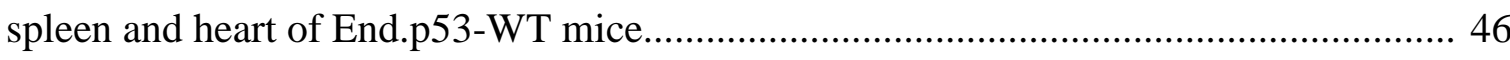

Figure 5.3. Effect of endothelial p53 deletion on survival......................................... 47

Figure 5.4 Effect of endothelial p53 deletion on cardiac hypertrophy and function...... 48

Figure 5.5. Effect of endothelial p53 deletion on cardiac hypertrophy.......................... 49

Figure 5.6. mRNA expression of p53 and p53-regulated factors in banded mouse hearts

Figure 5.7. Protein expression of p53 and p53-regulated factors in banded mouse hearts

Figure 5.8. Reduced number of p53 positive endothelial and non endothelial cells in End.p53-KO mice. 52

Figure 5.9. Effects of endothelial p53 deletion on caspase-3 levels after 53 TAC.

Figure 5.10. Effects of endothelial p53 deletion on cardiac apoptosis after TAC......... 54 
Figure 5.11. Role of endothelial p53 for the cardiac and endothelial response to hypoxia at 8 and 20 weeks after TAC.

Figure 5.12. Role of endothelial p53 for the cardiac and endothelial response to hypoxia after 7 days TAC

Figure 5.13. Deletion of p53 in endothelial cells induces HIF1 $\alpha$ expression when exposure to chemical hypoxia 57

Figure 5.14. Cardiac vascularization in End.p53-WT and End.p53-KO mice 58

Figure 5.15. Expression of hypoxic marker in End.p53-WT and End.p53-KO mice.

Figure 5.16. Effect of endothelial p53 deletion on neovascularization following hindlimb ischemia.

Figure 5.17. Effect of nutlin-3a and pifithrin- $\alpha$ treatment on p53 modulation of endothelial cells in vitro.

Figure 5.18. Effect of p53 modulation on the angiogenic properties of endothelial cells in vitro.

Figure 5.19. p53 expression, apoptosis and angiogenesis after doxorubicin treatment in vitro and in vivo.

Figure 5.20. Apoptosis and angiogenesis after doxorubicin treatment in vitro and in vivo. 64

Figure 5.21. Effect of endothelial p53 deletion on cardiac fibrosis 65

Figure 5.22. Effect of endothelial p53 deletion on accumulation of collagen after TAC 66

Figure 5.23. Effect of endothelial p53 deletion on cardiac fibrosis

Figure 5.24. Effect of endothelial p53 deletion on markers of EndMT and myofibroblast transdifferentiation. 68

Figure 5.25. Analysis of endothelial and mesenchymal marker expression in mouse hearts after TAC.

Figure 5.26. Effect of p53 activation or inhibition on TGF $\beta$-induced endothelial-tomesenchymal transition of human cardiac microvascular endothelial cells....

Figure 5.27. Effect of endothelial p53 deletion on markers of EndMT and myofibroblast transdifferentiation.

Figure 6.1. HIF-1 and/or p53 regulated genes mediating adaptation to cellular stresses through activation of different pathways. 


\section{Abbreviations}

\begin{tabular}{|c|c|}
\hline$\alpha-\mathrm{SMA}$ & A-Smooth Muscle Actin \\
\hline$\beta-\mathrm{ME}$ & B-Mercaptoethanol \\
\hline$\beta-\mathrm{MHC}$ & B-isoform of myosin heavy chain \\
\hline ANP & Atrial natriuretic peptide \\
\hline AWTh & Anterior wall thickness \\
\hline $\mathrm{BNP}$ & Brain natriuretic peptide \\
\hline BSA & Bovine serum albumin \\
\hline cDNA & Complementary Deoxyribonucleic Acid \\
\hline CTGF & Connective tissue growth factor \\
\hline DAPI & Diamidino-2-Phenylindole dihydrochloride \\
\hline $\mathrm{ddH} 2 \mathrm{O}$ & Double distilled water \\
\hline DEPC & Diethylpyrocarbonate \\
\hline DMEM & Dulbecco's modified eagle medium \\
\hline DMSO & Dimethyl sulfoxide \\
\hline $\mathrm{dNTP}$ & Deoxynucleotide Triphosphate \\
\hline DPBS & Dulbecco's phosphate buffered saline \\
\hline $\mathrm{EC}$ & Endothelial cell \\
\hline ECBM & Endothelial cell basal medium \\
\hline ECGS/H & Endothelial cell growth supplement/heparin \\
\hline ECM & Extracellular matrix \\
\hline EDD & End-diastolic diameter \\
\hline EDTA & Ethylene diamine tetraacetic acid \\
\hline EGF & Epidermal growth factor \\
\hline EMT & Epithelial-mesenchymal transition \\
\hline End.MT & Endothelial-mesenchymal transition \\
\hline ESD & End-systolic diameter \\
\hline FACS & Fluorescence activated cell sorting \\
\hline FBS & Fetal bovine serum \\
\hline FCS & Fetal calf serum \\
\hline FITC & Fluorescein isothiocyanate \\
\hline
\end{tabular}

GAPDH Glyceraldehyde-3-phosphate dehydrogenase 
gDNA

HBSS

$\mathrm{HCl}$

HIF

IGF

$\mathrm{IgG}$

M199

$\mathrm{MgCl} 2$

$\mathrm{NaCl}$

$\mathrm{NaOH}$

$\mathrm{P} / \mathrm{S}$

PCR

PECAM-1

PFA

$\mathrm{pH}$

PWTh

RT

RT-PCR

SERCA2

SMC

$\mathrm{T} / \mathrm{E}$

TAC

TAE

Taq

TBS

TGF- $\beta$

Tie-2

TNF- $\alpha$

Tris

TSP

VEGF

VEGFR
Genomic DNA

Hank's balanced salt solution

Hydrochloric acid

Hypoxia-inducible factor

Insulin-like growth factor

Immunoglobulin G

Medium 199

Magnesium chloride

Sodium chloride

Sodium hydroxide

Penicillin/streptomycin

Polymerase chain reaction

Platelet endothelial cell adhesion molecule-1

Paraformaldehyde

Prepondirance of Hydrogen ions

Posterior wall thickness

Room temperature

Reverse transcription polymerase chain reaction

Sarcoplasmic / endoplasmic reticulum calcium atpase

Smooth muscle cells

Trypsin/EDTA

Transverse aortic constriction

Tris-acetate-EDTA

Thermus aquaticus

Tris-buffered saline

Transforming growth factor beta

Tyrosine kinase with immunoglobulin-like and

Tumor necrosis factor-alpha

Tris-(hydroxymethyl)-aminomethane

Thrombospondin

Vascular endothelial growth factor

Vascular endothelial growth factor receptor 


\section{Units}

\begin{tabular}{|c|c|}
\hline$\%$ & Percentage \\
\hline$\mu \mathrm{g}$ & Microgram \\
\hline$\mu 1$ & Microliter \\
\hline bp & Base pair \\
\hline $\mathrm{Ct}$ & Cycle threshold \\
\hline g & Gram \\
\hline hrs & Hours \\
\hline 1 & Liter \\
\hline M & Molar \\
\hline $\mathrm{mg}$ & Milligram \\
\hline $\mathrm{MHz}$ & Megahertz \\
\hline $\min$ & Minute \\
\hline $\mathrm{ml}$ & Milliliter \\
\hline $\mathrm{mM}$ & Millimolar \\
\hline$\mu \mathrm{M}$ & Micromolar \\
\hline $\mathrm{mm}$ & Millimeter \\
\hline ng & Nanogram \\
\hline $\mathrm{nm}$ & Nano meter \\
\hline${ }^{\circ} \mathrm{C}$ & Degree celsius \\
\hline OD & Optical density \\
\hline $\mathrm{rpm}$ & Rotations per minute \\
\hline $\mathrm{U}$ & Unit \\
\hline $\mathrm{w} / \mathrm{v}$ & Weight pro volume \\
\hline
\end{tabular}




\section{Summary}

Background- Accumulation of transformation related protein 53 (p53), apoptotic cell death and rarefication of myocardial angiogenesis may be involved in the development of cardiac dysfunction during chronic pressure overload.

Objective- The aim of this study was to determine whether prevention of endothelial cell apoptosis deletion of p53 in endothelial cells improves cardiac remodeling during pressure overload and prevents the transition from hypertrophy to heart failure.

Methods and Results - Mice with endothelial deletion of p53 (End.p53-KO) were generated by crossing $\mathrm{p} 53_{\mathrm{fl} / \mathrm{fl}}$ mice with mice expressing Cre recombinase under control of an inducible Tie2 promoter. Cardiac hypertrophy was induced by transverse aortic constriction. Serial echocardiography measurements revealed improved cardiac function and survival in End.p53-KO mice. In End.p53-WT controls, cardiac hypertrophy was associated with increased p53 levels, whereas banded hearts of End.p53-KO mice exhibited lower apoptotic cell numbers, both in endothelial and non-endothelial cells. Moreover, a higher cardiac capillary density, improved perfusion and elevated Hifl $\alpha$ and Vegf protein levels were observed, and inhibition or deletion of p53 also promoted endothelial sprouting in vitro and new vessel formation following hindlimb ischemia in vivo. Hearts of End.p53-KO mice exhibited markedly less interstitial fibrosis compared to End.p53-WT controls, and lower mRNA levels of p53-regulated genes involved in extracellular matrix production and turnover, or transcription factors involved in endothelial-to-mesenchymal transition.

Conclusions - Our data suggest that accumulation of p53 in endothelial cells contributes to cardiac apoptosis, blood vessel rarefication and fibrosis during chronic pressure overload and support the essential role of endothelial cells and cardiac angiogenesis for preserving contractile function during hypertrophy. 


\section{Introduction}

\subsection{Cardiac Hypertrophy and Heart Failure}

The term "hypertrophy" is derived from the Greek word hyper (increased growth) and trophe (diet or nutrition). ${ }^{1}$ Cardiac hypertrophy $(\mathrm{CH})$ is the thickening of the heart muscle (myocardium), which may result in the decrease in size of the left and right ventricle, but later also is associated with chamber dilation and cardiac enlargement. Heart failure (HF) is the incapability of the heart to sufficiently pump blood in response to the systemic needs. HF may be induced by a number of common disease stimuli, including long-standing hypertension, aortic stenosis, or familial hypertrophic and dilated cardiomyopathy, but also myocardial infarction associated with coronary artery disease. ${ }^{2}$ Cardiac hypertrophy and failure occur as a result of a combination of genetic, physiologic and environmental factors. ${ }^{3}$ In the absence of external stimuli such as increased afterload or a partial loss of tissue due to infarction, the myocardial size and function in the adult heart remain stable. When such conditions arise, the heart undergoes chamber enlargement and myocyte hypertrophy to adapt to the increased hemodynamic demands. ${ }^{4}$ This adaptive response of the heart to chronically increased afterload may fail if continued over the long term, resulting in the development of tissue ischemia, ventricular dilation and cardiac dysfunction. Like Neil Young said: "The same thing that makes you live can kill you in the end." ${ }^{5}$

\subsubsection{Morphologic Classification of Cardiac Hypertrophy}

Three stages of hypertrophic transformation of the heart were defined in the 1960s by Meerson and colleagues: ${ }^{6}$ (1) developing hypertrophy, which is excessive output load, (2) compensatory hypertrophy, in which the workload/mass ratio is normalized and resting cardiac output is maintained, and (3) heart failure with ventricular dilation/ dysfunction. Characteristically, two different types of hypertrophic phenotypes can be distingued, as shown in Figure 1.1: Physiological hypertrophy and pathological hypertrophy. Pathological hypertrophy is again divided into two types: a) concentric hypertrophy or compensatory hypertrophy due to pressure overload, which is characterized by parallel addition of sarcomeres and lateral growth of individual cardiomyocytes, and b) eccentric hypertrophy due to volume overload, which is characterized by addition of sarcomeres in 
series and longitudinal cell growth. ${ }^{1 ; 7}$ Physiological and pathological hypertrophy differ both at the morphological and the molecular level. ${ }^{8 ; 9}$

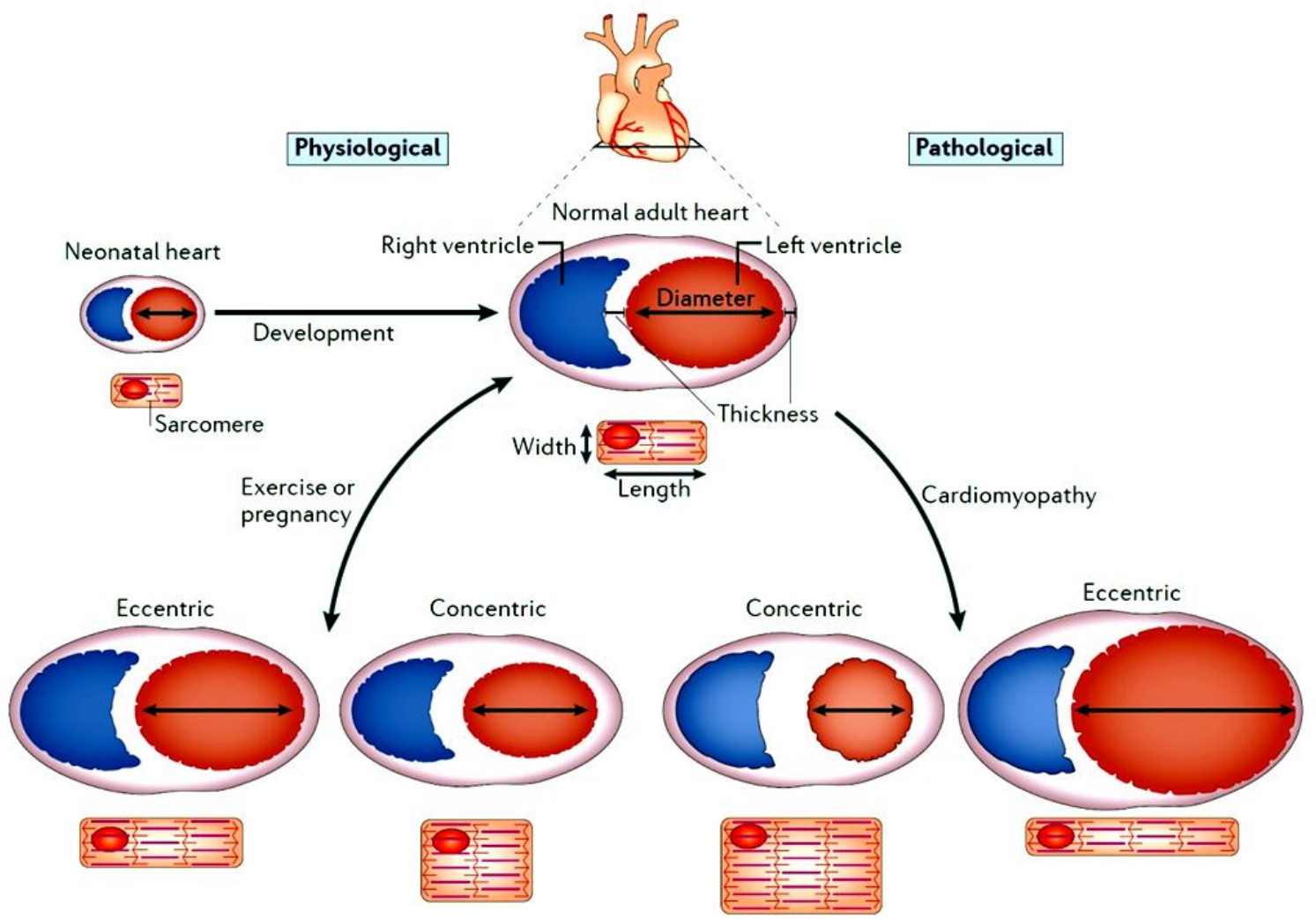

(Figure and part of legend adapted from Maillet $\mathrm{M}$ et al. Nature Reviews

Molecular Cell Biology 2013;14: 38-48)

Figure 1.1. The heart has the ability to increase its size and, depending on the stimulus, this results in physiological or pathological hypertrophy. Hypertrophy decreases ventricular wall stress by increasing the thickness of the wall. It follows Laplace's law, which says wall stress (or tension) is an inverse function of wall thickness $($ tension $=($ pressure $\times$ radius $) /(2 \times$ wall thickness $))$. Cardiac hypertrophy can be either eccentric or concentric growth based on the geometries of the heart, and individual non-pathological eccentric hypertrophy is characterized by an increase in ventricular volume with a coordinated growth in wall and septal thicknesses, where individual cardiomyocytes grow in both length and width. However, eccentric hypertrophy under pathological conditions (myocardial infarction or dilated cardiomyopathy) can lead to wall dilation with preferential lengthening of cardiomyocytes. Physiological stimulation can also induce a less pronounced form of eccentric hypertrophy. Concentric hypertrophy is characterized by a reduction in left ventricular chamber dimension and an increase in free wall and septal thicknesses, and individual cardiomyocytes typically increase in thickness more than in length (resulting in a decreased length/width ratio). Concentric hypertrophy usually arises owing to pathological conditions such as chronic hypertension or valvular stenosis. Isometric exercise training, such as wrestling or weight-lifting, also induces a milder form of concentric cardiac hypertrophy that is not known to be pathological. The heart can go from a normal state to a state of physiological hypertrophy and back, although pathological hypertrophy that produces heart failure may be less reversible. 


\subsubsection{Physiological Hypertrophy}

Physiological hypertrophy is an adaptive response to intermittent cardiac pressure and volume overload due to chronic exercise training, such as long distance running or swimming, or during pregnancy. In athletes and physically active persons, cardiac hypertrophy generally develops as an adaptive response to a physiological process which does not cause or lead to heart failure. However, in $20 \%$ of deconditioned athletes, ventricular dilation does not completely reverse to normal even after 5 years suggesting that permanent myocardial damage can occur as a consequence of prolonged training. ${ }^{10}$

The best example of physiological hypertrophy is postprandial cardiac hypertrophy in Burmese pythons, in which oxygen consumption increased sevenfold and ventricular mass increased significantly by $40 \%$ while digesting rats equal to $25 \%$ of body mass after $48 \mathrm{hrs}$ of fasting. This increase was fully reversible, and the ventricular mass returned to its fasting mass in post-digestion animals after 28 hrs. ${ }^{11 ; 12}$ Few reports showed that humans also undergo postprandial cardiac hypertrophy. Also during pregnancy, cardiac output increases to match placental blood flow, resulting in a physiological hypertrophy due to continuous volume overload. The heart undergoes modest eccentric cardiac hypertrophy and recovers fully to normal size of the heart after the delivery. ${ }^{13 ; 14}$ In few cases, some of patients after pregnancy develop peripartum cardiomyopathy. ${ }^{15}$

\subsubsection{Pathological Hypertrophy}

Pathological hypertrophy develops in response to mechanical stress induced by either extrinsic factors, such as increased pressure or volume overload in hypertension and valvular diseases, or intrinsic factors, such as ischemia-induced cardiac remodeling, which leads to contractile dysfunction and heart failure. ${ }^{1 ; 7}$ In contrast to physiological hypertrophy, pathological hypertrophy is associated with a loss of myocytes, fibrotic replacement (cardiac fibrosis) and cardiac dysfunction, and an increased risk of heart failure and sudden death.

During the initial stages of hypertrophy, the heart undergoes compensated growth to maintain cardiovascular function by cardiac myocyte enlargement and normalizing ventricle wall stress by formation of new sarcomeres. At later stages, the function of the hypertrophied heart 
ultimately decompensates leading to left ventricle dilation and heart failure (figure 1.2). The causes underlying this final decompensation are not completely understood.

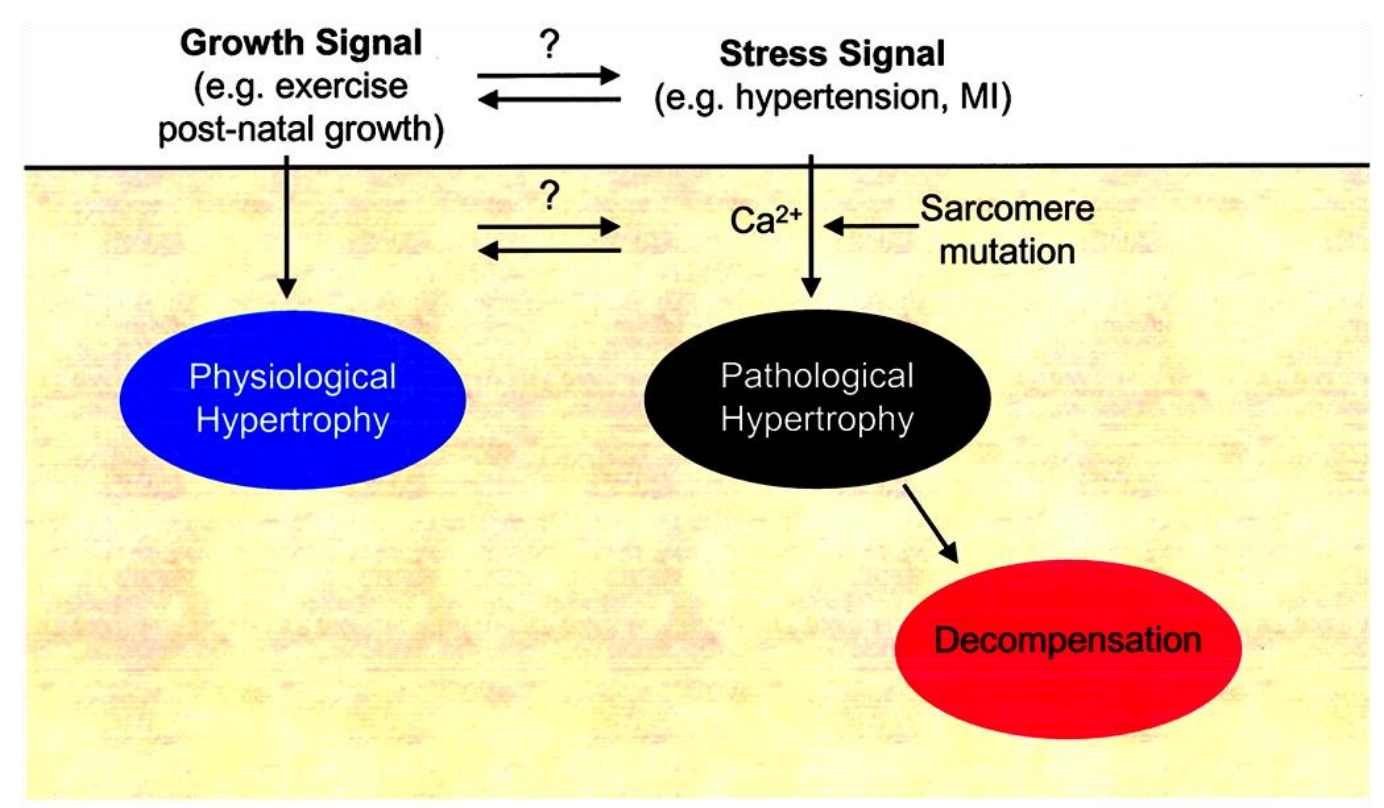

adaptive

maladaptive

(Picture and legend adapted from Frey N et al. Circulation 2004;109:1580-1589)

Figure 1.2. Physiological Versus Pathological Hypertrophy: Physiological hypertrophy is an adaptive response to growth signals. Pathological hypertrophy develops in response to stress signals. It is not known whether stress signals are unique or whether overstimulation ("too much of a good thing") evokes a pathological response. Similarly, it is not known whether physiological versus pathological hypertrophy derives from activation of unique "beneficial" signaling cascades or whether extreme activation of these same pathways evokes a pathological response. MI indicates myocardial infarction.

\subsubsection{Cardiac Hypertrophy Marker}

At the molecular level, cardiac hypertrophy is associated with increased expression of fetal genes and classical hypertrophic markers such as ANP, BNP, the $\beta$-isoform of myosin heavy chain $(\beta-\mathrm{MHC})^{16}$, or the $\alpha$-skeletal muscle isoform of actin $(\alpha \mathrm{SA}){ }^{7}$ In addition, several studies on cardiac hypertrophy or heart failure have shown decreased expression of the calcium cycling protein SERCA2a. ${ }^{17}$ In general, ANP and BNP mRNA levels tend to be increased in "ailing" hearts, i.e. those with reduced ventricular ejection or in heart failure with or without hypertrophy. Similarly, increased $\alpha$ SA and decreased SERCA mRNA levels 
are observed in hypertrophy models that progress towards heart failure. In contrast, $\beta$-MHC has been increased in several hypertrophy models with normal cardiac and cardiomyocyte function, indicating that it may not represent a maladaptive response. ${ }^{1}$

Recent studies have used microarrays to perform global, unbiased comparisons of regulated genes in physiological and pathological hypertrophy. Kong et al. ${ }^{18}$ compared transcript profiles in control rats (inactive Dahl salt-sensitive rats (Dahl/SS rat) on low-salt diet), physiologically hypertrophied rats (every day exercise), and compensated pathological hypertrophy (Dahl/SS rat on high-salt diet) using Affymetrix Rat Genome microarrays. Prolonged high-salt diet resulted in progression to heart failure, providing a fourth group (decompensated pathological hypertrophy) for analysis. ${ }^{18}$

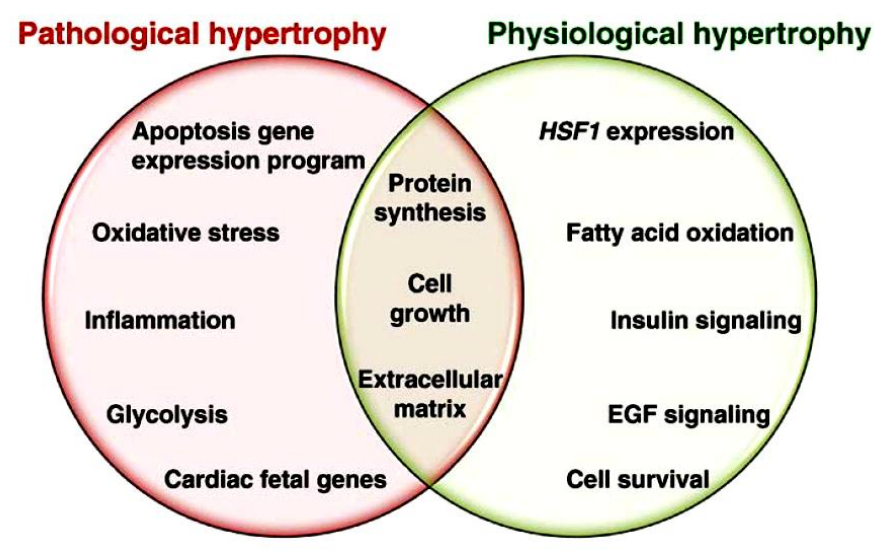

(Figure adapted from Bernardo B C et al. Pharmacology \& Therapeutics 2010;128:191-227)

Figure 1.3. Venn diagram depicting shared and distinct gene cluster expression profiles of physiological and pathological hypertrophy. HSF1 is a heat shock protein transcription factor that has recently been shown to be involved in physiological hypertrophy ${ }^{12 ; 18-20}$

In physiological hypertrophy, the genes largely regulated were representing metabolism and cellular growth, including members of the IGF/epidermal growth factor signaling pathway. In contrast, genes identified with pathological hypertrophy were largely from inflammation and stress-response clusters, and the genes expressed in decompensated hypertrophy/heart failure included apoptotic factors as shown in figure 1.3.

In addition to the above microarray data, clinical as well as experimental evidence suggests that the rarefication of cardiac capillaries promotes tissue hypoxia, cell death and replacement 
fibrosis and contributes to the progression from compensated hypertrophy to contractile dysfunction and heart failure. ${ }^{21-24}$

Angiogenesis plays an important role during the progression of adaptive cardiac hypertrophy. Inhibition of angiogenesis leads to decreased capillary density, which can hinder the cardiac growth and ultimately affect the adaptive hypertrophy to contractile dysfunction ${ }^{24}$. Therefore, angiogenesis plays a vital role in coordinating tissue growth with enough blood vessels to maintain cardiac function, and disruption of this coordination between angiogenesis and tissue growth in the heart may promote the transition from adaptive cardiac hypertrophy to heart failure. ${ }^{24}$ Induction of angiogenesis can also increase the cardiac mass without an external stimulus and cardiac function in Myocardial Infarction (MI) condition. ${ }^{25}$ Induction of angiogenesis by coexpressing VEGF and angiopoietin-1 has been shown to improve cardiomyocyte proliferation and reduction of cell apoptosis. ${ }^{26-30}$

\subsection{New Vessel Formation}

Endothelial cells receive multiple signals from their environment that eventually stimulates them to form new vessels. The development of new vessels can be considered in several different contexts. The two major processes responsible for the development of new blood vessels are "Vasculogenesis" and "Angiogenesis".

Vasculogenesis is the formation of blood vessels from endothelial progenitors or angioblasts in situ. ${ }^{31}$ This is the mechanism by which the primitive vascular plexus is formed. ${ }^{32}$ Vasculogenesis occurs in various steps starting with the generation of angioblasts from mesoderm; second is the assembly of angioblasts into vascular structures; third is the formation of a vascular lumen and finally, organization of continuous vascular networks. Blood vessels formed by the process of vasculogenesis are immature, that means they are not accompanied by pericytes, smooth muscle cells (SMCs) and other associated cells. ${ }^{33}$

Angiogenesis is the progression of vascular growth by sprouting, bridging, and extension of capillaries from pre-existing blood vessels. ${ }^{34-37}$ In contrast to vasculogenesis, angiogenesis involves vascular growth and maturation. The angiogenesis process starts with the proteolytic degradation of interstitial tissue and the basal membrane of the parental vessel and 
endothelial cells migrate, proliferate, avoid apoptosis and finally differentiate into new vascular tubes in response to angiogenic stimuli from their environment. ${ }^{35 ; 38 ; 39}$

Most of the normal tissues in the adult have a low rate of cell turnover, with an exception of the epithelial tissues and the hematopoietic elements in the bone marrow. Smooth muscle and endothelial cells of the blood vessel have a turnover time of many months. Only $0.01 \%$ of all endothelial cells of a normal adult are dividing at any given moment. However, in response to angiogenic stimulation, endothelial cells enter into an actively proliferating state to form new blood vessels. ${ }^{40}$ The different stages of angiogenesis are shown in figure 1.4.

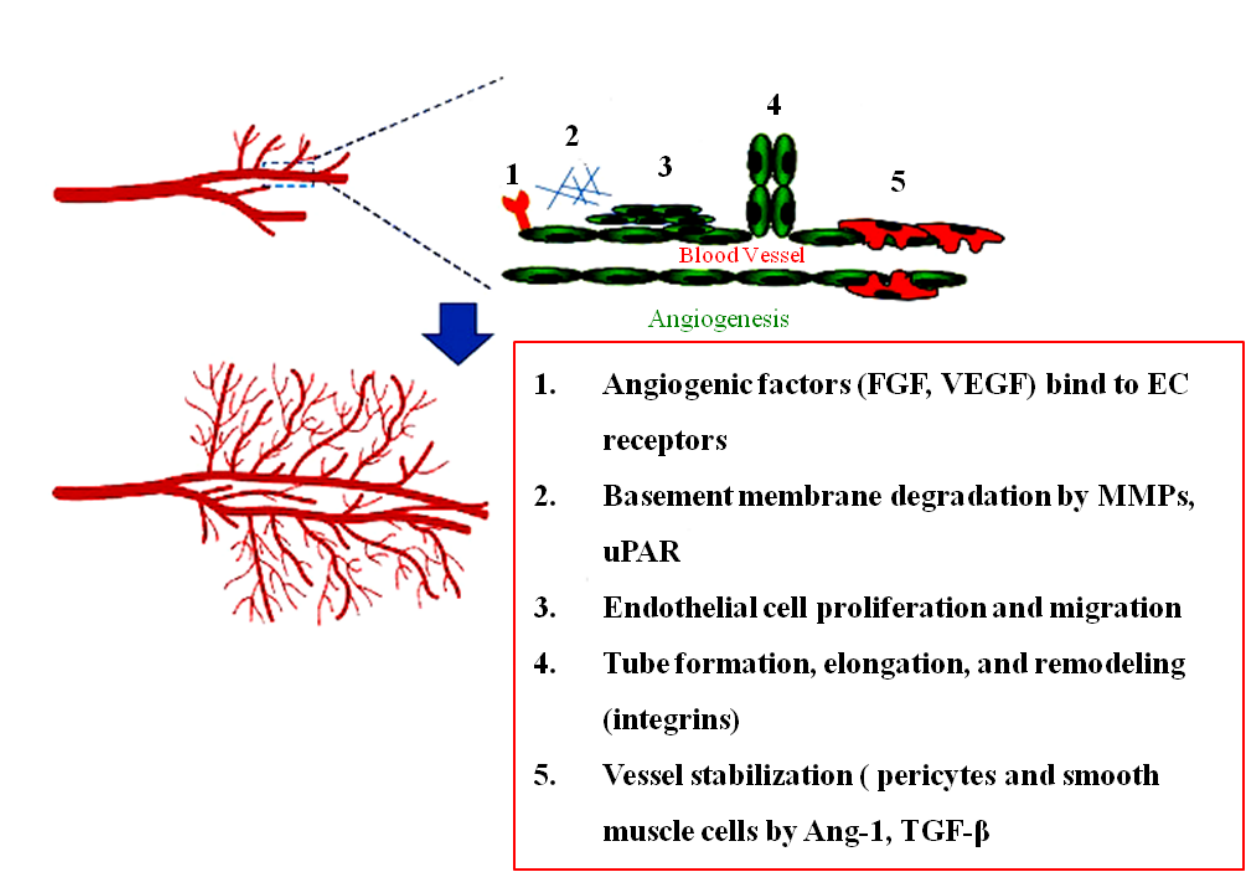

(Figure and legend modified from: Yoo SY et al. Mediators Inflamm 2013;2013:127170)

Figure 1.4. Processes in angiogenesis. (1) Angiogenic factors bind to their receptors on endothelial cells and activate signal transduction pathways. (2) Degradation of the extracellular matrix. (3) Endothelial cells migrate out of the preexisting capillary wall and proliferate. (4) Integrins are expressed by endothelial cells, thus facilitating their adhesion to the extracellular matrix and their migration for tube formation. (5) Angiopoietin-1 binds to Tie-2 receptors and stimulates pericyte recruitment and vessel stabilization.

Extracellular signals involved in these processes are mainly secreted factors that act in a paracrine fashion. Specialized receptors like G-protein-coupled receptors, tyrosine kinase receptors, tyrosine-kinase associated receptors and serine-threonine kinase receptors are the main transmembrane receptors that transduce angiogenic signals. The pathways of signal 
transduction between the surface receptors and the final effectors of the modified endothelial cell behavior are partly known.

\subsubsection{Vascular Endothelial Growth Factors and Receptors}

Vascular endothelial growth factor-A (also called VEGF) is the master regulator of angiogenesis and vascular permeability, as it is required to initiate the formation of immature vessels by vasculogenesis or angiogenic sprouting. ${ }^{41-45}$

After the discovery of VEGF-A, 4 other members in the human VEGF family have been identified: VEGF-B, VEGF-C (also called VEGF-2), VEGF-D, placental growth factor (PlGF). Recently an entirely unrelated family of growth factors known as the angiopoietins (Ang) and particular members of the very large ephrin family have been identified as having unique effects on the endothelium. ${ }^{27 ; 46 ; 47}$ As said earlier, VEGF is required to initiate the formation of immature vessels by vasculogenesis or angiogenic sprouting. Ang1 and ephrinB2 are required for further remodeling and maturation of this immature vasculature, particularly as endothelial cells integrate with supporting cells such as smooth muscle cells and pericytes. Following vessel maturation, Ang1 continues to be important for maintaining the quiescence and stability of the mature vasculature. ${ }^{48}$ The downstream signals of VEGFs in the vascular endothelium are mediated by 3 tyrosine kinase signaling receptors (VEGF receptor [VEGFR]-1, -2, and -3) as shown in Figure 1.5. 

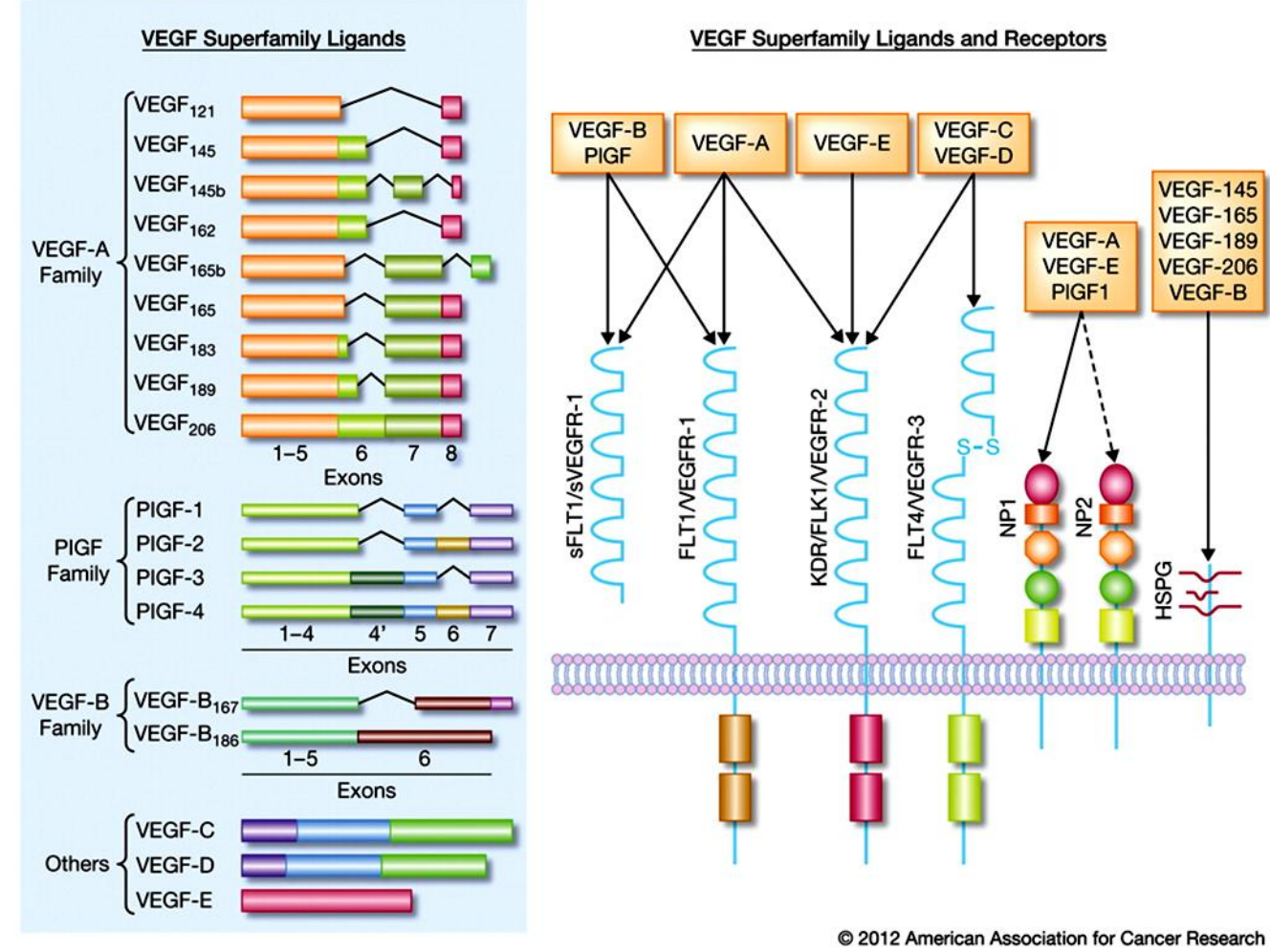

(Figure adapted from Rahimi N. Mol Cancer Ther 2012;11:538-48)

Figure 1.5. VEGF super family ligands and receptors. A schematic of VEGF ligands and their interactions with VEGF receptors.

Hypoxia-inducible factors (HIFs) are oxygen sensitive transcription factors, which regulate the expression of hypoxia-inducible genes (e.g. VEGF) at lower oxygen concentrations. Stabilization of HIF1 $\alpha$ activates the transcription of genes that participate in key mechanisms involved in enhancing tissue perfusion and oxygenation, such as angiogenesis, cell differentiation, anaerobic metabolism, and apoptosis. ${ }^{49}$ HIF1 $\alpha$ plays an important role in vascular development by increasing VEGF-A expression in acute ischemic conditions. ${ }^{50 ; 51}$

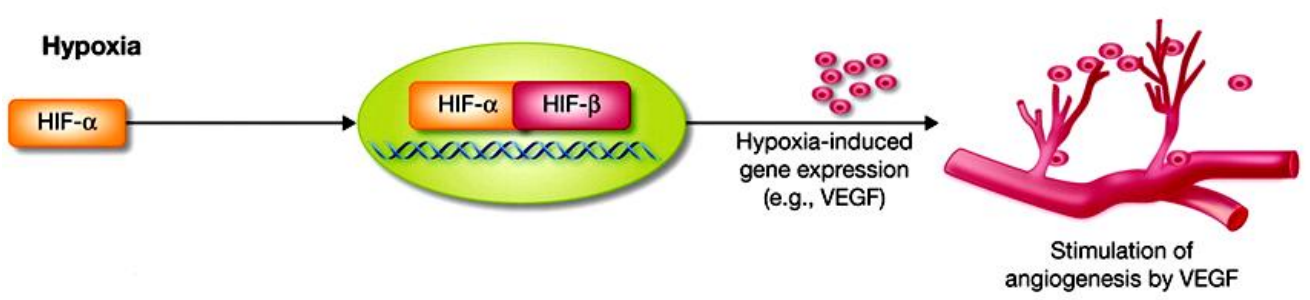

(Figure adapted and modified from Rahimi N. Mol Cancer Ther 2012;11:538-48)

Figure 1.6. Role of HIF1 $\alpha$ in the expression of VEGF and the stimulation of angiogenesis 


\subsubsection{Angiogenesis Stimulators and Inhibitors}

Angiogenesis is considered to depend on the balance between endogenous stimulators and inhibitors. ${ }^{52}$ Recent studies suggests that endothelial cell apoptosis plays an important role to maintain or fix damaged blood vessels during angiogenesis. ${ }^{53}$ There are several enhancers/ stimulators of endothelial survival including VEGF, ${ }^{28 ; 54 ; 55}$ basic Fibroblast Growth Factor (bFGF), ${ }^{56}$ integrins (e.g. $\left.\alpha v \beta 3\right),{ }^{57-60}$ Nitric Oxide (NO), ${ }^{61}$ platelet-derived growth factor, HIF$1 \alpha$, as well as angiogenic oncogenes, such as Ras, and tumor suppressors, such as p53. ${ }^{62}$ Inhibitors or inducers of endothelial cell apoptosis are Thrombospondin-1 (TSP-1), ${ }^{63-65}$ Angiostatin, ${ }^{66}$ Tumor Necrosis Factor (TNF), ${ }^{67}$ Transforming Growth Factor- $\beta$ (TGF- $\beta$ ), ${ }^{68}$ and many more. Endogenous inhibitors of angiogenesis include various antiangiogenic peptides, hormone metabolites, and apoptosis modulators. Some of the above listed inhibitors and others were again divided into matrix and non- matrix derived inhibitors (in Figure 1.7). ${ }^{69}$

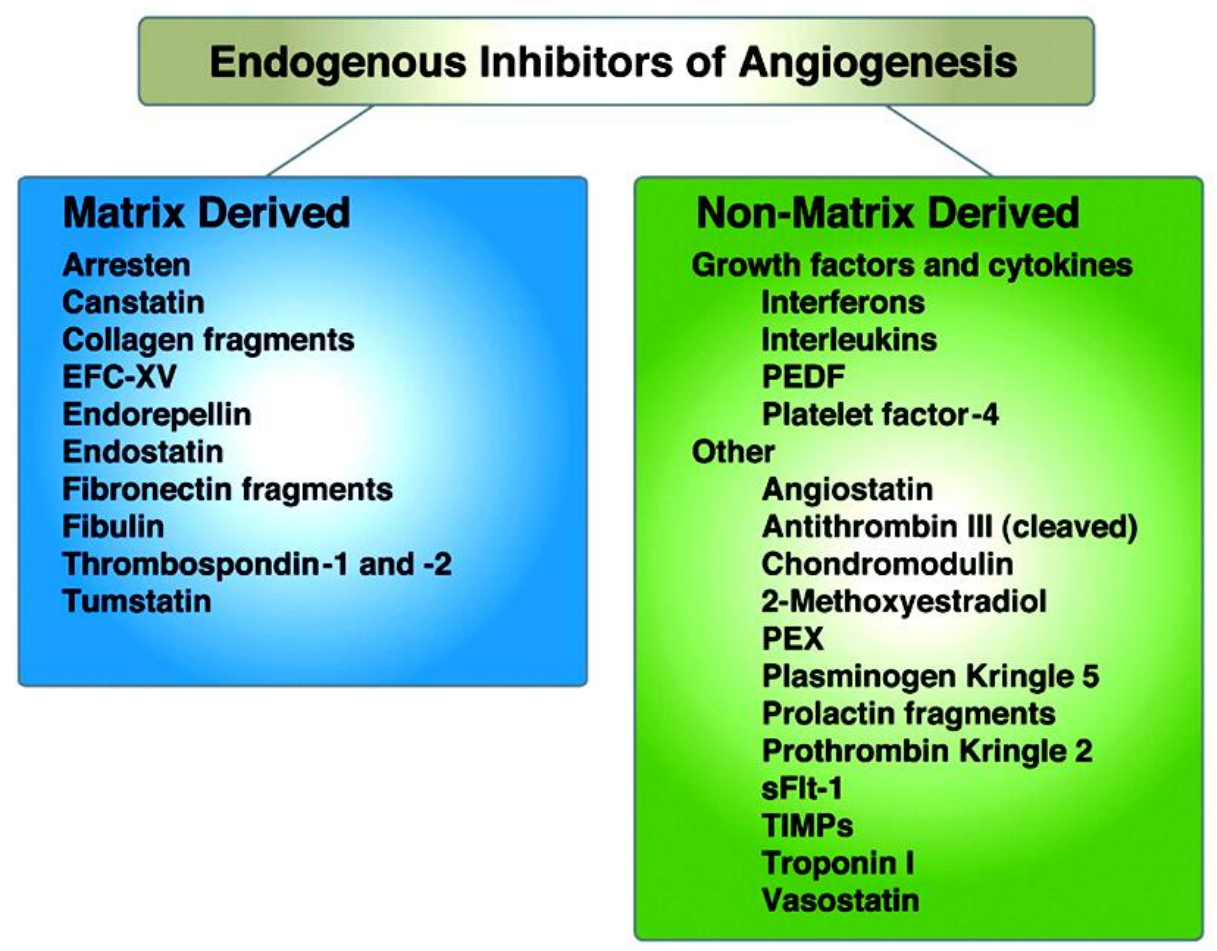

(Figure adapted from Nyberg P et al. Cancer Res 2005;65:3967-79.)

Figure 1.7. Endogenous angiogenesis inhibitors 
Cardiac hypertrophy initially promotes adaptive angiogenesis by HIF $1 \alpha$-dependent induction of pro-angiogenic factors. By this, the heart maintains its compensatory state during pressure overload but, in long term, can lead to hypertension. ${ }^{70}$ p53 is upregulated during hypertrophy and inhibits the protective pro-angiogenic activity of HIF $1 \alpha$, eventually leading to heart failure. A central role of cardiac endothelial cells and angiogenesis is supported by findings that inhibition of new blood vessel formation or inadequate angiogenesis during the development of cardiac hypertrophy is the main reason for that the adaptive cardiac hypertrophy transmits the development of left ventricular (LV) dysfunction and ultimately heart failure. ${ }^{71 ; 72}$ Whereas stimulation of angiogenesis by increasing the production and secretion of angiogenic growth factors, such as VEGF, in the hypertrophied heart improves cardiac function and delays the onset of heart failure. ${ }^{25 ; 29 ; 73}$ In recent years, a number of publications have revealed the anti-angiogenic properties of p53. ${ }^{74-79}$ However, the molecular mechanisms involved in the regulation of cardiac angiogenesis and in particular, the role of p53 in the reduction of cardiac vessel density during pathological hypertrophy are incompletely understood.

\subsection{Tumor Suppressor Protein p53 in Angiogenesis and Heart Failure}

p53 is a tumor suppressor protein with proapoptotic and antiproliferative activities. p53 transcriptionally activates variety of molecules or genes that induce apoptosis, cell growth arrest, inhibit angiogenesis, functions in DNA repair, regulate senescence and others (figure 1.8). p53 is upregulated in adaption to harmful stimuli, including hypoxia, oxidative stress, UV damage or infections. In the cardiovascular system, p53 plays a crucial role in development of heart failure.

For example, elevated levels of p53 were reported in myocardial biopsies of patients with heart disease ${ }^{80}$ and found to progressively increase with disease severity. ${ }^{81}$ Increased apoptotic cell numbers have also been described in rodents following transverse aortic constriction (TAC), ${ }^{82}$ a model frequently used to study the cellular and molecular changes during cardiac hypertrophy and failure. ${ }^{83}$ Puma (p53-upregulated modulator of apoptosis) inactivation may serve as a preferential target to prevent heart failure induced by cellular stress. ${ }^{84}$ Supporting a causal role of apoptotic cell death and p53 in the development of heart failure, experimental studies could show that global p53 deficiency protects against cardiac 
injury, ${ }^{85 ; 86}$ whereas p53 activation accelerates LV function deterioration. ${ }^{87}$ Disruption of p53 activation in adipose tissue attenuated inflammation and improved insulin resistance but also ameliorated cardiac dysfunction induced by chronic pressure overload. ${ }^{88}$ In contrast, recent studies showed that in radiation therapy, p53 functions in endothelial cells to protect mice from myocardial injury induced by whole heart irradiation. ${ }^{89}$ p53 induces connective tissue growth factor (CTGF) expression and promotes liver fibrosis, suggesting that the p53/CTGF pathway may be a therapeutic target in the treatment of liver fibrosis. ${ }^{90}$

In addition to its role as master regulator of cellular senescence, cell cycle arrest and apoptosis, p53 is involved in the transcriptional regulation of genes controlling diverse biological processes, such as differentiation, migration or angiogenesis. ${ }^{91}$ P53 plays as antiangiogenic by transcriptionally activating the $\alpha$ (II) collagen prolyl-4-hydroxylase gene, resulting in the extracellular release of antiangiogenic fragments of collagen type 4 and $18 .{ }^{92}$ However, the role of endothelial p53 expression for the cardiac response to chronic pressure overload is largely unknown.

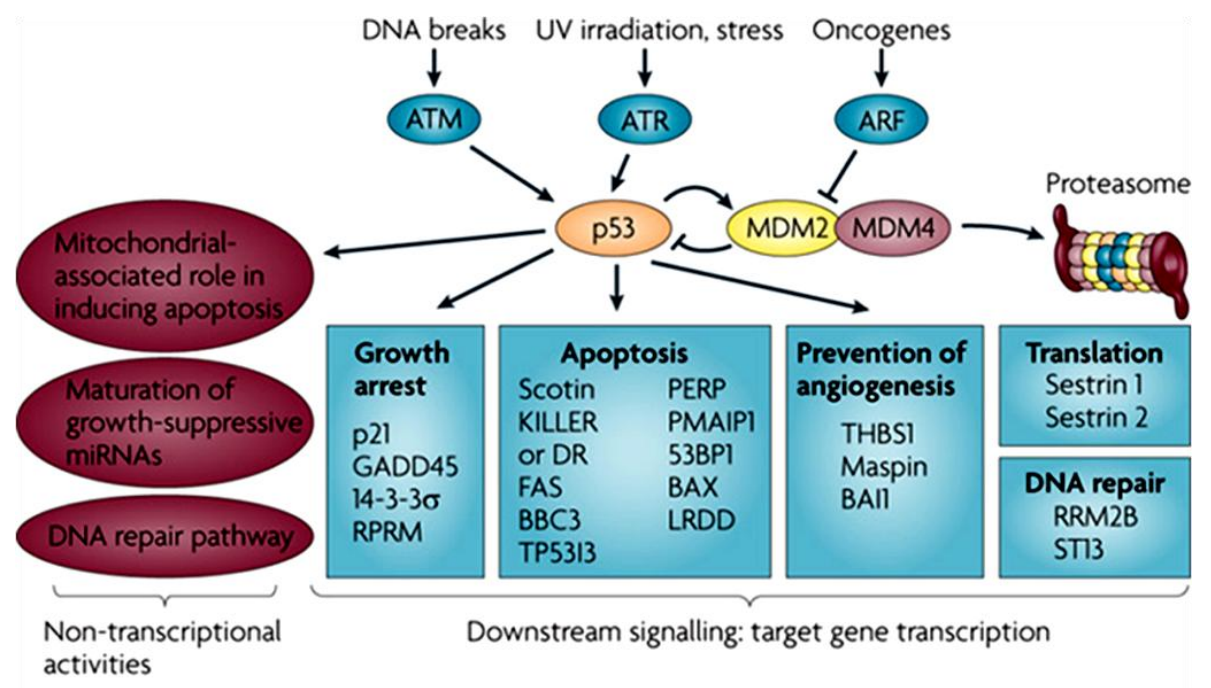

Nature Reviews | Cancer

(Figure adapted from Christopher J et al. Nature Reviews Cancer 2009; 9, 862-873)

Figure 1.8. The p53 pathway. $P 53$ is at the centre of a complex web of biological interactions that translates stress signals into cell cycle arrest, apoptosis and prevention of angiogenesis. MDM2 and MDM4 proteins bind to p53 in non-stressed conditions and ubiquitylate p53 and target it for degradation by the proteasome. 


\subsection{Cardiac Fibrosis}

Cardiac fibrosis $(\mathrm{CF})$ is characterized by the accumulation of extracellular matrix (ECM) proteins in the cardiac interstitial spaces, and contributes to both systolic and diastolic dysfunction in many cardiac pathophysiological conditions. ${ }^{93} \mathrm{CF}$ is a major determinant of cardiac function and contractility. CF leads to distorted organ structure which is often observed in patients with advanced heart failure. ${ }^{94} \mathrm{CF}$ increases myocardial stiffness and induces electrical heterogeneity, leading to systolic and diastolic dysfunction and sudden death.

Cardiac fibroblasts are activated by a variety of pathological stimuli, thereby undergoing proliferation, differentiation to (myo-)fibroblasts, and production of various cytokines and ECM proteins within the heart. ${ }^{95 ; 96}$ The interactions of myocytes, ECM, and cardiac fibroblasts controls physiological cardiac function. ${ }^{97}$ Cardiac fibroblasts maintain ECM homeostasis to facilitate proper cardiac contraction. ${ }^{98}$ Fibroblasts regulate cardiomyocyte function by secreting various growth factors ${ }^{99}$ and via direct cell-cell interactions. ${ }^{100}$ They are crucial for angiogenesis and vascular homeostasis in the heart. ${ }^{101}$ Based on the morphological appearance fibroblasts are classified as inactive or active fibroblasts. The active fibroblasts appear to be large and produce more collagen leading to cardiac fibrosis. ${ }^{102}$ Fibrosis occurs in two forms, reactive interstitial fibrosis or replacement fibrosis. ${ }^{103 ; 104}$ Reactive interstitial fibrosis is observed in left ventricular pressure overloading models which progresses without loss of cardiomyocytes. This initial reactive interstitial fibrosis, which is an adaptive mechanism, will progress into replacement fibrosis, characterized by cardiomyocyte hypertrophy and necrosis. ${ }^{105}$

\subsubsection{The Origin of Cardiac Fibroblasts during Embryonic Development}

The actual number of fibroblasts in the cardiac tissue remains unknown. It has been known that the adult mouse heart contains approximately 55\% myocytes and only $45 \%$ non-myocyte cells ( $27 \%$ fibroblasts). But, the adult rat heart consists of $30 \%$ myocyte cells and $70 \%$ nonmyocyte cells ( $\sim 67 \%$ fibroblasts). ${ }^{104 ; 106 ; 107}$ Because of distinct origins, cardiac fibroblast is an extremely heterogenic cell population. Cardiac fibroblasts can be derived either from 1) epithelial-mesenchymal transition (EMT) ${ }^{108}$ and gradually differentiate into a fibroblast 
phenotype $^{109 ; 110}$ under the influence of growth factors, 2) resident fibroblasts, 3) cardiac endothelium via endothelial-mesenchymal transition (EndMT) or bone marrow-derived circulating progenitor cells, monocytes and fibrocytes (Figure.1.9). ${ }^{104}$

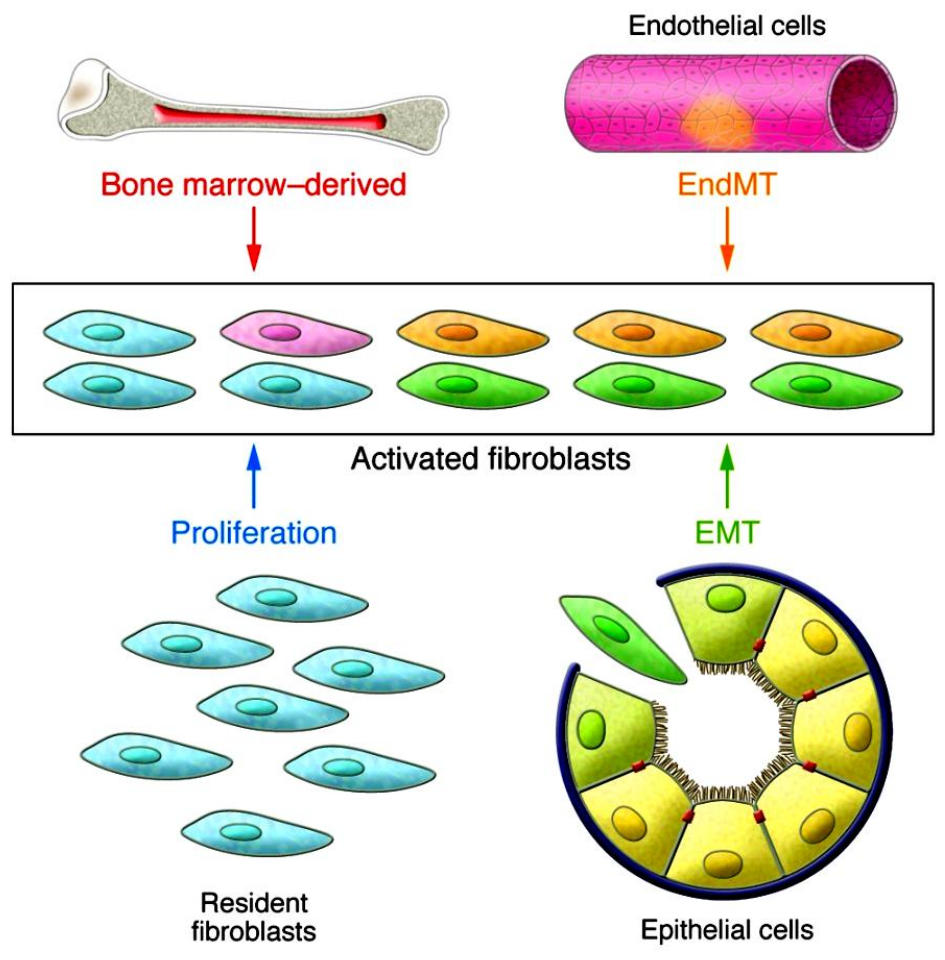

(Figure adapted from Kalluri R and Weinberg RA, J Clin Invest 2010;120:1786)

Figure 1.9. Origin and sources of fibroblasts during organ fibrosis. Four possible mechanisms are depicted. One study suggests that about $12 \%$ of fibroblasts are from bone marrow, about $30 \%$ can arise via local EMT involving tubular epithelial cells under inflammatory stress, and about 35\% are from EndMT, the remaining percentage likely emerge via proliferation of the resident fibroblasts and other still unidentified sources.

\subsubsection{Endothelial-to-Mesenchymal Transition (EndMT)}

EndMT is a complex biological process in which endothelial cells under the pressure of profibrotic stimuli (e.g. TGF $\beta$ and hypoxia) acquire a mesenchymal or myofibroblastic phenotype with the expression of mesenchymal cell products such as $\alpha$-SMA, vimentin or type I collagen while losing the characteristics of endothelial cells (figure 1.10). ${ }^{111}$

In cardiac fibrosis, about $30 \%$ of activated fibroblasts are generated from endothelial cells via a cellular phenotype switch which is referred to as endothelial-mesenchymal transition $(\text { EndMT })^{112}$. Upon phenotypic change these cells resemble as fibroblasts and leave the 
microvascular bed and enter the interstitium. Hence, EndMT contributes to cardiac fibrosis both by contributing to fibroblast accumulation and also to microvascular rarefication. ${ }^{112}$

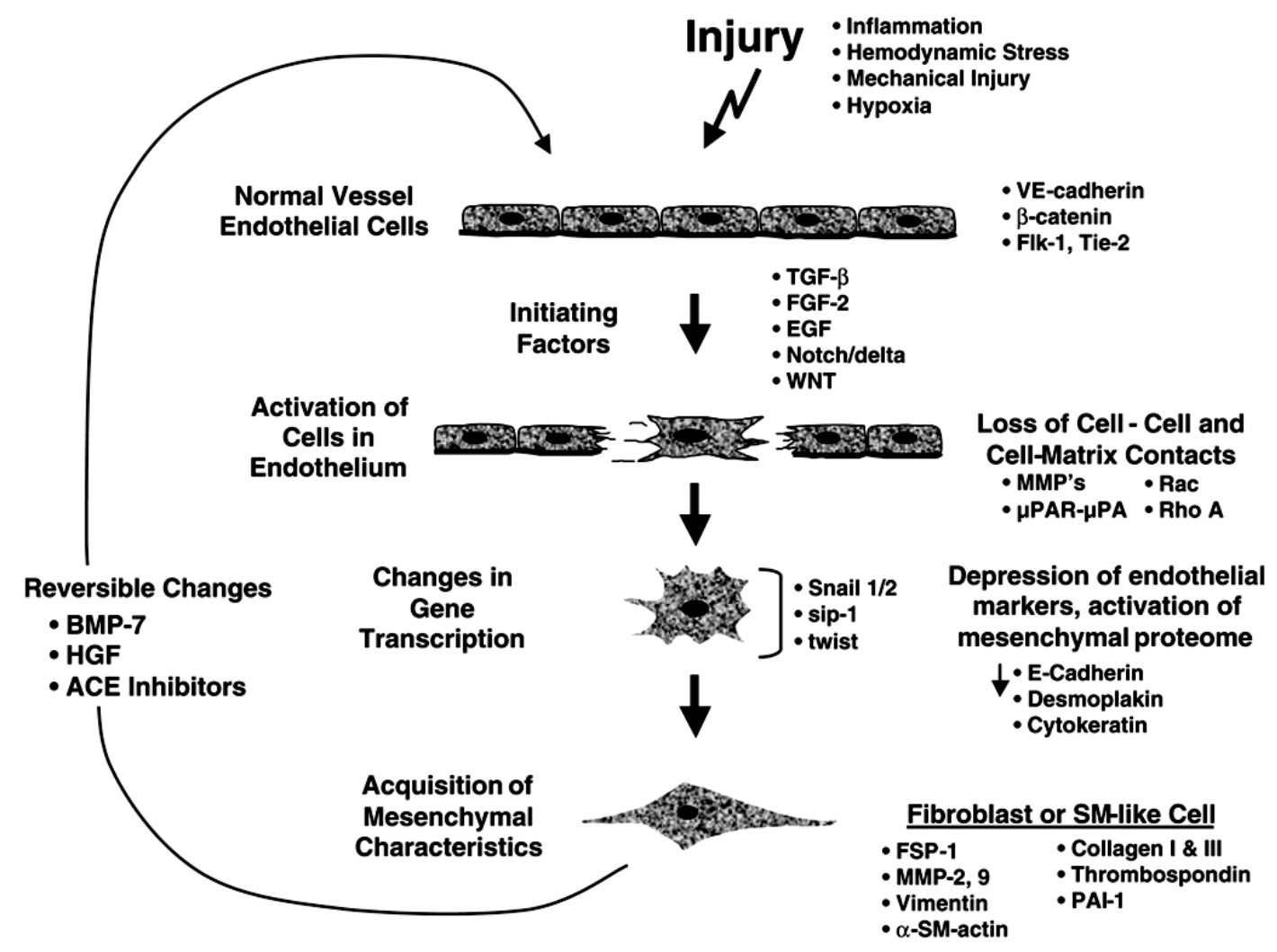

(Picture adapted from Arciniegas E et al. Am J Physiol Lung Cell Mol Physiol 2007; 293:L1-L8)

Figure 1.10. Schematic diagram illustrating potential mechanisms involved in endothelial-mesenchymal transition. Many initiating factors, which can lead to loss of cell-cell contacts, have been described. These factors are capable of activating metalloproteinase and serine protease family members and are important in loss of cell-cell contact. Interruption of cell-cell or cell-matrix contact, activates transcription factors, which are actively involved in suppression of E-cadherins and other endothelial-specific proteins. Upregulation of a mesenchymal gene program follows. Importantly, in some situations, this process may be reversible. HGF, hepatocyte growth factor; BMP, bone morphogenetic protein; TGF, transforming growth factor; MMPs, matrix metalloproteinases.

Recent in vivo evidence using an endothelial lineage tracing approach suggested that a substantial number of cardiac fibroblasts are derived from resident endothelial cells, which have undergone endothelial-to-mesenchymal transition (EndMT) in response to transforming growth factor (TGF)- $\beta .^{112 ; 113}$ Although an association between endothelial cell apoptosis and EndMT has been suggested, ${ }^{114}$ a causal role for p53 in this process remains to be shown. 


\subsection{Hypothesis and Study Objective}

The tumor suppressor protein p53 is proapoptotic in function, and a number of recent data have shown that p53 is upregulated during heart failure. In this study we hypothesized that in response to cellular stress, p53 undergoes stabilization and promotes apoptosis of endothelial cells, leading to decrease in capillary density in the heart, thereby increasing hypoxia and promoting to cell death and fibrosis. Hence, in this study, we would like to examine whether deletion of the p53 in endothelial cells is able to preserve the cardiac microvasculature, to prevent pathological cardiac remodeling and the development of fibrosis in response to chronic pressure overload and to postpone the transition from cardiac hypertrophy to heart failure. 


\section{Materials and Methods}

\subsection{Laboratory Equipment}

$0.22 \mu \mathrm{m}$ filter unit, Millex-GS Millipore (GLGS0250S)

ART-MICRA D-1, No 30072, homogeniser

Autoclave, Tuttnauer 5075 ELV, Biomedis

BANDELIN SONOPULS sonicator

Biosafety cabinet with aspirator for tissue culture, CA/RE5, W. Krannich

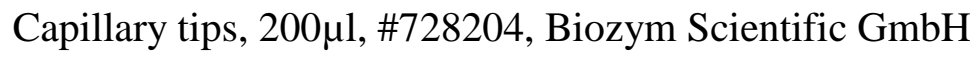

Chemical fume hood, Norddeutsche Laborbau

$\mathrm{CO}_{2}$ incubator, SANYO, W. Krannich

CODA $^{\mathrm{TM}}$ Monitor, Kent scientific corporation

DNA electrophoresis set, Bio-Rad

Drying oven, Heraeus

Eppendorf centrifuge 5415D

Eppendorf centrifuge 5810R

Eppendorf mastercycler gradient

FACS $5 \mathrm{ml}$ polystyrene round-bottom tube, 12 X $75 \mathrm{~mm}$, BD Falcon

FACSAria II cell sorter, BD Bioscience

FACSVantage SE system, BD Biosciences

Fluorescence microscope (AXIO ZEISS) coupled to a digital camera

Freezing container Nalgene Labware (5100-0001)

Gel photo printer, SEIKO Precision DVP-1200, Biozym

Glass Pasteur pipettes, $230 \mathrm{~mm}$

Injection needle 0.6 X $30 \mathrm{~mm}, 23 \mathrm{G}$ 1/4"-Nr.14

$\mathrm{iQ}^{\mathrm{TM}} 5$ Multicolor Real Time PCR Detection system, BIO-RAD

Labcycler, Sensoquest, Biotechnica

Laminar flow hood with a HEPA filter

Microscope coverglasses, 18 X18 mm, Gerhard Menzel GmbH

Microscope glass slides, Gerhard Menzel GmbH

Microtome RM2165, Leica

Microwave oven 
Mini Trans-Blot® Cell, Serial No. 37S/5986

Mini-PROTEAN Precast Gels

Mini-PROTEAN® 3 Cell, Serial No. 67S/12383

MiniVent, mouse ventillator, Type: 845, Hugo Sachs Electronics

MultiImage light cabinet, Alpha Innotech Corporaton

Multi-micropipette, Eppendorf AG

NanoDrop ${ }^{\circledR}$ ND-1000 Spectrophotometers, Thermo Scientific

PERIMED, Periscan PIM 3

Pipette tips for volumes $0.1-1000 \mu 1$

Pipettes for volumes 0.1-1000 $\mu 1$

Power Pac 200, BIO-RAD

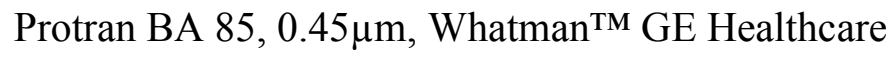

Reaction tubes (1.5 and $2 \mathrm{ml})$

Reaction tubes (15 and $50 \mathrm{ml}$ )

Slide storage boxes

Standard plastic pipettes $(2,5,10,25 \mathrm{ml})$

Syringe, Omnifix 40 Solo, $1 \mathrm{ml} / 40$ I.U. B.Braun (for matrigel injection)

Upright microscope for fluorescence or DIC microscopy, Olympus BX51

Vortex-Genie 2, Schuett Labortechnik

Water bath set at $37^{\circ} \mathrm{C}$

Water bath with accurate temperatures between 15 and $99^{\circ} \mathrm{C}$ 


\subsection{Chemicals and Reagents}

Product

0.5M EDTA, $\mathrm{pH} 8.0$

$1 \mathrm{M}$ Tris $\mathrm{pH} 7.0$

$1 \mathrm{M}$ Tris $\mathrm{pH} 8.0$

2-Mercaptoethanol

2-Propanol

2X Laemmli Sample Buffer

$5 \mathrm{M} \mathrm{NaCl}$

Acetic acid $96 \%$

Acetone

Antibody Diluent

Biozym LE Agarose

Chem Cruz, Blotto, non-fat dry milk

Diamidino-2-Phenylindoledihydrochloride

(DAPI)

Ethanol absolute for analysis

FITC-Griffonia (Bandeiraea)

Simplicifolia lectin I, Isolectin B4

FITC-Griffonia lectin I

Hydrochloric acid (HCl) $37 \%$

Ketamin $10 \%$

Mayer's hematoxylin

Methanol

Mounting medium

Paraformaldehyde (PFA)

PCR nucleotide mix

Pierce®BCA Protein Assay Reagent A

Pierce ${ }^{\circledR} B C A$ Protein Assay Reagent B

Ponceau S solution

Proteinase K, recombinant PCR Grade
Supplier

Cat. Number

Ambion

Ambion

AM9260G

Ambion

BIO-RAD

Sigma

BIO-RAD

Ambion

Carl Roth

Merck

Dako

AM9851

AM9856

161-0710

278475

161-0737

AM9760G

T179.2

8.222 .511 .000

S-3022

Biozym

840004

Santa Cruz

SC-2325

Sigma

D-9542

Merck

1.00983 .1011

Vector

FL-1201

Vector

FL-1101

Fluka/Sigma

84422

Medistar

13690.00.00

Sigma

MHS32

J.T.Baker

8402

Vector

H-1000

Sigma

P6148

Promega

C114H

Thermo Scientific

23228

Thermo Scientific

1859078

Sigma

P7170-1L

Roche

3115828001 
RNaseZap

Rotiphorese® Gel 30 (37,5:1)

SDS $20 \%$ Solution

Sodium Chloride $(\mathrm{NaCl}), 0.9 \%$

Sodium Hydroxide $(\mathrm{NaOH}), 2 \mathrm{~N}$

SuperSignal ${ }^{\circledR}$ West Pico

Chemiluminescent Substrate

Triton X-100

TWEEN $^{\circledR} 20$

Xylariem(Xylazine)

Xylol (Isomere)

\subsection{Kits}

\section{Product name}

FITC Annexin V Apoptosis

Detection Kit I

GoTaq® Flexi DNA Polymerase

In Situ Cell Death Detection Kit,

Fluorescein

Random Primers

RiboSafe RNase Inhibitor

SYBR $^{\circledR}$ Green PCR Master Mix
Ambion

AM9780

Roth

3029.1

Ambion

AM9820

Braun

6697366.00 .00

Merck

1.091 .361 .000

Thermo Scientific $\quad 34080$

Fluka/Sigma

93426

Sigma

P1379

Riemser

400177.00.00

Roth

9713.3
Company

BD Pharmingen ${ }^{\mathrm{TM}}$

556547

Promega

M8305

Roche

11684795910
Promega

Bioline

65027

Applied Biosystems

4309155 


\subsection{Buffers and Solutions}

10X PBS

10X TBS

10X Wet Transfer Buffer

$4 \%$ PFA

4X Separating Buffer

4X Stacking Buffer

50X TAE buffer

5X Electrode Running Buffer
$80 \mathrm{~g} \mathrm{NaCl}, 2 \mathrm{~g} \mathrm{KCl}, 14.4 \mathrm{~g} \mathrm{Na}_{2} \mathrm{HPO}_{4}$ and $2.4 \mathrm{~g} \mathrm{KH}_{2} \mathrm{PO}_{4}$

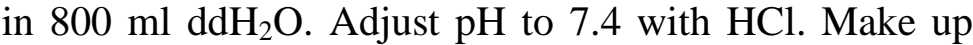
the final volume to 1 liter and sterilize by autoclave.

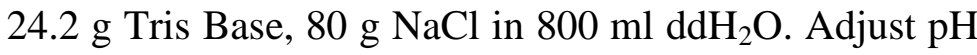
to 7.6 with $\mathrm{HCl}$. Make up the final volume to 1 liter.

$30.3 \mathrm{~g}$ Trizma base and $144 \mathrm{~g}$ glycine in 1 liter $\mathrm{ddH}_{2} \mathrm{O}$. For 1X Transfer buffer: combine $100 \mathrm{ml}$ 10X Transfer

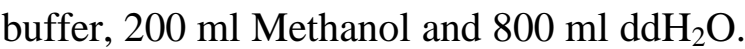

$4 \mathrm{~g}$ PFA in $100 \mathrm{ml}$ of $\mathrm{PBS}$ and heat at $60^{\circ} \mathrm{C}$ with a stir bar in a chemical hood until the solution became clear.

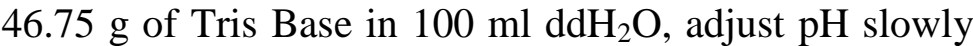
to 8.8 with concentrated $\mathrm{HCl}$, add $1 \mathrm{~g}$ SDS and dissolve thoroughly. Make up the final volume to $250 \mathrm{ml}$ with $\mathrm{ddH}_{2} \mathrm{O}$.

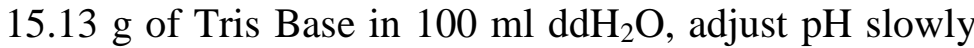
to 6.8 with concentrated $\mathrm{HCl}$, add $0.25 \mathrm{~g} \mathrm{SDS}$ and dissolve thoroughly. Make up the final volume to $250 \mathrm{ml}$ with $\mathrm{ddH}_{2} \mathrm{O}$.

$242 \mathrm{~g}$ Tris base, $57.1 \mathrm{ml}$ glacial acetic acid and make up the final volume to 1 liter using $\mathrm{ddH}_{2} \mathrm{O}$.

$15 \mathrm{~g}$ Trizma base, $72 \mathrm{~g}$ glycine and $5 \mathrm{~g}$ SDS in 1 liter $\mathrm{ddH}_{2} \mathrm{O}$. Warm to room temperature before use if precipitation occurs. 
Blocking/ Blotting buffer

Collagenase buffer

Enzyme digestion buffer

Freezing medium

Modified HBSS buffer

Protein lysis buffer

Sodium Citrate Buffer (pH 6) Citric acid monohydrate, C6H8O7 • $\mathrm{H}_{2} \mathrm{O}$, M. wt. 210.14;

Tail lysis buffer $0.1 \mathrm{M}$ solution contains $21.01 \mathrm{~g} / \mathrm{l}$. Trisodium citrate dihydrate, C6H5O7Na3 • 2H2O, M. wt. 294.12; 0.1Msolution contains $29.41 \mathrm{~g} / \mathrm{l}$. Mix $11.5 \mathrm{ml} 0.1 \mathrm{M}$ citric acid and $88.5 \mathrm{ml} 0.1 \mathrm{M}$ trisodium citrate to make sodium citrate buffer $\mathrm{pH} 6.0$.

$5 \%$ non fat milk powder in $1 \mathrm{X}$ PBS/ $0.1 \%$ Tween 20 , at $4{ }^{\circ} \mathrm{C}$

$2 \mathrm{mg} / \mathrm{ml}$ Glucose , $2.5 \mathrm{mg} / \mathrm{ml}$ Taurine , 1\% BSA , 1.4 $\mathrm{mmol} / 1 \mathrm{MgCl}_{2}$ and $0.4 \mathrm{mg} / \mathrm{ml}$ collagenase A in HBSS.

1X PBS with $0.1 \%$ dextrose containing $5 \mathrm{mg} / \mathrm{ml}$ collagenase type II and $60 \mathrm{U} / \mathrm{ml}$ deoxyribonuclease DNAse II.

$5 \mathrm{ml} \mathrm{FBS}, 2 \mathrm{ml}$ DMSO and18 $\mathrm{ml}$ DMEM filtered through a $0.22 \mu \mathrm{m}$ strainer and stored at $4{ }^{\circ} \mathrm{C}$.

$2 \mathrm{mg} / \mathrm{ml} \mathrm{Glucose,} 2.5 \mathrm{mg} / \mathrm{ml}$ taurine, 1\% BSA, 1.4 $\mathrm{mmol} / \mathrm{l} \mathrm{MgCl}_{2}$ in HBSS buffer.

$1 \%$ Triton-X 100, $150 \mathrm{mM} \mathrm{NaCl}, 50 \mathrm{mM}$ TRIS, $5 \mathrm{mM}$ EDTA, pH 7.5, containing fresh protease $(4 \mathrm{mg} / \mathrm{ml}$ aprotinin, $4 \mu \mathrm{g} / \mathrm{ml}$ leupeptin, $4 \mu \mathrm{g} / \mathrm{ml}$ pepstatin $\mathrm{A}, 1 \mathrm{mM}$ PMSF) and phosphatase (20 mM NaF, $1 \mathrm{mM} \mathrm{Na} \mathrm{VO}_{4}, 1$ $\mathrm{mM} \mathrm{Na} \mathrm{O}_{7} \mathrm{P}_{2}$ ) inhibitors.

$200 \mathrm{mM} \mathrm{NaCl}, 50 \mathrm{mM}$ Tris/Cl pH.8, 10 mM EDTA pH. 8 and $1 \%$ SDS and $15 \mu \mathrm{l}$ of $20 \mathrm{mg} / \mathrm{ml}$ Proteinase K. 


\subsection{Cell Culture Medium and Reagents}

$\underline{\text { Product }}$

0.05\% Trypsin EDTA (1X)

96-well, round bottom suspension cells

Cell Scraper $25 \mathrm{~cm}$,

Dimethyl sulfoxide (DMSO)

DMEM

DPBS (1X) $-\mathrm{CaCl}_{2},-\mathrm{MgCl}_{2}$

Endothelial Cell Basal Medium MV Kit

Fetal Bovine Serum (FBS)

Gelatin

$\operatorname{HBSS}(1 \mathrm{X})-\mathrm{CaCl}_{2},-\mathrm{MgCl}_{2}$

Medium199 10X

NUNC multidishes 24-well plate

NUNC multidishes 6-well plate

NUNC multidishes 96-well plate

NUNC tissue culture dish $100 \times 15 \mathrm{~mm}$

NUNC tissue culture dish $60 \times 15 \mathrm{~mm}$

Nutlin-3a

Penicillin/Streptomycin (P/S)

Pifithrin- $\alpha$

Rat tail collagen type 1

Recombinant Human TGF-beta 1

Serological pipette $10 \mathrm{ml}$

Serological pipette $25 \mathrm{ml}$
Supplier

Cat. Number

Gibco®

25300-054

SARSTEDT AG \&Co.

831.837 .500

SARSTEDT AG \&Co.

Ref 83.1830

Sigma

D-5879

Invitrogen

11960

Gibco®

14190-094

PromoCell

C-22120

Gibco®

10270-106

Fluka / Sigma

48720

Gibco®

14170-112

Sigma

M0650

NUNC, Thermo Fisher

142475

Scientific Inc.

NUNC, Thermo Fisher

140685

Scientific Inc.

NUNC, Thermo Fisher

167008

Scientific Inc.

NUNC, Thermo Fisher

150679

Scientific Inc.

NUNC, Thermo Fisher

150288

Scientific Inc.

Sigma

SML0580

Sigma

P4333

Sigma

P4359

BD Bioscienses

354236

R\& D systems 240-B-002

SARSTEDT AG \&Co. $\quad$ 861.254.001

SARSTEDT AG \&Co. $\quad 861.685 .001$ 
Serological pipette $2 \mathrm{ml}$

SARSTEDT AG \&Co.

861.252 .001

Serological pipette $50 \mathrm{ml}$

SARSTEDT AG \&Co. $\quad 861.689 .001$

Serological pipette $5 \mathrm{ml}$

SARSTEDT AG \&Co. $\quad 861.253 .001$

Sterile $50 \mathrm{ml}$ filtration system, $0.22 \mu \mathrm{m}$

Millipore

SCGP00525

\subsection{Antibodies}

\subsubsection{Primary antibodies}

\begin{tabular}{|c|c|c|c|}
\hline Antigen & Antibody type & $\underline{\text { Supplier }}$ & $\underline{\text { Cat. Number }}$ \\
\hline ACTIVE® Caspase-3 & Rabbit polyclonal & Promega & G7481 \\
\hline CA IX (H-120) & Rabbit polyclonal & Santa Cruz & SC-25599 \\
\hline Carbonate dehydratase IX & Rabbit polyclonal & Bioss & bs-4029R \\
\hline CD45 (30-F11) & Rat monoclonal & Santa Cruz & SC-53665 \\
\hline $\begin{array}{l}\text { Cleaved Caspase-3 } \\
(\text { Asp175)(5A1E) }\end{array}$ & Rabbit monoclonal & Cell Signaling & 9664 \\
\hline Collagen Type I & Rabbit polyclonal & Milliore & $\mathrm{AB} 765 \mathrm{P}$ \\
\hline FSP-1 & Rabbit polyclonal & Dako & A5 5114 \\
\hline HIF $1 \alpha$ & Rabbit polyclonal & abcam & $\mathrm{ab} 2185$ \\
\hline MDM2 & Mouse monoclonal & $\begin{array}{l}\text { BD } \\
\text { Pharmingen }^{\mathrm{TM}}\end{array}$ & 556353 \\
\hline MDM2 (C-18) & Rabbit polyclonal & Santa Cruz & SC-812 \\
\hline $\mathrm{p} 21$ & Mouse monoclonal & $\begin{array}{l}\text { BD } \\
\text { Pharmingen }\end{array}$ & 556430 \\
\hline
\end{tabular}




$\begin{array}{llll}\text { p53 (1C12) } & \text { Mouse monoclonal } & \text { Cell Signaling } & 2524 \\ \text { p53 (FL-393) } & \text { Rabbit polyclonal } & \text { Santa Cruz } & \text { SC-6243 } \\ \text { PECAM-1 (MEC 13.3) } & \text { Rat monoclonal } & \text { Santa Cruz } & \text { SC-18916 } \\ \text { PE- CD31 } & \text { Mouse monoclonal } & \text { Biolegend } & 102407 \\ \text { VEGF } & \text { Rabbit polyclonal } & \text { Milliore } & \text { ABS82 }\end{array}$

\subsubsection{Secondary antibodies}

$\underline{\text { Name }}$

Alexa Fluor 488 goat anti-rabbit IgG Mo Bi Tec

$(\mathrm{H}+\mathrm{L})$

Cy3 goat anti-mouse $\operatorname{IgG}+\operatorname{IgM}(\mathrm{H}+\mathrm{L})$, Jackson affinity-purified

ECL $^{\text {TM }}$ Anti-mouse IgG, HRP linked

whole antibody( from sheep)

ECL $^{\text {TM }}$ Anti-rabbit IgG, HRP linked

whole antibody (from donkey)

FITC goat anti-mouse $\operatorname{IgG}(\mathrm{H}+\mathrm{L})$,

affinity-purified

FITC goat anti-rabbit $\operatorname{IgG}(\mathrm{H}+\mathrm{L})$,

affinity-purified

MFP488 goat anti-rat $\operatorname{IgG}(\mathrm{H}+\mathrm{L})$ $\underline{\text { Supplier }}$

Cat. Number

A11070

ImmunoResearch

GE Healthcare

NA931V

GE Healthcare

NA934V

Jackson

115-095-062

ImmunoResearch

Jackson

111-095-045

ImmunoResearch

Mo Bi Tec

MFP-A1006 
MFP555 goat anti-mouse IgG $(\mathrm{H}+\mathrm{L}) \quad$ Mo Bi Tec

MFP-A2422

MFP555 goat anti-rabbit IgG $(\mathrm{H}+\mathrm{L}) \quad$ Mo Bi Tec

MFP-A2428

\subsection{Software}

\section{Product}

AxioVision

AlphaEase FC FM $^{\text {TM }}$

BD FACS DIVA

C-View

GraphPad Prism

Image-Pro Plus

iQ5 Software

$\mathrm{KC} 4$

PIMSoft

Refenence Manager

\section{$\underline{\text { Version }}$}

Version 4.8.2

Version 4.1.0

Version 6.1.3

Version 2.1.010703

Version 5

Version 4.5.0.29

Version 170-9753

Version 3.4

Version 1.3

Version 11

\section{Company}

Carl Zeiss

Alpha Innotech

BD Biosciences

Digital Video Camera Company

GraphPad Software

Media Cybernetics

BIO-RAD

Bio-Tek

Perimed

Thomson Reuters 


\subsection{Cell Culture Experiments}

\subsubsection{Endothelial Cell Lines}

\subsubsection{Human Cardiac Microvascular Endothelial Cells}

Primary Human Cardiac Microvascular Endothelial Cells (HCMEC) were purchased from Promocell (Catalog number C-12285). HCMEC are isolated from heart ventricles from a single donor. These cells stain positive for CD31 and Willebrand factor and negative for smooth muscle alpha-actin. HCMEC closely interact with cardiomyocytes and therefore have a distinctive phenotype from other microvascular endothelial cells. HCMEC play an important role in the physiological regulation of coronary blood flow and capillary exchange.

\subsubsection{Human Umbilical Vein Endothelial Cells}

Primary Human Umbilical Vein Endothelial Cells (HUVEC) were also purchased from Promocell (Catalog number C-12200). HUVEC are isolated from the vein of the umbilical cord and are commonly used for physiological and pharmacological investigations, such as macromolecule transport, blood coagulation, angiogenesis, and fibrinolysis.

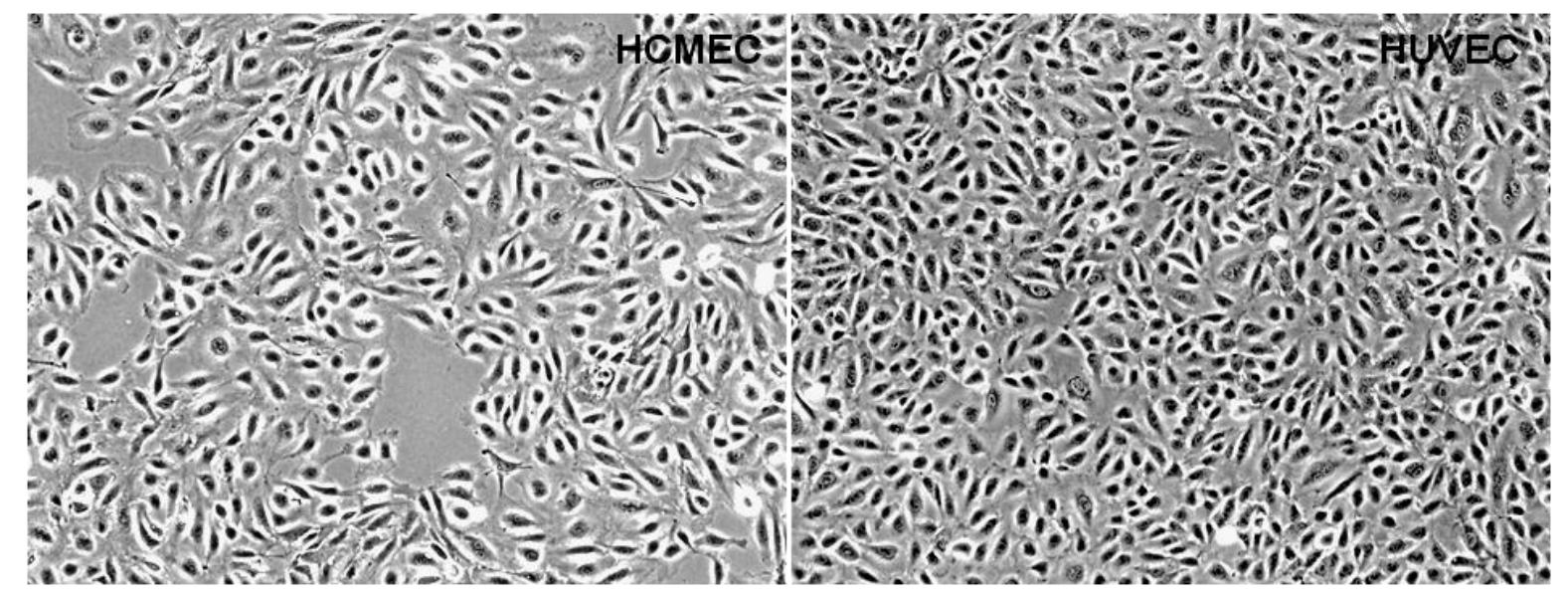

(Figure adopted from ScienCell Research Laboratory.)

Figure 2.1. Microscopic image of HCMEC and HUVEC. HCMECs isolated from human heart and HUVECs isolated from human umbilical veins, Phase contrast, 100X. 


\subsubsection{Cardiac Endothelial Cell Isolation}

Coronary microvascular endothelial cells (CMECs) were isolated according to the methods published previously pr. ${ }^{115}$ Hearts were removed from 4 mice after cervical dislocation under $2 \%$ isoflurane anaesthesia and placed into ice-cold PBS. Fresh harvested hearts were immediately dipped into $70 \%$ ethanol to devitalize membranes and back to cold PBS. Fat, connective, vascular and valve/atrial tissue were removed from the hearts followed by cutting the hearts open to wash away the blood. The hearts were chopped into small pieces (1-2 $\mathrm{mm}^{3}$ ) in $25 \mathrm{ml}$ modified HBSS buffer and spun at 500rpm for $1 \mathrm{~min}$. The pellet of the fragments was digested in $5 \mathrm{ml}$ collagenase buffer for $5 \mathrm{~min}$ at $37^{\circ} \mathrm{C}$ with shaking every 1 min. The collagenase digestion was repeated 4 times with supernatant taken out after each digestion. Trypsin digestion was subsequently applied on the remaining pellet with $5 \mathrm{ml}$ trypsin buffer for $5 \mathrm{~min}$ at $37^{\circ} \mathrm{C}$ with shaking every $1 \mathrm{~min}$ and repeated 4 times. In this case, heart segments were digested with collagenase to remove myocytes and fibroblasts and subsequently with trypsin to free endothelial cells and smooth muscle cells. After each trypsin digestion, the supernant $(\sim 5 \mathrm{ml})$ was taken into a tube containing $1 \mathrm{ml} \mathrm{FCS}$ and combined at the end of the digestion, to which another $15 \mathrm{ml} \mathrm{CMEC} \mathrm{medium} \mathrm{was} \mathrm{added} \mathrm{and} \mathrm{centrifuged}$ at $1200 \mathrm{rpm}$ for $5 \mathrm{~min}$. The pellet was resuspended in CMEC medium and seeded into $1 \%$ gelatin coated T75 flask. After incubating for 2 hours and 24 hours at $37^{\circ} \mathrm{C}$ with $5 \% \mathrm{CO}_{2}$, the medium and non-adherent cells (fibroblasts and blood cells) were removed and replaced with fresh CMEC medium. CMECs were cultured in CMEC medium (FCS 10\%, Penicillin/Streptomycin $100 \mu \mathrm{g} / \mathrm{ml}$, L-Glutamine $2 \mathrm{mmol} / \mathrm{l}$, Endothelial cell growth supplement $10 \mu \mathrm{g} / \mathrm{ml}$, heparin $5 \mu \mathrm{g} / \mathrm{ml}$, hydrocortisone $1 \mu \mathrm{g} / \mathrm{ml}$, 2-mercaptoethanol $1 \mu \mathrm{mol} / \mathrm{L}$, mEGF $10 \mathrm{ng} / \mathrm{ml}$, ascorbic acid $1 \mu \mathrm{g} / \mathrm{ml}$, VEGF $0.5 \mathrm{ng} / \mathrm{ml}$, IGF-1 $10 \mathrm{ng} / \mathrm{ml}$ in $1000 \mathrm{mg} / \mathrm{L}$ glucose DMEM) and medium was changed every two days. CMECs were used for experiment at passage 1-2.

To isolate cardiac CD31-positive and -negative cells, cells were subsequently surface labeled using CD31-PE antibodies and sorted using a BD FACSaria II cell sorter. 


\subsubsection{Generation of Stable p53 Knockdown Cell Line}

Deletion of p53 from endothelial cells were done in collaboration with Xingbo $\mathrm{Xu}$ and Xiaopeng Liu, members of Prof. Dr. med. Elisabeth Zeisberg lab. We have used lentivirus based Human p53 shRNA expression vectors or scrambled non-effective shRNA cassette (in pGFP-C-shLenti plasmid) obtained from Amsbio to generate a stable p53 knockdown endothelial cell line. Lentivirus packaging was performed as previously described $\left(\right.$ Streckfuss-Bömeke et al., 2013) ${ }^{116}$. Briefly, Human Embryonic Kidney 293T (HEK293T) cells were plated at $4 \times 10^{6}$ cells per $100 \mathrm{~mm}$ dish and incubated overnight. Cells were transfected with $10 \mu \mathrm{g}$ of pLenti-shp53 along with $2.5 \mu \mathrm{g}$ of Virus packaging plasmid mix by using Lipofectamine 2000 (Invitrogen), according to the manufacturer's instructions. Virus collection was performed two times at 48 hours and 72 hours after transfection, the supernatant of transfectant was collected and filtered through a $0.22 \mu \mathrm{m}$ pore-size cellulose acetate filter (Millipore). Human Cardiac Microvascular Endothelial Cells (PromoCell) were seeded at $2 \times 10^{5}$ cells per $100 \mathrm{~mm}$ dish 1 day before transduction. The medium was replaced with virus-containing supernatant supplemented with $5 \mu \mathrm{g} / \mathrm{ml}$ polybrene (Sigma), and incubated for 12 hours.

\subsubsection{Modulation of $\mathrm{p} 53$ and Induction of EndMT in vitro}

$\mathrm{HCMECs}$ were cultured at $37^{\circ} \mathrm{C}$ in humidified air with $5 \% \mathrm{CO}_{2}$ on gelatine-coated $100 \mathrm{~mm}$ culture dishes in endothelial cell basal medium supplemented with $2 \% \mathrm{FCS}, 5 \mathrm{ng} / \mathrm{ml}$ epidermal growth factor, $22.5 \mu \mathrm{g} / \mathrm{ml}$ heparin and $1 \mu \mathrm{g} / \mathrm{ml}$ hydrocortisone. Medium was changed every 2 days. After the culture reached $80 \%$ of confluent density, the cells were subcultured with a ratio of 1:4. For EndMT induction, HCMECs were serum-starved and incubated with $10 \mathrm{ng} / \mathrm{ml}$ TGF $\beta 1$ for 6 or 12 days, with nutlin-3a and pifithrin- $\alpha$ being supplied every other day. 


\subsubsection{Endothelial Cell Functionality Assays}

\subsubsection{In vitro 3-D Spheroid Angiogenesis Assay}

The 3-dimensional spheroid angiogenesis assay was established by Thomas Korff ${ }^{117}$. It is a functional assay to test whether cells can form a vascular structure. This assay consists of two main steps:

\subsection{Generation of Spheroids}

To generate spheroids, HCMVCs were detached and resuspended in Endothelial cell growth medium containing $20 \%$ methyl cellulose solution. The stock solution of methyl cellulose was prepared by dissolving $7 \mathrm{~g}$ of methyl cellulose (pre autoclaved) in preheated $500 \mathrm{ml}$ of M199 medium $\left(60^{\circ} \mathrm{C}\right)$. The solution was mixed for 30 minutes using a magnetic stirrer, and stored at $4^{\circ} \mathrm{C}$ overnight. Penicillin/Streptomycin $(\mathrm{P} / \mathrm{S})$ was added to the methyl cellulose stock solution and stored in $50 \mathrm{ml}$ aliquots. The solution was spun down at $4{ }^{\circ} \mathrm{C}$ for 2 hours at $4000 \mathrm{rpm}$ to remove the precipitate or undissolved methyl cellulose. The clear gel-like supernatant after centrifugation, was used for experiments. Cells were counted and adjusted to a concentration of $4 \times 10^{4}$ cells in $10 \mathrm{ml}$ (for one 96-well plate) of growth medium containing $20 \%$ methyl cellulose solution. Cells suspension $(100 \mu \mathrm{l} /$ well $)$ was seeded in nonadherent round-bottom 96-well plates using a 12-channel pipette and incubated at $37^{\circ} \mathrm{C}$. After 24 hrs of incubation, cells formed a single spheroid.

\subsection{Culture in Collagen Type I}

After 24 hours of incubation, spheroids were harvested using $1000 \mu$ l pipette tip and embedded into collagen gels. A pre- warmed humidified 24-well culture plates were prepared in advance in $37^{\circ} \mathrm{C}$ incubator.

Before harvesting the spheroids, methylcellulose stock solution with 20\% FBS and type I rat tail collagen diluted 1:1 with filtered $0.1 \%$ acetic acid and mixed with 10X M199 medium and neutralized filtered $0.2 \mathrm{~N} \mathrm{NaOH}$ immediately before use. Spheroids were harvested carefully without disturbing the spheroid structures. After centrifugation at $1200 \mathrm{rpm}$ for 3 minutes, spheroid pellets were overlaid with methylcellulose stock solution and gently diluted 
equal amount with neutralized collagen working solution. The mixed spheroid containing gel was quickly transferred in duplicates into pre-warmed 24-well plates by adding $1 \mathrm{ml}$ (containing approximately 40 spheroids) to each well and incubated at $37^{\circ} \mathrm{C}$ for 30 minutes. $100 \mu \mathrm{l}$ of culture medium were added to each well after solidification of the collagen, and incubated in a $37^{\circ} \mathrm{C}$ incubator for at least 24 hours. The total sprout length and no. of sprouts of 15-20 randomly selected spheroids was measured to calculate mean sprout length and mean no. of sprouts for each group. Pictures were taken using AxioVision software and data were evaluated by Image-Pro Plus. Total sprout length means the length of all sprouts grown out of an individual spheroid.

\subsubsection{Freezing and Thawing of Cells}

Cells to be preserved were detached by T/E solution at RT until all the cells had detached from the surface of the dishes. Trypsin was neutralized by the addition of cell type-dependent culture medium. Cells were spun down at $1200 \mathrm{rpm}$ for $5 \mathrm{~min}$, the supernatant was discarded and the cell pellets were resuspended in freezing medium. The cell suspension was gently mixed and transferred into polypropylene cryovials ( $1 \mathrm{ml}$ per vial). The vials were transferred to a freezing container and kept at $-80^{\circ} \mathrm{C}$ at least overnight before being transferred to liquid nitrogen storage tanks for long-term storage.

For thawing the frozen cells, cells in cryovials were thawed at $37^{\circ} \mathrm{C}$ in a water bath for approximately 90 seconds and transferred to a $15 \mathrm{ml}$ centrifuge tube containing $10 \mathrm{ml}$ of prewarmed $\left(37^{\circ} \mathrm{C}\right)$ growth medium. Cells were centrifuged at $1500 \mathrm{rpm}$ for 5 minutes and the supernatant was discarded to remove the freezing medium. Cell pellets were again resuspended gently with pre-warmed growth medium and then transferred to new culture dishes coated with $0.1 \%$ gelatin. Cell cultures were incubated at $37^{\circ} \mathrm{C}$ in humidified air with $5 \% \mathrm{CO}_{2}$. 


\subsection{Experiments in Mice}

\subsubsection{Experimental Animals}

To generate mice with inducible endothelial cell-specific p53 deletion (End.p53-KO), mice with loxP-flanked (floxed, $f l$ ) p53 alleles (courtesy of Arnold Berns; C57BL/6 background) ${ }^{118}$ were mated with mice expressing a Cre recombinase-estrogen receptor fusion protein ER(T2) under control of the endothelial receptor tyrosine kinase (Tie2) promoter (courtesy of Berndt Arnold; C57BL/6 background). ${ }^{119}$ This inducible Tie2.Cre mouse line has been previously shown to allow efficient temporal gene deletion exclusively in endothelial cells, including the heart, whereas the percentage of recombined cells was found to be negligible in hematopoeitic cells. ${ }^{119}$ Cre recombinase activity was induced by feeding 5 to 6 week-old mice with rodent chow containing $400 \mathrm{mg} / \mathrm{kg}$ tamoxifen citrate (TD55125; Harlan Teklad) for 6 weeks. ${ }^{119,118 ; 120}$ Successful p53 gene excision was confirmed by PCR analysis of genomic DNA from tail biopsies. ${ }^{118,119}$ Cre-wildtype (WT) $x$ p5 $3_{\mathrm{fl} / \mathrm{fl}}$ mice fed tamoxifen chow and Cre-tg x p53 fl/fl mice on normal rodent chow were used as controls (End.p53-WT). Ageand gender-matched littermates were used throughout the study. All animal care and experimental procedures had been approved by the institutional Animal Research Committee and complied with national guidelines for the care and use of laboratory animals.

\subsubsection{Transverse Aortic Constriction}

Female mice were anesthetized via $2 \%$ isoflurane inhalation and subjected to minimally invasive TAC surgery over a 26-gauge needle. ${ }^{121}$ Anesthesia depth was monitored by observing the respiratory rate and the toe-pinch reflex test. Sham-operated mice, in which the aortic arch was exposed, but not ligated, were also examined. Three days after surgery, the pressure gradient over the aortic ligature was determined using pulsed wave Doppler. Echocardiography was performed before and at tissue harvest 8 and 20 weeks after TAC. At tissue harvest, hearts were rapidly excised and weighed. Next, the atria were removed and the ventricles immediately divided into two parts. The top half of each heart was placed in saline at $4^{\circ} \mathrm{C}$ for cryoprotection, subsequently embedded in tissue freezing medium and processed for histological analysis. The bottom half of each heart was stored at $-80^{\circ} \mathrm{C}$ and subsequently used for RNA and protein isolation, or endothelial cell separation, respectively. 


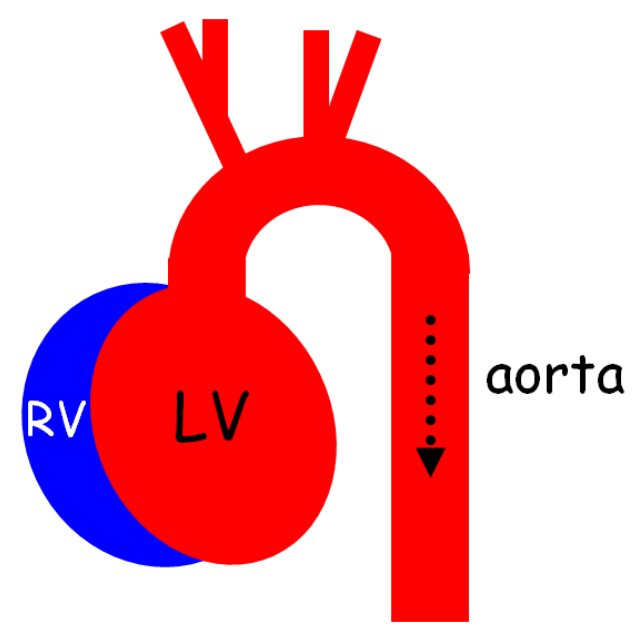

Before TAC

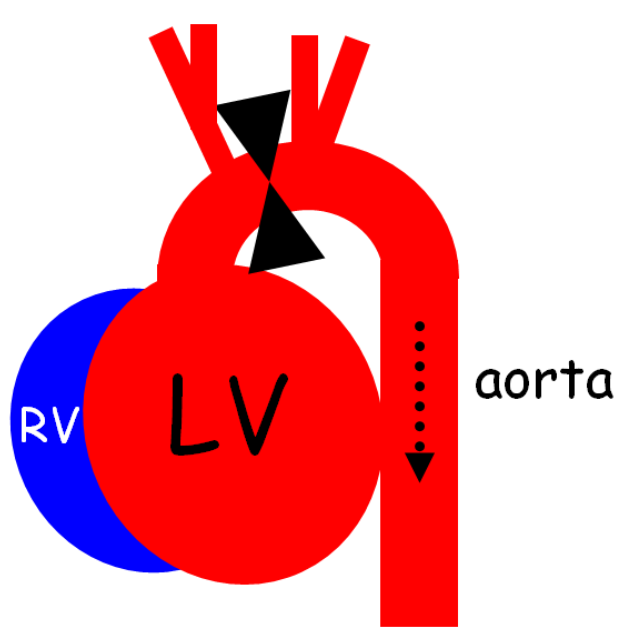

After TAC

(figure, kind courtesy of PD Dr. med. Karl Toischer)

Figure 2.2. Transverse Aortic Constriction. Representative cartoon showing the increase in left ventricle size in response to increased afterload.

\subsubsection{Doxorubicin Injection}

End.p53-WT and End.p53-KO male mice aged 11-15 weeks were injected intraperitoneally (i.p.) with a single dose of either doxorubicin hydrochloride (Sigma; $20 \mathrm{mg} / \mathrm{kg} \mathrm{BW}$ in $150 \mu \mathrm{L}$ saline) or saline. Echocardiography was performed before and at tissue harvest 5 days or 8 weeks. Mice were sacrificed by cervical dislocation after 5 days or 8 weeks post Dox injection and heart tissues were subsequently collected, weighed and divided into two parts. The top half of each heart was placed in saline at $4^{\circ} \mathrm{C}$ for cryoprotection, subsequently embedded in tissue freezing medium and processed for histological analysis. The bottom half of each heart was stored at $-80^{\circ} \mathrm{C}$. Each treatment group consisted of five to seven mice.

\subsubsection{Transthoracic Echocardiography}

Two-dimensional (2D) directed echocardiography was performed by a blinded examiner before and the indicated time points after surgery in mice under $1.5 \%$ isoflurane anesthesia using the Vevo 2100 system (Visualsonics) equipped with a $30 \mathrm{MHz}$ center frequency ultrasound transducer. ${ }^{121}$ Typically, heart rates were $460 \pm 15$ beats/minute, respiratory rates were $125 \pm 3.8$ breaths/minute. Original M-mode recordings were used to determine the end- 
diastolic (EDD) and end-systolic (ESD) LV chamber diameter and posterior wall thickness (PWTh). Echocardiographic LV mass (LVM) was estimated using the formula: $1.055 \mathrm{x}$ $\left([\mathrm{AWTh}+\mathrm{EDD}+\mathrm{PWTh}]^{3}-\mathrm{EDD}^{3}\right) . \mathrm{LV}$ fractional shortening (FS) was calculated as (EDD ESD) / EDD x 100.

\subsubsection{Unilaterial Hindlimb Ischemia}

Ten to twelve week-old male End.p53-WT and End.p53-KO mice were used to examine the neovascularization in vivo using the unilateral hindlimb ischemia mouse model. ${ }^{122 ; 123}$ End.p53-WT mice without tamoxifen food were also examined simultaneously to analyze the effect of tamoxifen in reperfusion after hindlimb ischemia. After anesthesia by intraperitoneal injection of xylazine / ketamine hydrochloride, unilateral hindlimb ischemia was induced by permanent ligation of the right femoral artery (immediately distal to the origin of the deep femoral artery) as well as the distal portion of the saphenous artery with 6-0 silk sutures. Perfusion was determined before and immediately after ischemia induction as well as on day 7, 14, 21 and 28 after surgery using laser Doppler perfusion imaging. Perfusion in the contralateral hindlimb was used as internal control (set at 100\%).
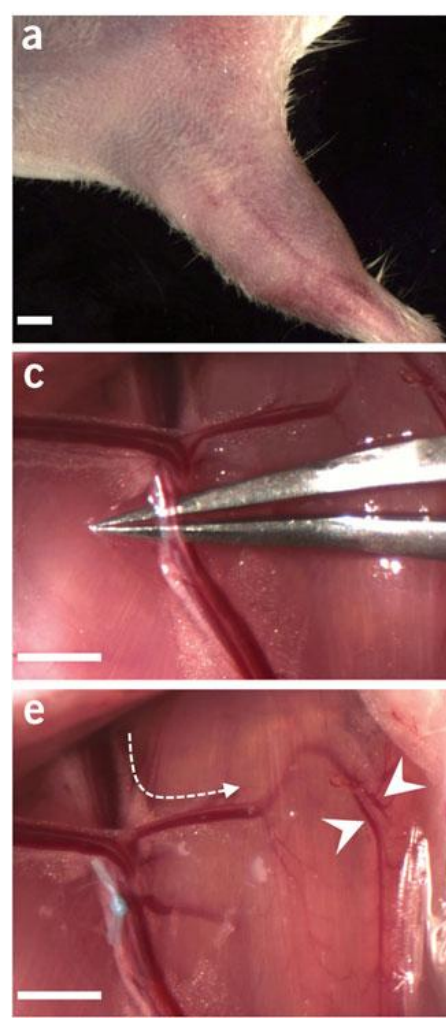
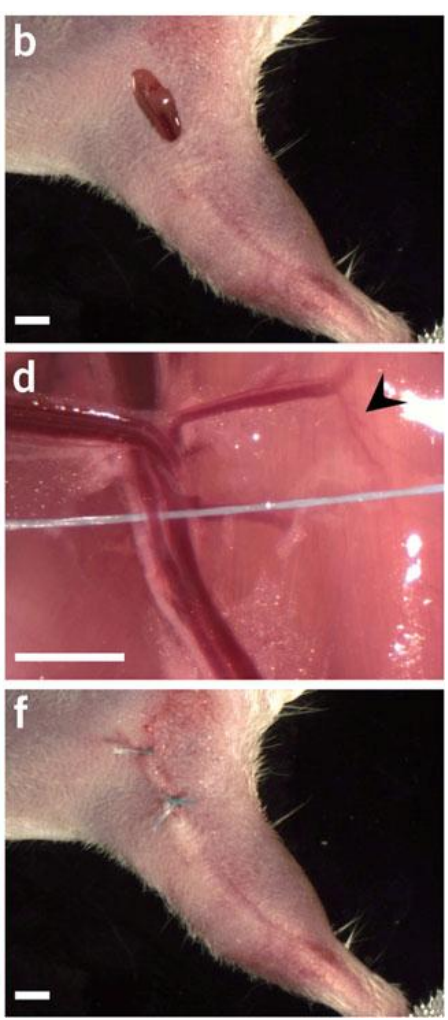

Figure 2.3. Unilaterial Hindlimb Ischemia: Ventral aspect of right mouse thigh and upper hind limb after hair removal (a). Surgical skin incision (5 $\mathrm{mm}$ ) beginning from the groin skinfold following and immediately lateral to the vascular contours visible through the skin (b). Blunt dissection and separation of the femoral artery and vein (c). Insertion of a surgical thread underneath the femoral artery (d). Faint appearance of collaterals from the deep branch (arrowhead). Ligation of the femoral artery distal to the origin of the deep branch by a triple surgical knot (e). Blood flow is diverted to collaterals (dashed white arrow) and collateral arteries now appear prominent (compare arrowheads from d and e). Over-andover skin suture (f). Scale bars, $2 \mathrm{~mm}$.

(Figure adapted from Limbourg A et al. Nat Protoc 2009;4:1737-46) 


\section{Histochemistry}

Histochemical analyses were performed on $5 \mu \mathrm{m}$-thick frozen, acetone-fixed cross sections through the LV of mice 8 and 20 weeks after TAC, or through the lower hindlimb of mice 4 weeks after femoral artery ligation. Cardiac fibrosis was determined by Masson's trichrome (MTC) staining. Interstitial collagen was visualized using picrosirius red staining. Capillary endothelial cells were assessed after incubation with monoclonal antibodies against CD31 followed by Cy3-labeled secondary antibodies. Cell nuclei were visualized using 4',6diamidino-2-phenylindole (DAPI). In some mice, the functionality of cardiac vessels was assessed by intracardiac (i.c.) injection of endothelial fluorescein (FITC)-conjugated Griffonia simplicifolia isolectin B4 (GSL-I; $50 \mu \mathrm{g}$ in $200 \mu \mathrm{l}$ saline) $15 \mathrm{~min}$ prior to sacrifice with an overdose of isoflurane. Cells not positive following CD31 antibody incubation or endothelial cell-lectin perfusion are denoted as 'CD31-negative cells' or 'lectin-negative cells', respectively. Cardiomyocyte membranes were visualized using FITC-labeled wheat germ agglutinin (WGA) followed by determination of the minimal single cardiomyocyte cross-sectional area (CSA). Per cross section, at least 10 randomly selected cardiomyocytes were evaluated and results averaged. Cardiac apoptosis was examined using Terminal deoxynucleotidyl transferase dUTP nick end labeling (TUNEL) or antibodies against activated caspase-3 and p53. A rabbit anti-carbonic anhydrase IX (CAIX) antibody was used as surrogate marker for hypoxia. ${ }^{124}$ Fibroblast-specific protein (FSP)-1 was detected using a polyclonal rabbit anti-mouse antibody. All morphometric analyses were performed using image analysis software.

\section{Molecular Biology Methods}

\subsection{Genomic DNA Isolation from Mouse Tail}

Mouse tail biopsies were used to isolate genomic DNA. Tail biopsies were lysed using tail lysis buffer. Tail biopsies with tail lysis buffer $(600 \mu \mathrm{l})$ was incubated at $56^{\circ} \mathrm{C}$ overnight with shaking (600 rpm). $200 \mu \mathrm{l}$ of saturated $\mathrm{NaCl}(2 \mathrm{M})$ was added to lysate and centrifuged at 15 $\mathrm{krpm}$ (max. speed) for $30 \mathrm{~min}$ at $4^{\circ} \mathrm{C}$. Then, $750 \mu \mathrm{l}$ of supernatant were transferred to a fresh $1.5 \mathrm{ml}$ tube and $450 \mu \mathrm{l}$ of cold isopropanol added, mixed gently and centrifuged at $15 \mathrm{krpm}$ for $30 \mathrm{~min}$ at $4^{\circ} \mathrm{C}$. Supernatant was discarded and pellet was washed with $500 \mu \mathrm{l}$ of $70 \%$ 
EtOH and centrifuged $10 \mathrm{krpm}$ for $15 \mathrm{~min}$ at $4^{\circ} \mathrm{C}$. Finally, the gDNA pellet was air dried at $\mathrm{RT}$ and resuspended in 20-30 $\mu 1$ of nuclease free water.

Genomic DNA from the mouse tails were used for genotyping. The mouse carrying the p53 flox alles were identified by PCR using the following primers: primer A: p53-int1-fwd:5'CACAAAAACAGGTTAAACCCAG, primer B: p53-int1-rev: 5'-AGCACATAGGAGGCA GAGAC, primer C: p53-int 10-fwd: 5'-AAGGGGTATGAGGGACAAGG; primer D: 5'-GA AGACAGAAAAGGGGAGGG and for Cre the primers are Cre1 5' CGAGTGATGAGGTT CGCAAG and Cre2 5' TGAGTGAACGAACCTGGTCG. The PCR condition for p53 flox alleles and cre is $95^{\circ} \mathrm{C}$ for $2 \mathrm{~min}, 95^{\circ} \mathrm{C}$ for $30 \mathrm{sec}, 58^{\circ} \mathrm{C}$ for $30 \mathrm{sec}, 72^{\circ} \mathrm{C}$ for $1 \mathrm{~min}$, reapeat for 45 cycles, $72^{\circ} \mathrm{C}$ for $10 \mathrm{~min}$ and soak at $4^{\circ} \mathrm{C}$. The PCR product size of p53 intron 1, intron 10 and cre are $288 \mathrm{bp}, 431 \mathrm{bp}$ and no band for wild type mice and $370 \mathrm{bp}, 584 \mathrm{bp}$ and $390 \mathrm{bp}$ for p53 flox/ flox + cre heterozygous mice respectively.

\subsection{Isolation of Total RNA from Heart Tissue}

RNA was extracted using the TRI Reagent ${ }^{\circledR}$ Solution (Ambion) according to the manufacturer's instructions. The tissue samples were homogenized in 10-20 volumes of TRI Reagent ${ }^{\circledR}$ solution. The homogenate was incubated for $10 \mathrm{~min}$ at RT. Then, $100 \mu \mathrm{l}$ of chloroform per $1 \mathrm{ml}$ of TRI Reagent solution was added, mixed well and incubated at room temp for $10 \mathrm{~min}$. Then, the homogenate was spun down at $12,000 \mathrm{x} \mathrm{g}$ for $15 \mathrm{~min}$ at $4{ }^{\circ} \mathrm{C}$, and the aqueous phase was transferred to a fresh tube. $500 \mu \mathrm{l}$ of isopropanol per $1 \mathrm{ml}$ of TRI Reagent solution was added to aqueous sample to precipitate the nucleic acids. The sample was incubated at RT for $10 \mathrm{~min}$. Then, the sample was centrifuged at $12,000 \mathrm{x} \mathrm{g}$ for $8 \mathrm{~min}$ at $4^{\circ} \mathrm{C}$. The supernatant was removed without disturbing the pellet. $1 \mathrm{ml}$ of $75 \%$ ethanol per 1 $\mathrm{ml}$ of TRI Reagent solution was added to each sample to wash the RNA pellets and then centrifuged at 7,500 $\mathrm{x}$ g for $5 \mathrm{~min}$ at $4^{\circ} \mathrm{C}$. The ethanol was removed without disturbing the pellet. Residual ethanol was removed by centrifuging again briefly and removing the remaining ethanol with a fine tip pipette. RNA pellet was air dried for 3-5 min and resuspended in 20-30 $\mu 1$ of nuclease free water or nuclease-free TE buffer. 


\subsection{Determination of RNA Quality and Concentration}

The quality and concentration of RNA was determined by NanoDrop® ND 1000. The ratio of absorbance at $260 \mathrm{~nm}$ and $280 \mathrm{~nm}$ is used to assess the purity of RNA. A ratio of $\sim 2.0$ is generally accepted pure for RNA. If the ratio is lower, it may indicate the presence of protein, phenol or other contaminants that absorb strongly at or near $280 \mathrm{~nm}$. The resultant 260:280 ratio for the nucleic acid being studied will be approximately equal to the weighted average of the 260/280 ratios for the four nucleotides present. For nucleic acid quantification, a modification of the Beer-Lambert equation is used to calculate sample concentration $(\mathrm{C}=(\mathrm{A}$ $\left.{ }^{*} \varepsilon\right) / \mathrm{b}$ ), where $\mathrm{C}$ is nucleic acid concentration in $\mathrm{ng} / \mu \mathrm{l}, \mathrm{A}$ is absorbance in $\mathrm{AU}, \varepsilon$ is the wavelength dependent extinction coefficient in $n g-\mathrm{cm} / \mu \mathrm{l}$ and $\mathrm{b}$ is the pathlength in $\mathrm{cm}$.

\subsection{Reverse Transcription Polymerase Chain Reaction (RT-PCR)}

RT or cDNA synthesis was performed using the M-MLV Reverse Transcriptase. A total of 1 $\mu \mathrm{g}$ total RNA was used for each RT reaction together with random hexamer primers, RiboSafe RNase Inhibitor, $\mathrm{MgCl}_{2}$ and dNTPs. The final volume of RT reaction was $20 \mu \mathrm{l}$. In an RNase-free $0.2 \mathrm{ml}$ microcentrifuge tube, the following reagents were prepared on ice.

\section{cDNA synthesis}

$\begin{array}{ll}\text { Total RNA } 1 \mu \mathrm{g} & \mathrm{x} \mu \mathrm{l} \\ \text { M-MLV RT 5X buffer } & 4 \mu \mathrm{l} \\ \text { dNTPs } & 2 \mu \mathrm{l} \\ \mathrm{MgCl}_{2} & 4 \mu \mathrm{l} \\ \text { RNase Inhibitor 40 U/ } \mu \mathrm{l} & 0.6 \mu \mathrm{l} \\ \text { Random primers } & 0.2 \mu \mathrm{l} \\ \text { Reverse Transcriptase, 200 U } & 0.4 \mu \mathrm{l} \\ \text { DEPC water } & 8.8-\mathrm{x} \mu \mathrm{l} \\ \text { Final volume } & \mathbf{2 0} \boldsymbol{\mu l}\end{array}$


The mixtures of $20 \mu 1$ final volumes was mixed by gentle pipetting and then spun down. The contents were incubated at $42^{\circ} \mathrm{C}$ for $20 \mathrm{~min}$. RT reaction was inactivated by heating to $99^{\circ} \mathrm{C}$ for $5 \mathrm{~min}$. The cDNA was stored at $-20^{\circ} \mathrm{C}$ or directly used as a template for PCR amplification.

PCR was performed to standardize the primers to optimize amplification condition with GoTaq Polymerase using $2 \mu 1$ of cDNA per reaction. Amplification reactions were performed in an automatic thermocycler equipped with a heatable lid. The following reagents were prepared and added to $0.2 \mathrm{ml}$ microcentrifuge tubes on ice:

$\begin{array}{lc}\text { cDNA } & 2 \mu \mathrm{l} \\ \text { DEPC water } & 13 \mu \mathrm{l} \\ \text { 5X GoTaq buffer } & 5 \mu \mathrm{l} \\ \mathrm{MgCl}_{2}, 25 \mathrm{mM} & 1.8 \mu \mathrm{l} \\ \text { Sense primer, } 5 \mu \mathrm{M} & 1.5 \mu \mathrm{l} \\ \text { Antisense primer, } 5 \mu \mathrm{M} & 1.5 \mu \mathrm{l} \\ \text { GoTaq DNA polymerase, } 50 \mathrm{U} / \mu \mathrm{l} & 0.2 \mu \mathrm{l} \\ \text { Final volume } & \mathbf{2 5} \mu \mathrm{l}\end{array}$

Mixtures were gently mixed and spun down prior to reaction. Primer sequences, annealing and extension conditions are shown in section 4.7 and had been carefully established previously. Of note, the number of PCR cycles for each set of primers was verified to be in the linear range of the amplification. The initialization step is heating the reaction to a temperature of $95^{\circ} \mathrm{C}$ for $1-3$ min to ensure the template DNA is completely denatured. The final elongation step is performed at a temperature of $72^{\circ} \mathrm{C}$ for $10 \mathrm{~min}$ after the last PCR cycle to ensure the remaining single-stranded DNA is fully extended. A standard PCR program is shown as follows: 


$\begin{array}{ll}\text { Initial denaturation } & 95^{\circ} \mathrm{C} \text { for } 3 \mathrm{~min}, 1 \text { cycle } \\ & \text { followed by } 30-40 \text { cycles of; } \\ \text { Denaturation } & 94^{\circ} \mathrm{C} \text { for } 30 \mathrm{sec} \\ \text { Annealing } & \text { For the temperature, refer to (section 4.7), } \\ & 30 \mathrm{sec} \\ \text { Extension } & 72^{\circ} \mathrm{C}, \text { time depends on the size of PCR } \\ & \text { product, } 1 \mathrm{~kb} / \mathrm{min} \\ \text { Final extension } & 72^{\circ} \mathrm{C} \text { for } 10 \mathrm{~min}, 1 \text { cycle } \\ \text { Soak } & 4^{\circ} \mathrm{C}\end{array}$

Products were stored at $4{ }^{\circ} \mathrm{C}$ until analysis. $20 \mu \mathrm{l}$ of PCR products were analyzed on 1.5 or $2 \%$ agarose gels and visualized by ethidium bromide.

\subsection{Quantitative real-time PCR}

Real-time PCR (qRT-PCR) was used to amplify cDNA products to study and compare gene expression between groups. SYBR ${ }^{\circledR}$ Green PCR Master Mix was used to detect and quantify PCR products in real-time reactions ${ }^{125}$. SYBR Green is a fluorescent intercalating dye which binds to double-stranded DNA and upon excitation emits light. ${ }^{126}$ Thus, as a PCR product accumulates, fluorescence increases. Real-time PCR record fluorescence and report the results as a $\mathrm{Ct}$ (cycle threshold). The $\mathrm{Ct}$ is defined as the number of cycles required for the fluorescent signal to cross the threshold. This threshold is a fluorescence value slightly above the background fluorescence measured before exponential growth starts.

Total RNA was isolated using TRI Reagent ${ }^{\circledR}$ solution, and the amount and quality checked by Nanodrop. One $\mu \mathrm{g}$ RNA was reversed transcribed into cDNA, followed by quantitative PCR using real-time assessment of $\mathrm{SYBR}^{\circledR}$ Green and the iCycler iQ Detection system. qPCR primers and conditions are listed in section 4.7. Starting quantities were extrapolated from standard curves for each primer set. 
The following reagents were prepared and added to a 96 well-plate on ice:

$\begin{array}{lc}\text { qPCR reaction } & \\ \text { 2X SYBR Green PCR Master Mix } & 10 \mu \mathrm{l} \\ \text { cDNA } & 2 \mu \mathrm{l} \\ \text { DEPC water } & 5 \mu \mathrm{l} \\ \text { Sense primer, } 5 \mu \mathrm{M} & 1.5 \mu \mathrm{l} \\ \text { Antisense primer, } 5 \mu \mathrm{M} & 1.5 \mu \mathrm{l} \\ \text { Final volume } & \mathbf{2 0} \boldsymbol{\mu l}\end{array}$

Duplicate aliquots (each $20 \mu \mathrm{l}$ ) were carefully added into the appropriate wells and the plate was sealed with adhesive film. After a brief centrifuge spin to distribute the mixtures to the bottoms of the wells, qPCR reaction was performed using default conditions, with exception of the melting temperature and the number of cycles which were altered according to the primer set used in the particular reaction:

Initial denaturation

Annealing and extension

Disassociation/melting curve

Soak $50^{\circ} \mathrm{C}$ for 2 min followed by $95^{\circ} \mathrm{C}$ for

$10 \mathrm{~min}, 1$ cycle

$95^{\circ} \mathrm{C}$ for $15 \mathrm{sec} ; 60^{\circ} \mathrm{C}$ for $1 \mathrm{~min}, 35-40$ cycles

$15 \mathrm{sec}$ each at 95,60 , and $95^{\circ} \mathrm{C}$

$4^{\circ} \mathrm{C}$

The specificity of the SYBR Green assay was verified by melting curve analyses and the results were analyzed by comparative $\mathrm{C}_{\mathrm{t}}$ method ${ }^{127}$, with:

$$
\begin{aligned}
{[\text { delta }][\text { delta }] C_{t} } & =[\text { delta }] C_{t, \text { sample }}-[\text { delta }] C_{t, \text { reference }} \\
\text { Fold change } & =2^{\text {[delta] }] \text { delta }] \mathrm{Ct}}
\end{aligned}
$$

All qPCR data (two or more biological replicates with three technical replicates each) were calculated using the delta delta $\mathrm{Ct}$ method and normalized to glyceraldehyde-3-phosphate dehydrogenase (Gapdh) RNA, and are reported as -fold change vs. sham-operated mice. 


\subsection{Agarose Gel Electrophoresis Detection of PCR Amplicons}

1.5 or $2 \%$ agarose gels were prepared depending upon the size of DNA fragment. For this purpose, the required amount of agarose was measured and $1 \mathrm{X}$ TAE buffer was added. Agarose was dissolved by boiling in a microwave oven until the solution appeared clear. Agarose solution was allowed to cool down till $60^{\circ} \mathrm{C}$ and ethidium bromide was added to a concentration of $0.5 \mu \mathrm{g} / \mathrm{ml}$. The gels were cast in the casting chamber and were allowed to polymerize at RT. $20 \mu \mathrm{l}$ of PCR products and DNA ladder were loaded in the wells. Electrophoresis was performed at 80-100 volts and DNA was visualized in the gel by addition of ethidium bromide and placed on an UV transilluminator. Ethidium bromide binds to both single- and double-stranded nucleic acids (DNA and RNA) by intercalating between the bases ${ }^{128}$ and is fluorescent, meaning that it absorbs invisible UV light and transmits the energy as visible orange light. 


\subsection{Primer Sequences and qRT-PCR Conditions}

Gene Primer sequence (in $5^{6}-3^{6}$ direction)

Bmp7 F: ACCCTTCATGGTGGCCTTCT

R: CCTCAGGGCCTCTTGGTTCT

CD31

F: GCGCAGGACCACGTGTTAGT

R: CCTGCAATTTGAATCCGGAC

Colla1 F: ATGGATTCCCGTTCGAGTACG

R: TCAGCTGGATAGCGACATCG

Ctgf

F: CTTCTGCGATTTCGGCTCC

R: TACACCGACCCACCGAAGA

Gapdh

F: GCACAGTCAAGGCCGAGAAT

R: GCCTTCTCCATGGTGGTGAA

Mdm2 F: GCGCTTCCTAGGTCACTTTG

60

40

R: CCAGCAACTGTTCAGACGAA

Mmp2

F: ACCCAGATGTGGCCAACTAC

R: TACTTTTAAGGCCCGAGCAA

Mmp9 F: GAGCTGTGCGTCTTCCCCTTC

58

40

R: GGAATGATCTAAGCCCAGTGC

p21

F: CCTGACAGATTTCTATCACTCCA

R: GCAGGCAGCGTATATCAGGAG

p53

F: CACAGCGTGGTGGTACCTTA

60

40

R: TCTTCTGTACGGCGGTCTCT

58

40

40

60

$\underline{\mathbf{T}_{\mathrm{m}}} \underline{\left({ }^{\circ} \mathrm{C}\right)}$ cycles $\underline{\text { ref }}$

129

112 


\begin{tabular}{|c|c|c|c|c|}
\hline \multirow{2}{*}{ Pai1 } & F: TCGTGGAACTGCCCTACCAG & \multirow[t]{2}{*}{60} & \multirow[t]{2}{*}{35} & \multirow[t]{2}{*}{130} \\
\hline & R: ATGTTGGTGAGGGCGGAGAG & & & \\
\hline \multirow[t]{2}{*}{ Slug } & F: CGCTCCTTCCTGGTCAAGA & 60 & 40 & $*$ \\
\hline & R:AGGTATAGGGTAACTTTCATAGAGATA & & & \\
\hline \multirow[t]{2}{*}{$\alpha \mathrm{SMA}$} & F: CCACCGCAAATGCTTCTAAGT & 58 & 40 & 136 \\
\hline & R: GGCAGGAATGATTTGGAAAGG & & & \\
\hline \multirow[t]{2}{*}{ Snail } & F: GTGCCCACCTCCAAACCC & 60 & 40 & $*$ \\
\hline & R: AAGGACATGCGGGAGAAGG & & & \\
\hline \multirow[t]{2}{*}{$\operatorname{Tgf} \beta$} & F: CAGTGGCTGAACCAAGGAGAC & 60 & 35 & 130 \\
\hline & R: ATCCCGTTGATTTCCACGTG & & & \\
\hline \multirow[t]{2}{*}{ Twist } & F: TGATAGAAGTCTGAACACTCGTTTG & 60 & 40 & $*$ \\
\hline & R: GGCTGATTGGCAAGACCTCT & & & \\
\hline \multirow[t]{2}{*}{ Vegf } & F: TTACTGCTGTACCTCCACC & 60 & 35 & 131 \\
\hline & R: ACAGGACGGCTTGAAGATG & & & \\
\hline \multirow[t]{2}{*}{ Vimentin } & F: CGGAAAGTGGAATCCTTGCA & 60 & 40 & 137 \\
\hline & R: CACATCGATCTGGACATGCTGT & & & \\
\hline
\end{tabular}

*Primer sequences were designed and purchased from Primerdesign Ltd. (Southhampton, UK). 


\subsection{Western Blot Analysis}

Frozen heart tissue was pulverized on liquid nitrogen and resuspended in protein lysis buffer. ${ }^{122}$ After incubation for 20 minutes on ice, heart lysates were cleared by centrifugation at 13,000 rpm for 10 minutes at $4^{\circ} \mathrm{C}$. Equal amounts of protein $(40 \mu \mathrm{g})$ were boiled for $5 \mathrm{~min}$ in $2 \mathrm{X}$ loading buffer and fractionated by SDS polyacrylamide gel electrophoresis together with molecular weight standards and transferred to nitrocellulose membranes. Membranes were blocked in 5\% bovine serum albumin/ $5 \%$ milk powder for 1-2 hours prior to incubation with primary antibodies overnight at $4^{\circ} \mathrm{C}$ with antibodies against p53, p21 or murine double minute (mdm)-2, Hif1 $\alpha$, Vegf or Gapdh. Protein bands were visualized using horseradish peroxidase-conjugated secondary antibodies (dilution, 1:3,000) for 2 hours, followed by detection with SuperSignal ${ }^{\circledR}$ West Pico Substrate. Protein bands were quantified by densitometry and normalized to GAPDH protein and are expressed as -fold change vs. shamoperated mice.

\subsection{Statistical Analysis}

Quantitative data are presented as mean \pm standard error of the mean (SEM). Differences were tested by Student's $t$ test for unpaired means or paired means, if findings in the same animal before and after an intervention were compared. If more than two groups were compared, the One Way Analysis of Variance (ANOVA) test was performed, followed by Bonferroni's Multiple Comparison test. Frequencies were compared using the $\chi^{2}$ test. Statistical significance was assumed if $\mathrm{P}$ reached a value $<0.05$. All analyses were performed using GraphPad PRISM data analysis software. 


\section{Results}

\subsection{Generation of Endothelial-Specific p53 Knockout Mice}

To generate endothelial-specific p53 knockout mice (End.p53-KO), mice carrying floxed p53 alleles $^{21}$ were crossed with mice expressing Cre recombinase under control of an inducible Tie2 promoter (Tie2.ERT.Cre). ${ }^{119}$ Gene deletion was achieved by feeding mice with tamoxifen-containing chow and confirmed by PCR analysis of genomic DNA from tail biopsies (Figure 5.1.A). Quantitative real time PCR analysis revealed that p53 mRNA levels were significantly reduced (to approximately 10\%) in CD31-positive cells isolated from hearts of End.p53-KO mice compared to CD31-positive cells isolated from End.p53-WT mice or CD31-negative cells (Figure 5.1.B).

A

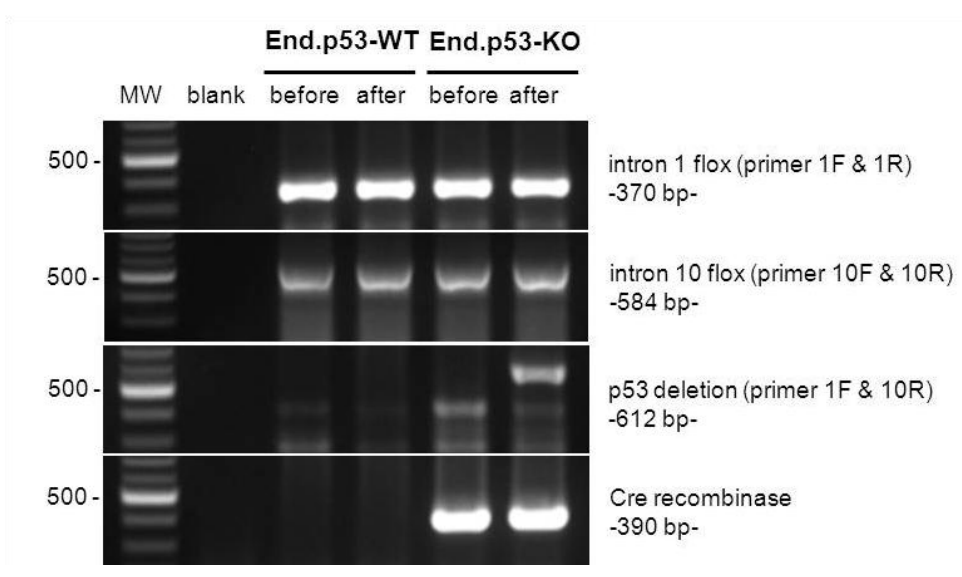

B

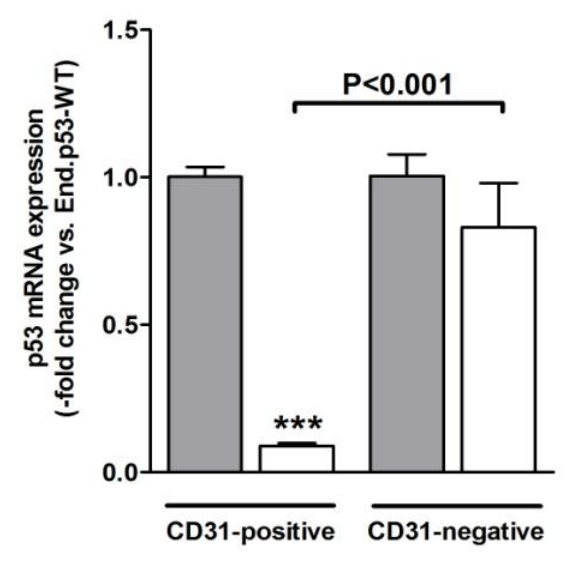

Figure 5.1. Genotyping of endothelial p53 knockout and wildtype mice. A, Genomic DNA was isolated from mouse tail biopsies obtained before and 6 weeks after feeding mice with tamoxifen citrate-containing chow and amplified by PCR. Floxed p53 alleles were identified using forward (F) and reverse (R) primer against intron 1 or intron 10. Excision of p53 exons was confirmed in Cre recombinase transgenic mice. MW, molecular weight marker. B, qPCR analysis of p53 mRNA expression in CD31-positive and CD31-negative cells, isolated from hearts of End.p53-WT (grey bars) and End.p53-KO (white bars) mice. ***P<0.001 vs. CD31-positive cells isolated from End.p53-WT mice. Significant differences between CD31-positive and CD31-negative cells from End.p53-KO mice are indicated within the graph. 
Flow cytometry showed $24.5 \%$ of Tie 2 expression in cardiac cells, but only 1.4 and $2.7 \%$ in blood and spleen mononuclear cells, respectively, and no CD45-positive cells were detected in the heart (Figure 5.2).

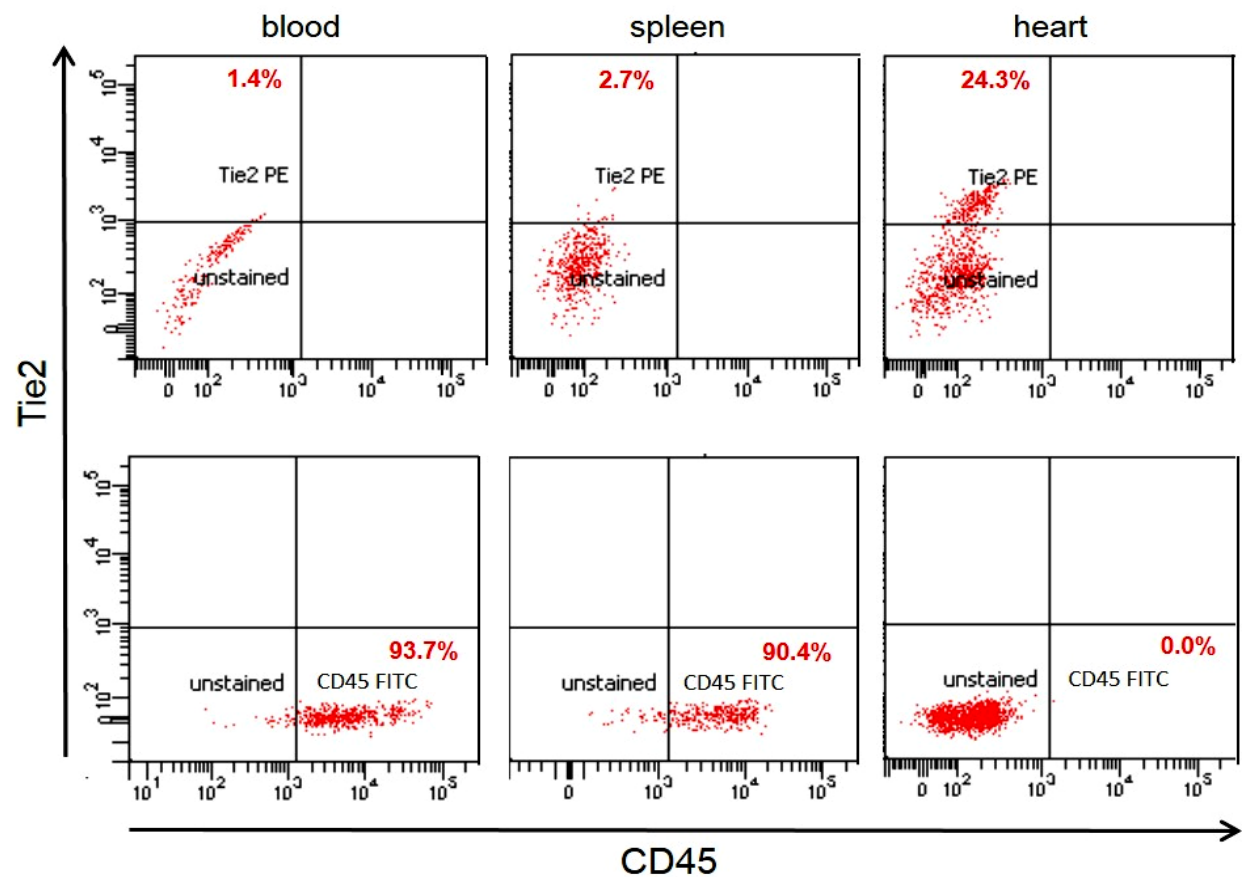

Figure 5.2. Flow cytometry of Tie2- and CD45-positive cells isolated from blood, spleen and heart of End.p53-WT mice.

Of note, EC-specific p53 deletion per se did not affect survival up to 12 months and was not associated with spontaneous tumor formation (not shown).

\subsection{Endothelial p53 Deletion Protects Against Cardiac Hypertrophy and Dysfunction After Pressure Overload}

To induce cardiac hypertrophy and heart failure, female mice were subjected to TAC surgery. Mean pressure gradients over the aortic valve were similar in both groups, i.e. $71 \pm 3.8 \mathrm{mmHg}$ in End.p53-KO and 74 $\pm 3.7 \mathrm{mmHg}$ in End.p53-WT mice $(\mathrm{P}=0.51)$ (Figure 5.3.A). As shown in Figure 5.3.B, survival (after exclusion of mice that died within the first 24 hours after surgery) was significantly improved in End.p53-KO mice (5 out of 34 mice died) compared to their End.p53-WT counterparts (16 out of 41 mice died; $\mathrm{P}=0.020$ ). Mice were not followed for more than 20 weeks to avoid prolonged suffering of heart failure symptoms. 
A

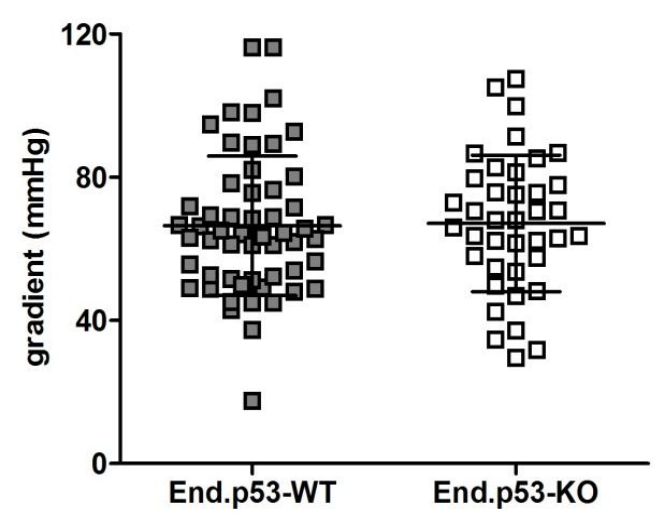

B

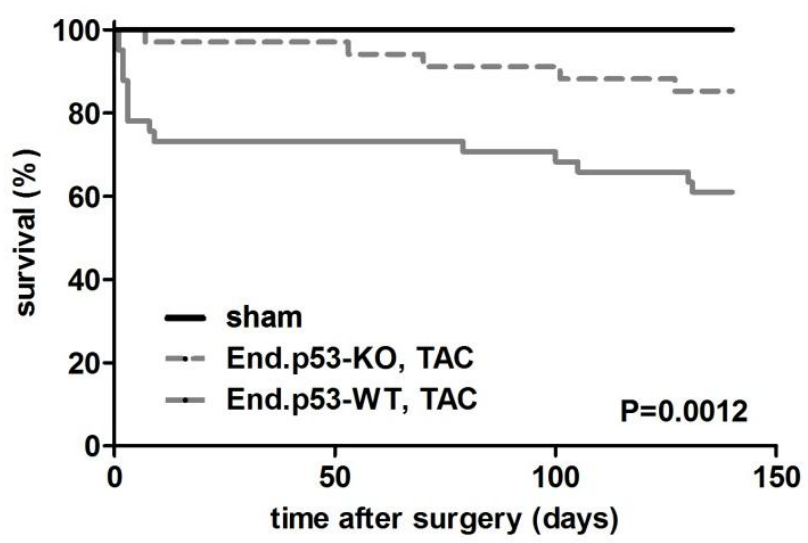

Figure 5.3. Effect of endothelial p53 deletion on survival. A, Mean pressure gradients over the aortic valve, i.e. $71 \pm 3.8 \mathrm{mmHg}$ in End.p53-KO and $74 \pm 3.7 \mathrm{mmHg}$ in End.p53-WT mice $(\mathrm{P}=0.51)$. B, Kaplan-Meier survival analysis of sham- $(\mathrm{n}=19)$ or TAC-operated End.p53-WT ( $\mathrm{n}=41)$ and End.p53-KO mice $(\mathrm{n}=34)$.

Serial echocardiographic measurements (representative $M$ mode recordings are shown in Figure 5.4.A) revealed a significant decline in cardiac function in End.p53-WT mice, beginning at week 4 after TAC and progressing towards week 20 compared to End.p53-KO mice. Fractional shortening (FS) was significantly reduced in End.p53-WT mice compared to End.p53-KO mice at 8 and 20 weeks after TAC (Figure 5.4.B). Compared to baseline, a significant increase in the endsystolic (Figure 5.4.C) and enddiastolic (Figure 5.4.D) LV inner diameter was observed in both the genotypes, which progressed further only in End.p53-WT mice and was significantly more pronounced compared to End.p53-KO mice at 20 weeks after TAC. At this time point, both the mean PWTh $(\mathrm{P}<0.05$; Figure 5.4.E) and echocardiographically determined LV weight $(\mathrm{P}<0.05$; Figure 5.4.F) were significantly increased in End.p53-WT compared to End.p53-KO mice, and this observation was further confirmed by determining the actual heart weight at necropsy (Figure 5.5.A) after 8 and 20 weeks time point. 
A

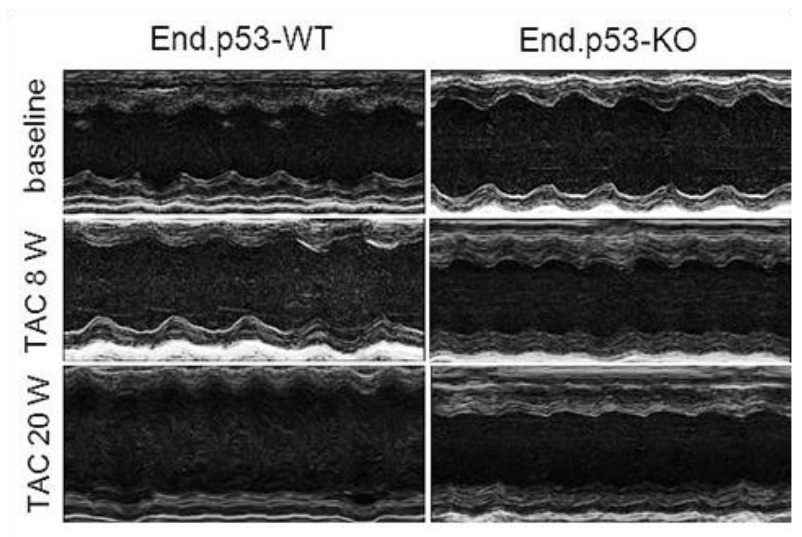

C

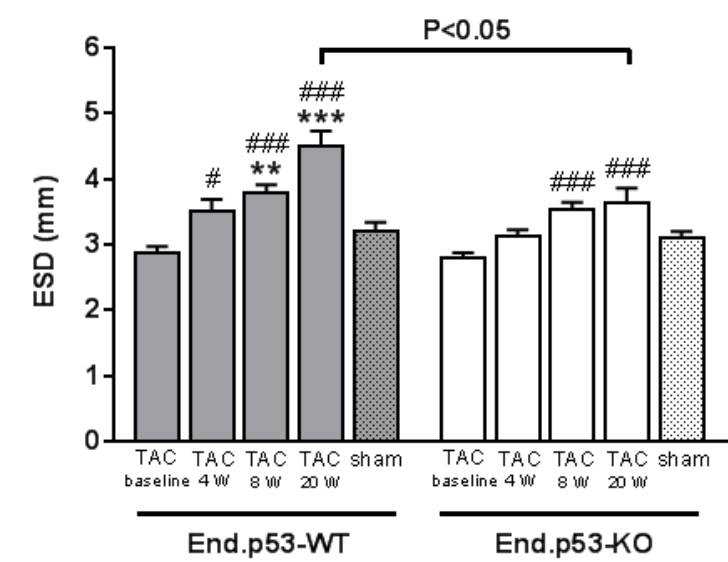

$\mathbf{E}$

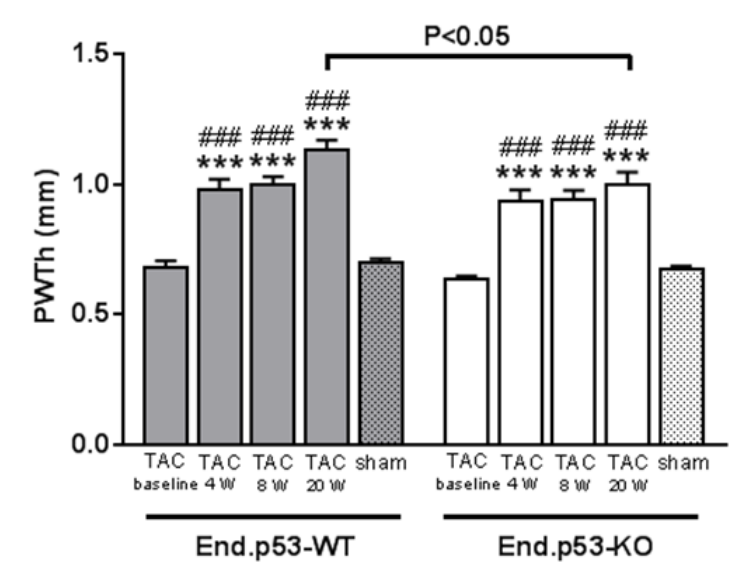

B

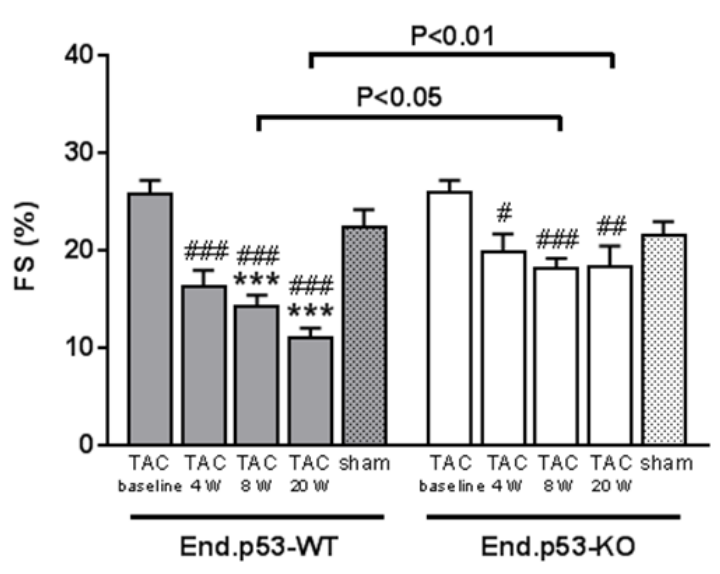

D

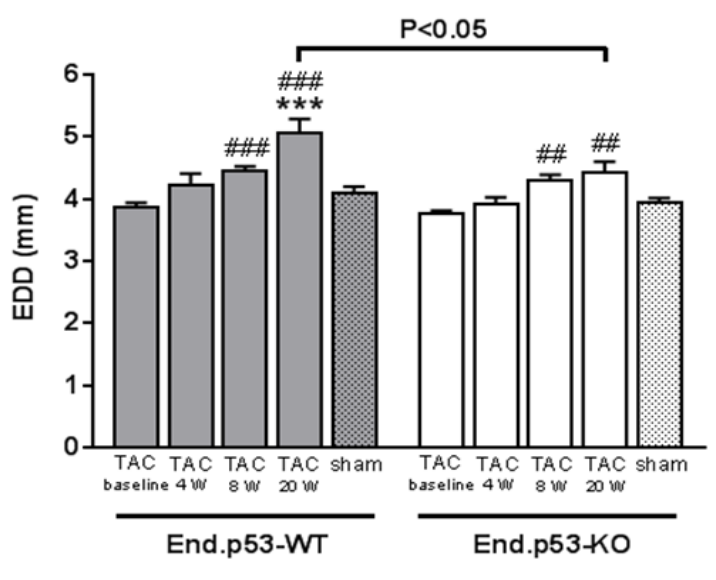

$\mathbf{F}$

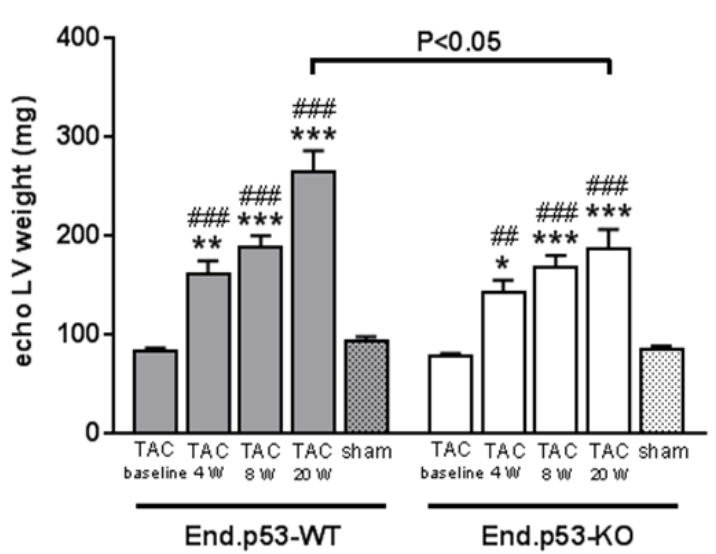

Figure 5.4 Effect of endothelial p53 deletion on cardiac hypertrophy and function. A, Representative Mmode tracings before (baseline) as well as 8 and 20 weeks after TAC. Mean values for fractional shortening (FS; $\mathbf{B})$, endsystolic (ESD; C) and enddiastolic (EDD; D) inner LV diameters, posterior wall thickness (PWTh; E), LV weight determined by echocardiography(F) Grey bars: End.p53-WT mice; white bars: End.p53-KO mice; dotted bars: sham-operated mice. ${ }^{*} \mathrm{P}<0.05$, $* * \mathrm{P}<0.01$ and $* * * \mathrm{P}<0.001$ vs. sham, \#P<0.05, \#\#P<0.01 and \#\#\#<<0.001 vs. baseline. Significant differences between End.p53-WT and End.p53-KO mice are indicated within the graphs. 
Cardiac hypertrophy was also determined in the histological level, the mean cardiomyocyte cross-sectional area (CSA; Figure 5.5. C and B) was significantly increased after TAC in both genotypes, although to a lesser extent in End.p53-KO mice ( $\mathrm{P}<0.05$ at 20 weeks after TAC), suggesting that inducible deletion of p53 specifically in endothelial cells ameliorates the development of cardiac hypertrophy and the deterioration of cardiac function after pressure overload.

A

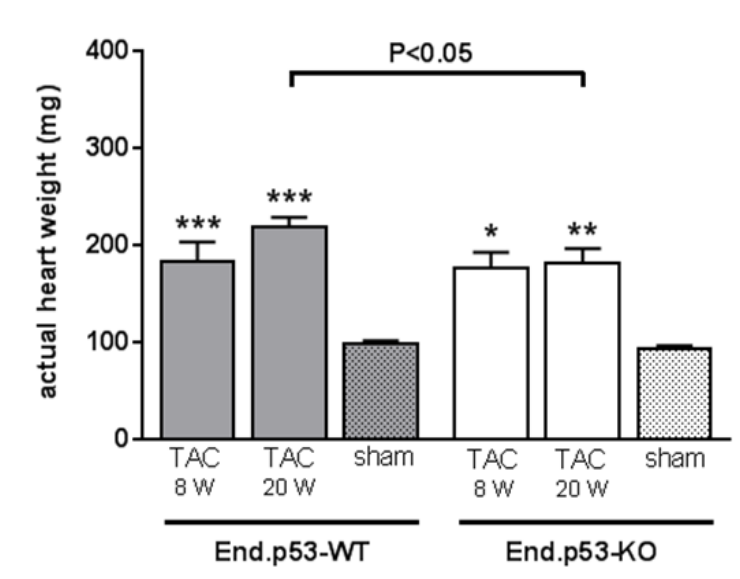

B

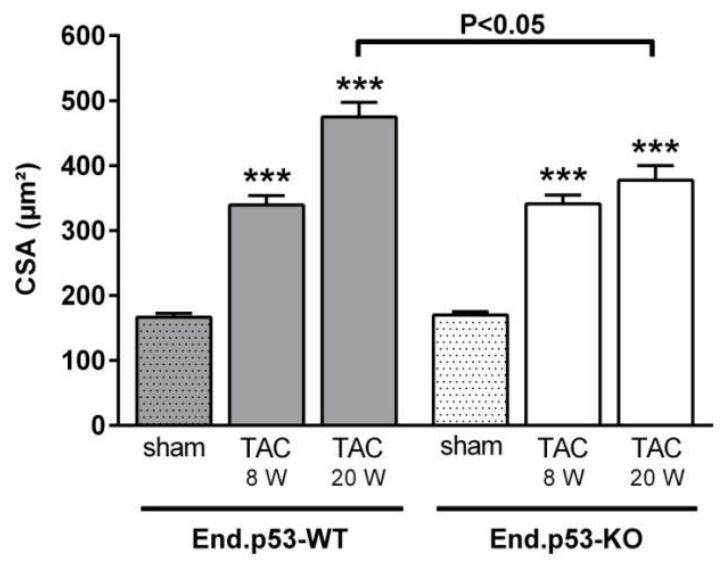

C
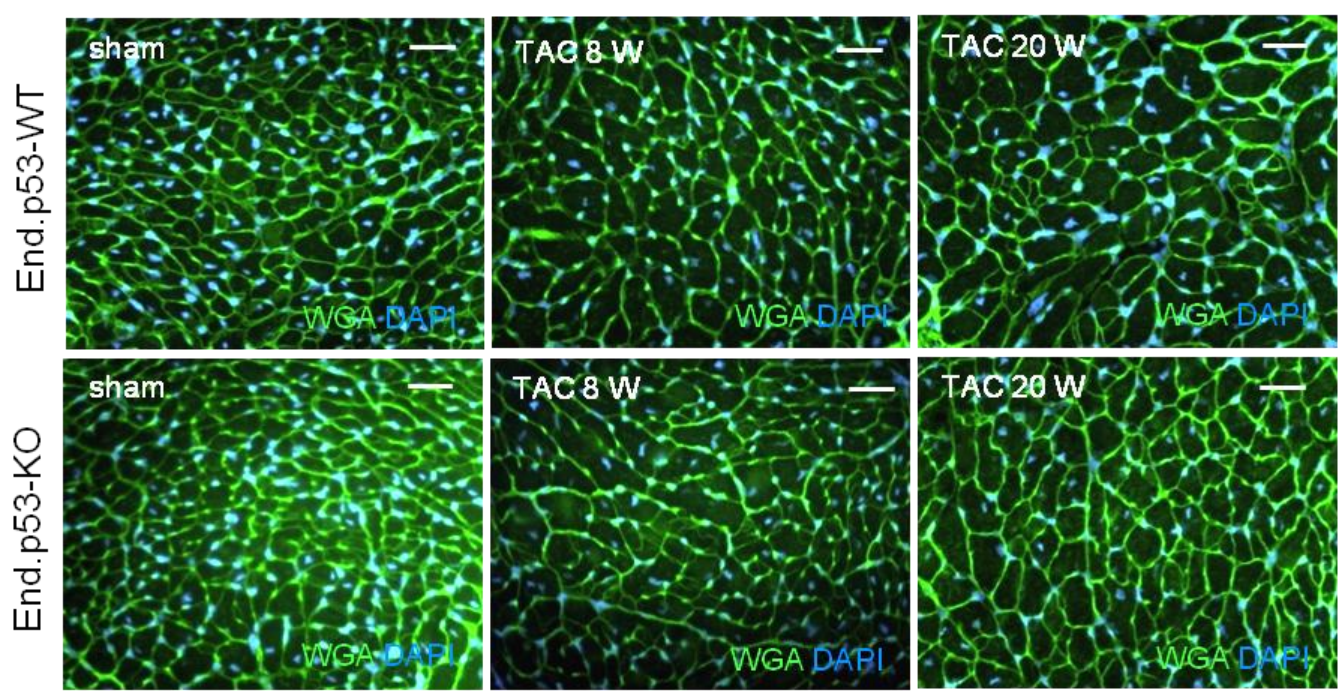

Figure 5.5. Effect of endothelial p53 deletion on cardiac hypertrophy. Heart weight determined at necropsy, at baseline and different time points after TAC or sham operation are shown. $\mathbf{A}$ and $\mathbf{B}$, results after quantification of the cardiomyocyte cross-sectional area (CSA; n=7-16 mice per group) and $\mathbf{C}$, representative pictures of WGA-stained myocardial cross sections. Size bars represent $100 \mu \mathrm{m}$. Grey bars: End.p53-WT mice; white bars: End.p53-KO mice; dotted bars: sham-operated mice. ${ }^{*} \mathrm{P}<0.05$, $* * \mathrm{P}<0.01$ and ${ }^{*} * * \mathrm{P}<0.001$ vs. sham, $\# \mathrm{P}<0.05, \# \# \mathrm{P}<0.01$ and \#\#\#P<0.001 vs. baseline. Significant differences between End.p53-WT and End.p53KO mice are indicated within the graphs. 


\subsection{Endothelial p53 Deletion Reduces Cardiac p53 Accumulation in Endothelial and Non-Endothelial Cells after TAC}

Cardiac hypertrophy is associated with increased apoptosis and elevated cardiac p53 levels. ${ }^{80,81}$ Our analyses could confirm these previous findings in End.p53-WT mice, whereas cardiac p53 mRNA (Figure 5.6.A) and protein (Figure 5.7.A and D) levels were not altered in hearts of End.p53-KO and significantly lower than in End.p53-WT mice, particularly 8 weeks after TAC. Similar findings were observed for cardiac mRNA and protein expression levels of p21 (Figure 5.6.B, 5.7.B and D), a cyclin-dependent kinase inhibitor and downstream target of $\mathrm{p} 53$. On the other hand, cardiac levels of $\mathrm{mdm} 2$, a negative regulator of p53-mediated transactivation, were not significantly altered at any of the time points examined (Figure 5.6.C, 5.7.C and D).

A

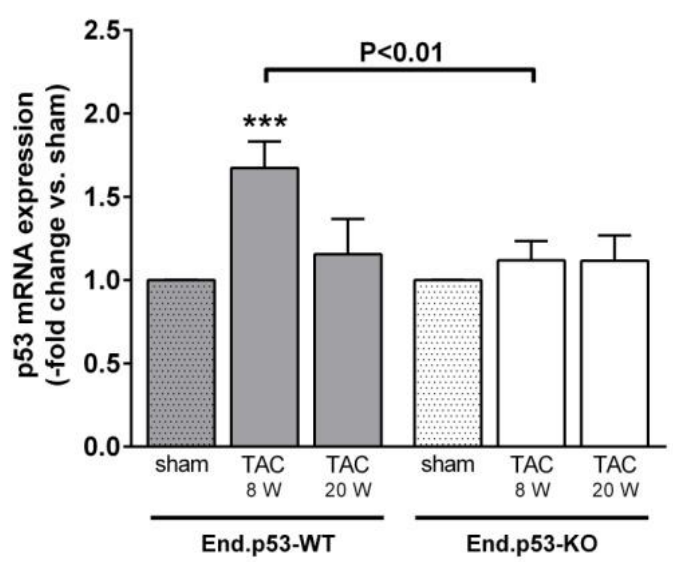

C

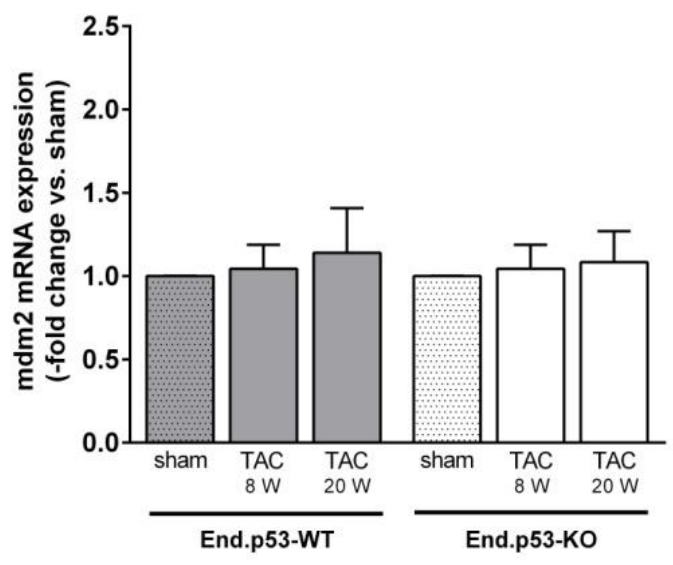

B

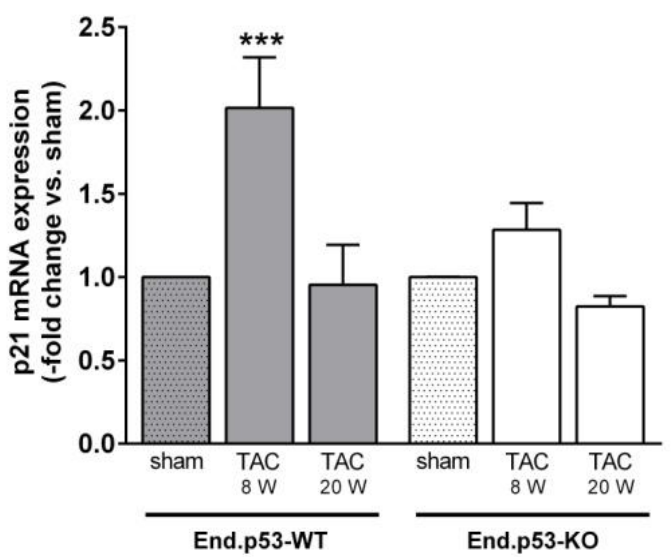

Figure 5.6. mRNA expression of $\mathrm{p} 53$ and p53regulated factors in banded mouse hearts. The relative cardiac mRNA expression levels of p53, p21 and $\mathrm{mdm} 2$ were analyzed using quantitative real time PCR (A-C) Results were normalized to Gapdh and expressed as -fold change vs. shamoperated littermates of the same genotype (set at 1 ; dotted bars). $\quad * \mathrm{P}<0.05, \quad * * \mathrm{P}<0.01 \quad$ and $* * * \mathrm{P}<0.001$ vs. sham. Significant differences between End.p53-WT and End.p53-KO mice are indicated within the graphs. 
A

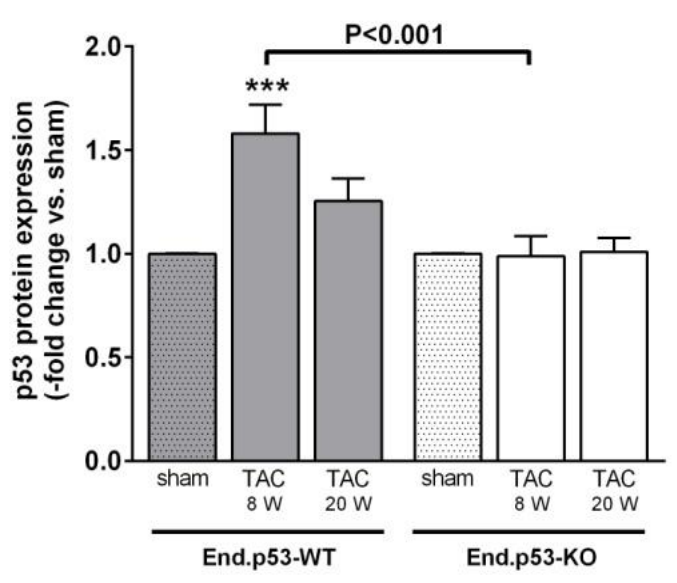

C

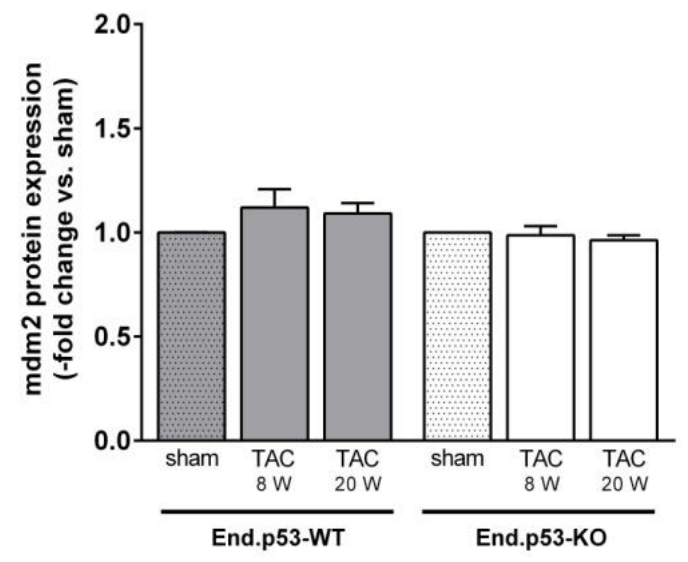

B

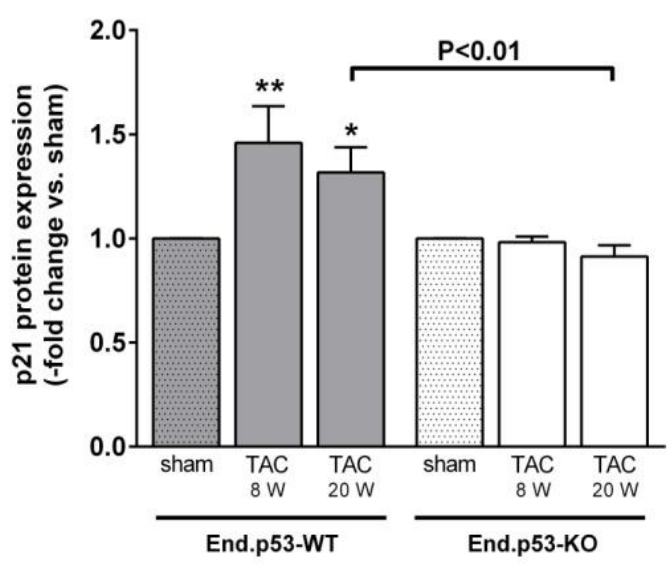

D

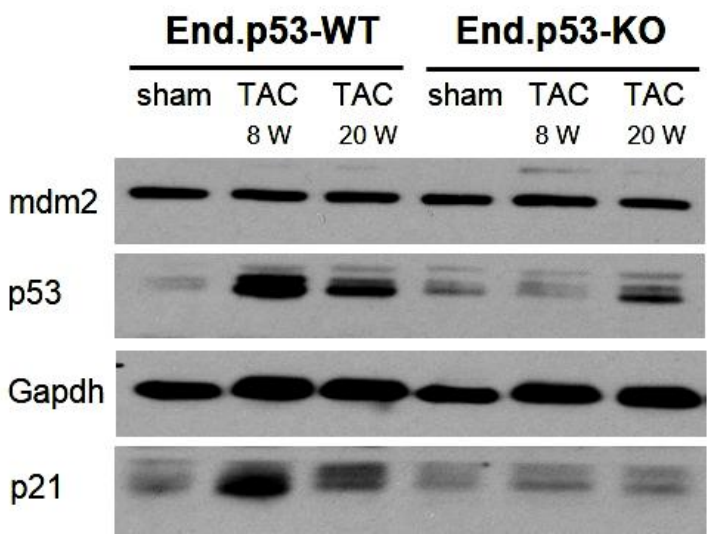

Figure 5.7. Protein expression of p53 and p53-regulated factors in banded mouse hearts. The relative cardiac protein expression levels of p53, p21 and mdm2 were analyzed using Western blot (A-C) in End.p53WT (grey bars) and End.p53-KO mice (white bars) (n=8-19 mice per group). Representative Western blot findings are shown in D. Results were normalized to Gapdh and expressed as -fold change vs. sham-operated littermates of the same genotype (set at 1 ; dotted bars). $* \mathrm{P}<0.05$, $* * \mathrm{P}<0.01$ and $* * * \mathrm{P}<0.001$ vs. sham. Significant differences between End.p53-WT and End.p53-KO mice are indicated within the graphs.

After the previous result, showing that there is reduction in p53 mRNA and protein levels in End.p53-KO mice after TAC, we hypothesized that endothelial p53 deletion might reduce the apoptosis in the hearts after TAC in End.p53-KO mice. To test this hypothesis, we first stained the heart sections of sham, End.p53-WT and End.p53-KO at 8 and 20 weeks after TAC for p53. Interestingly, fluorescence microscopy images (Figure 5.8.A-C) shown not only reduced numbers of p53 positive endothelial cells, but also reduced numbers of 
apoptotic non-endothelial cells in End.p53-KO mice compared to End.p53-WT mice. Sham mice expressed very low basal levels of p53.

A
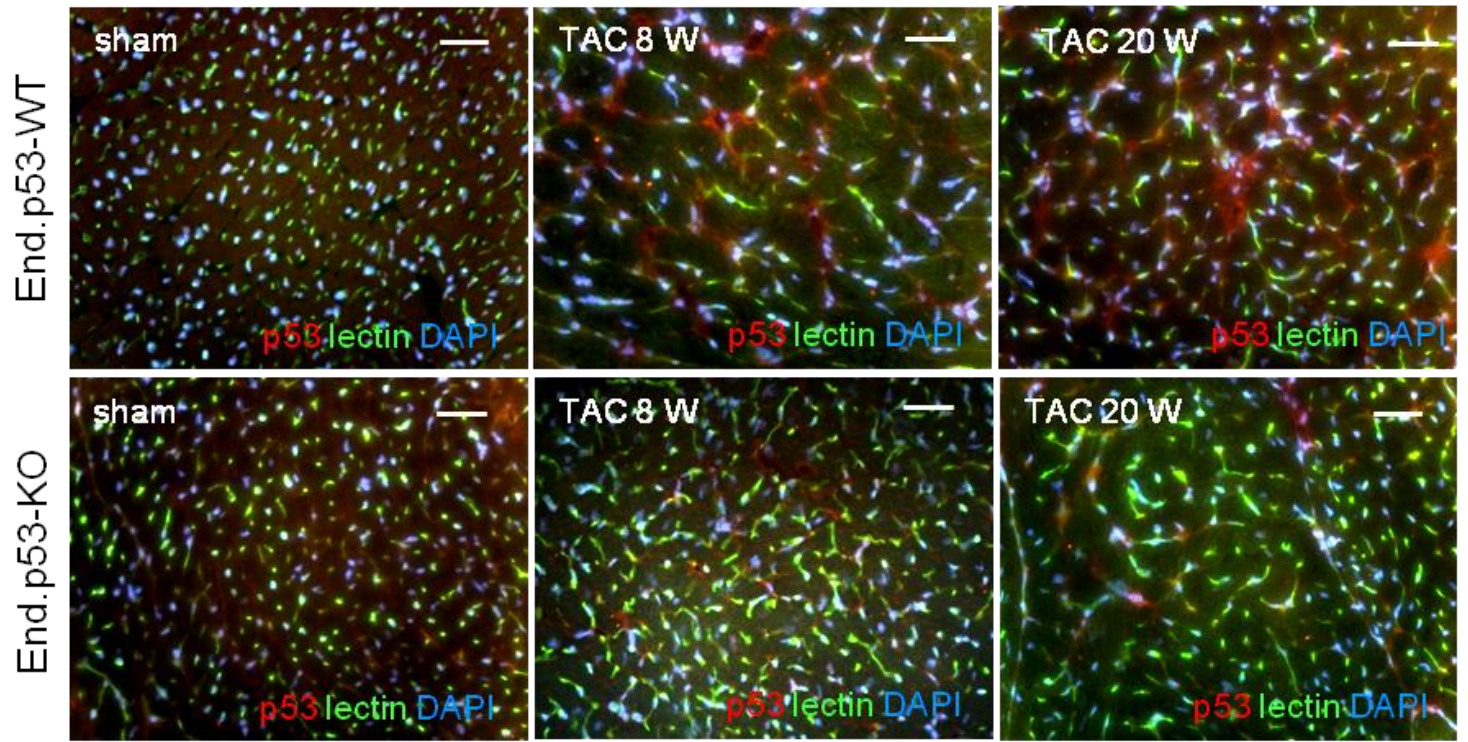

B

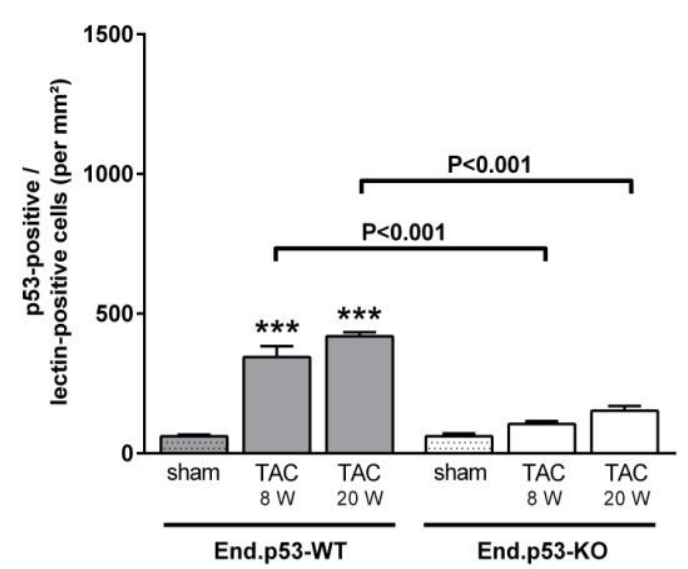

C

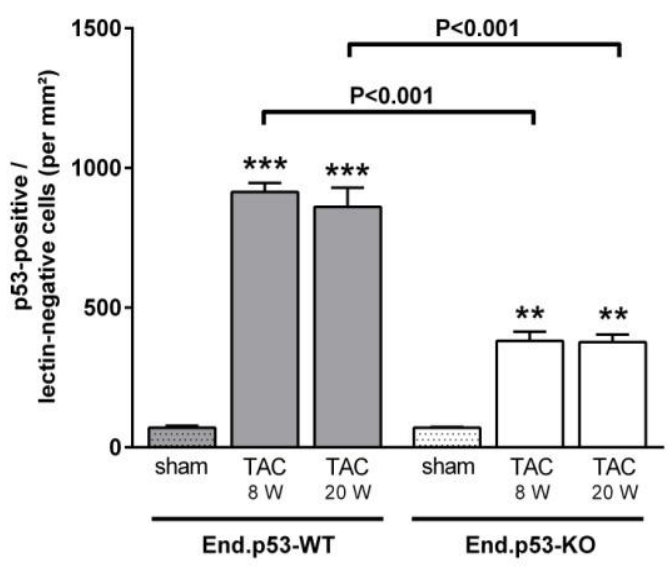

Figure 5.8. Reduced number of p53 positive endothelial and non endothelial cells in End.p53-KO mice. Cardiac cross sections were examined for the expression of p53 (A-C). Representative pictures of findings in End.p53-WT and End.p53-KO mice after TAC are shown in A. Intravitally isolectin B4 perfused capillaries are green, DAPI-positive cell nuclei blue, p53 in red. Size bars represent $100 \mu \mathrm{m}$. The summary of findings in $\mathrm{n}=3$ 6 mice per group is shown in $\mathbf{B}, \mathbf{C} .{ }^{*} * \mathrm{P}<0.01 * * * \mathrm{P}<0.001$ vs. sham. Differences between End.p53-WT and End.p53-KO mice are indicated within the graphs. 


\subsection{Endothelial p53 Deletion Reduces Apoptosis in Endothelial and Non- Endothelial Cells After TAC}

To assess the effect of endothelial cell p53 deletion in apoptosis after TAC, we stained the tissues of sham, End.p53-WT and End.p53-KO after 8 and 20 weeks after TAC with apoptotic markers such as cleaved caspase 3 and TUNEL. Fluorescence microscopy confirmed not only reduced numbers of lectin-positive (endothelial) cells, but also lectinnegative (non endothelial) cells, double-immunopositive for activated caspase-3 (Figure 5.9.A-C) or TUNEL (Figure 5.10.A-C) in hearts of End.p53-KO compared to End.p53-WT mice.

A
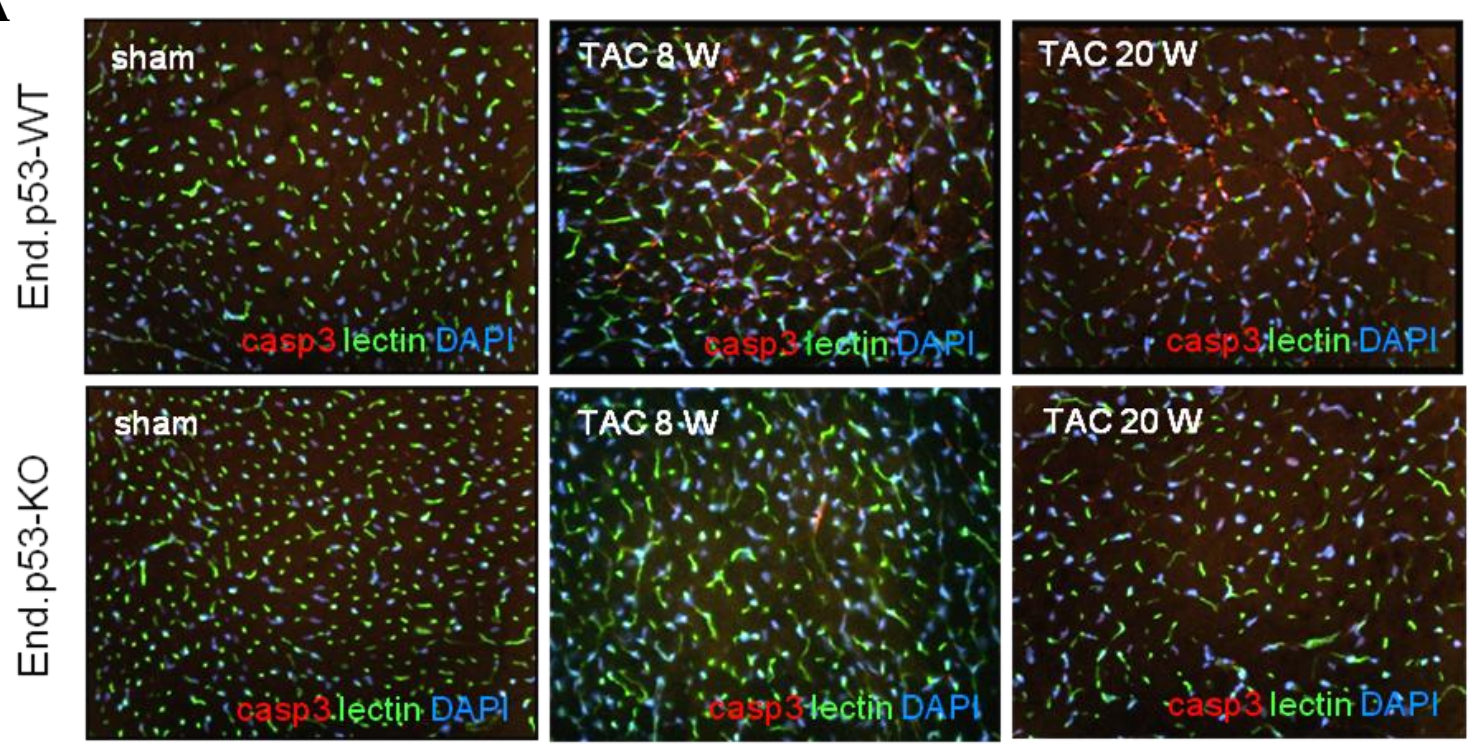

B

C
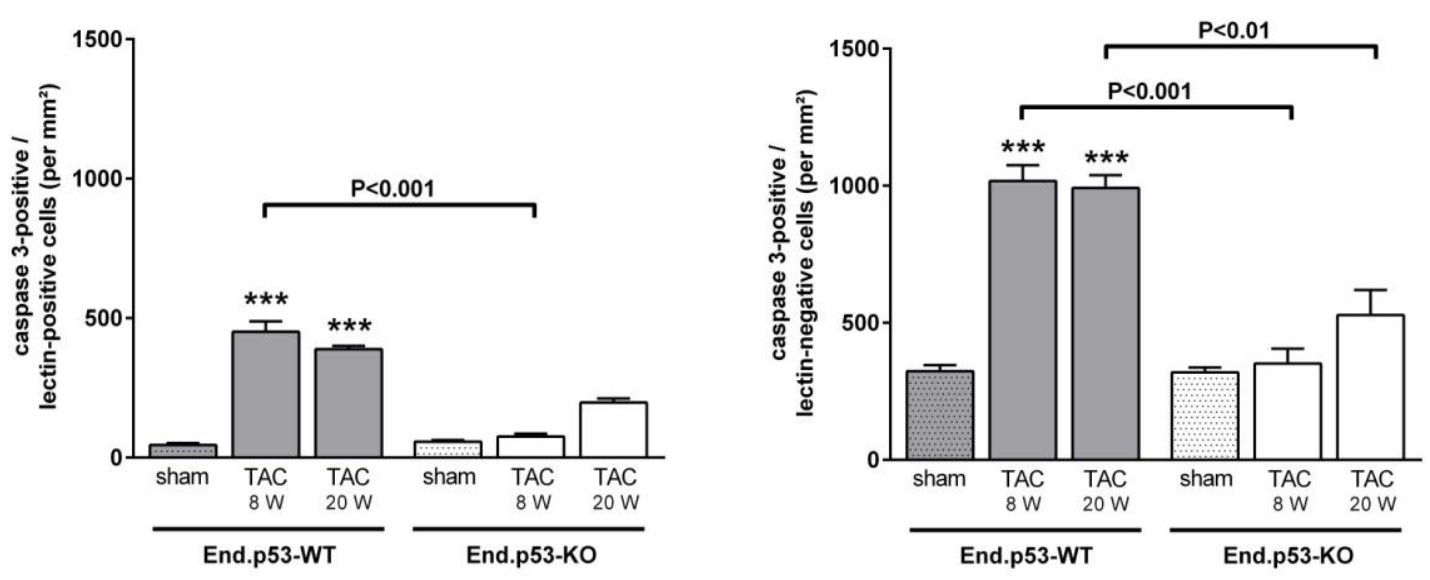

Figure 5.9. Effects of endothelial p53 deletion on caspase- 3 levels after TAC. Cardiac cross sections through the LV were examined for the expression of activated caspase-3 shown in red, Intravitally isolectin B4 
perfused capillaries are green, DAPI-positive cell nuclei blue. Representative pictures of findings in End.p53WT and End.p53-KO mice after sham or TAC surgery (8 and 20 weeks) are shown in A. Size bars represent 100 $\mu \mathrm{m}$. The summary of findings in $\mathrm{n}=3-6$ mice per group is shown in $\mathbf{B}$ and $\mathbf{C} * \mathrm{P}<0.05$, $* * \mathrm{P}<0.01$ and $* * * \mathrm{P}<0.001$ vs. sham. Significant differences between End.p53-WT and End.p53-KO mice are indicated within the graphs.

A

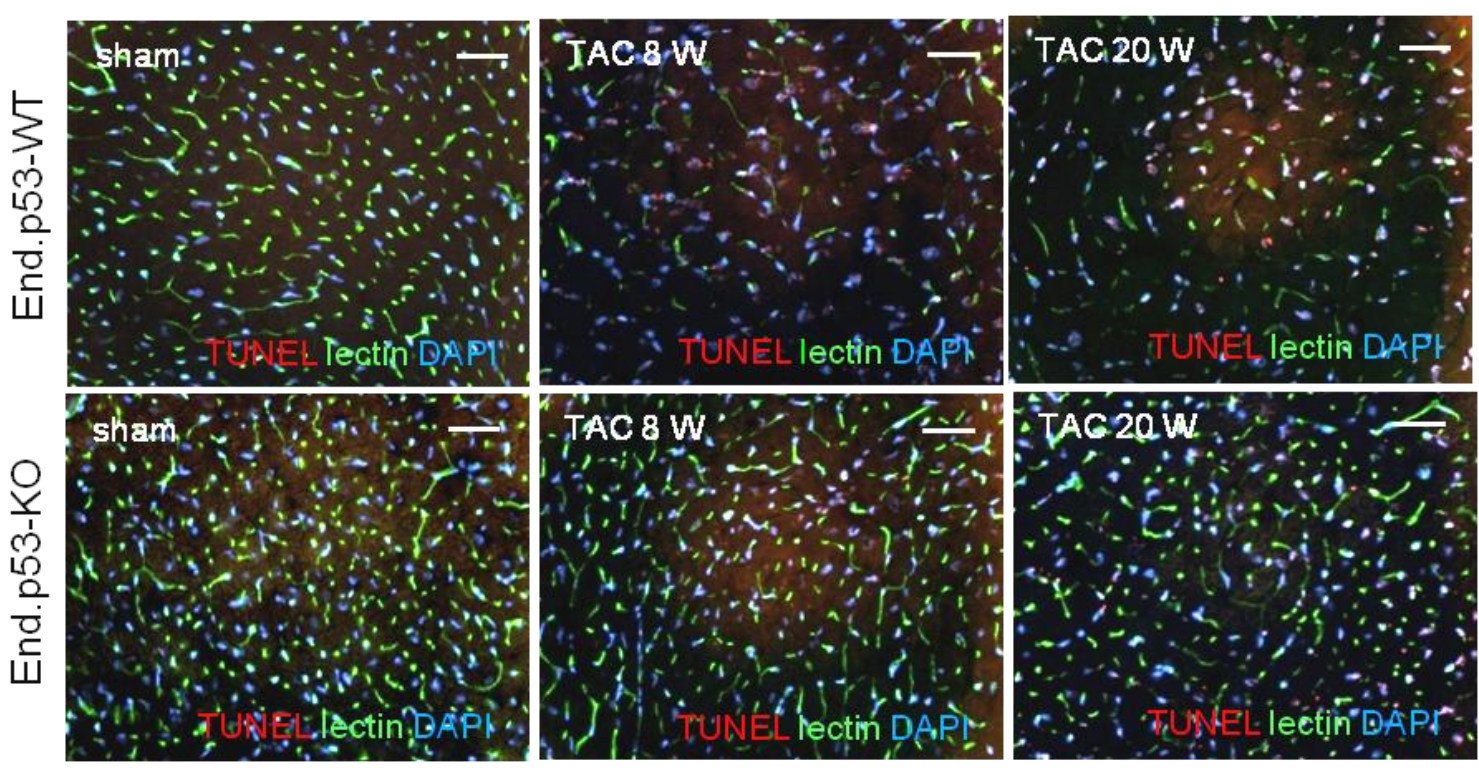

B

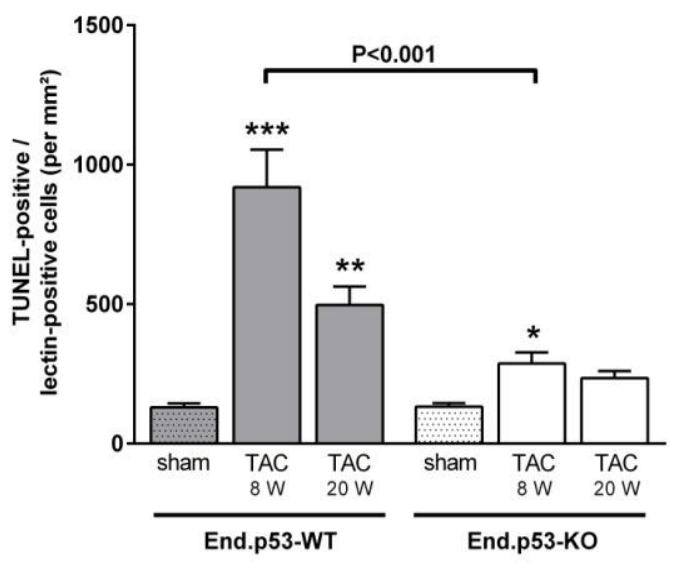

C

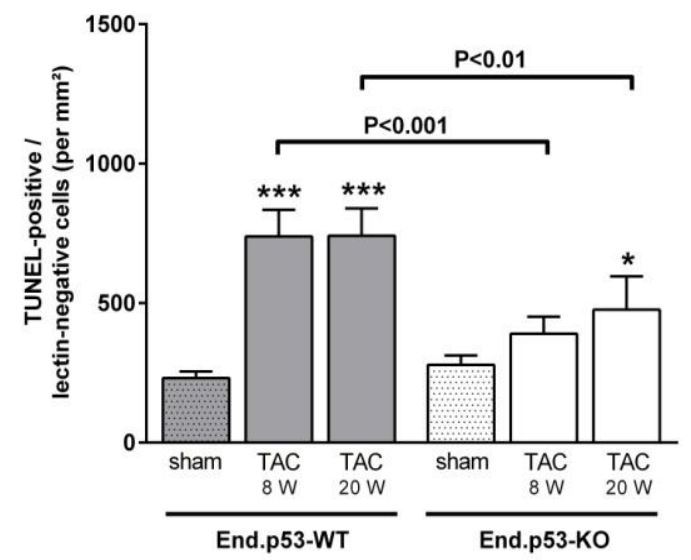

Figure 5.10. Effects of endothelial p53 deletion on cardiac apoptosis after TAC. Cardiac cross sections through the LV were examined for the expression of terminal deoxynucleotidyl transferase dUTP nick end labeling (TUNEL)-positive cells are red, Intravitally isolectin B4 perfused capillaries are green, DAPI-positive cell nuclei blue. Representative pictures of findings in End.p53-WT and End.p53-KO mice after sham or TAC surgery ( 8 and 20 weeks) are shown in A. Size bars represent $100 \mu \mathrm{m}$. The summary of findings in $\mathrm{n}=3-6 \mathrm{mice}$ per group is shown in $\mathbf{B}$ and $\mathbf{C}$. $* \mathrm{P}<0.05$, $* * \mathrm{P}<0.01$ and $* * * \mathrm{P}<0.001$ vs. sham. Significant differences between End.p53-WT and End.p53-KO mice are indicated within the graphs. 


\subsection{Deletion of p53 in Endothelial Cells is Associated with Higher Cardiac Hif1 $\alpha$ and Vegf Expression}

Accumulation of p53 in the heart has been shown to be involved in Hifl $\alpha$ degradation, angiogenesis inhibition and cardiac dysfunction following pressure overload. ${ }^{87}$ To study the importance of endothelial p53 for Hifl $\alpha$ suppression, we examined Hifl $\alpha$ and Vegf protein levels in pressure-overloaded hearts of End.p53-WT and End.p53-KO mice. At 8 and 20 weeks after TAC, i.e. the time points examined throughout this study, Hifl $\alpha$ (Figure 5.11.A and B) and Vegf (Figure 5.11A and C) protein expression was found not to be altered in End.p53-WT mice, whereas Hif1 $\alpha$ and Vegf protein levels were slightly, but significantly elevated in End.p53-KO mice at 20 and 8 weeks after surgery, respectively.

$\mathbf{A}$

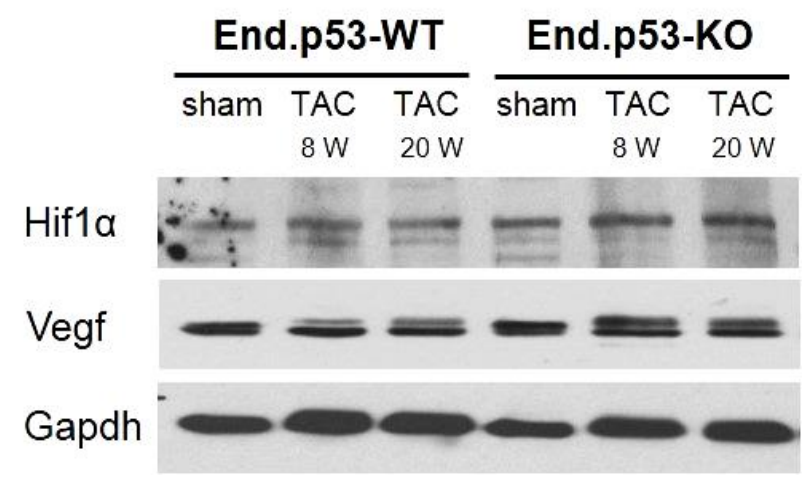

B

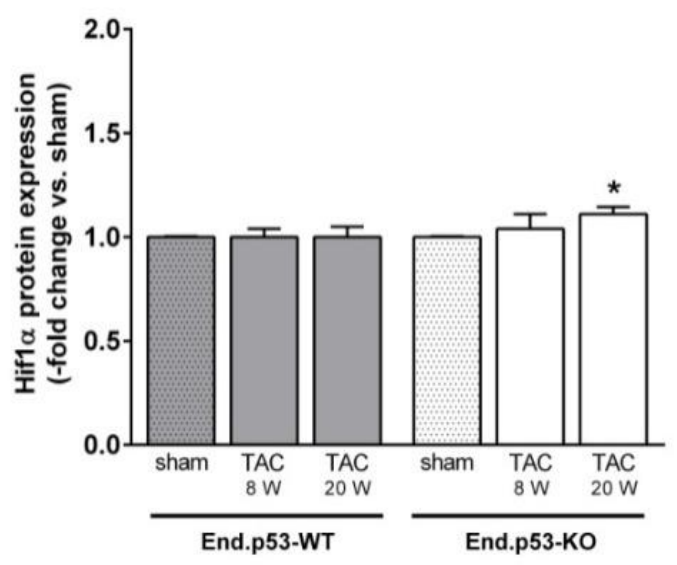

C

Figure 5.11. Role of endothelial p53 for the cardiac and endothelial response to hypoxia at 8 and 20 weeks after TAC. A-C, Western blot analysis of cardiac Hif1 $\alpha$ and Vegf protein levels in End.p53-WT and End.p53KO mice at different time points after transverse aortic constriction (TAC) or sham operation. Representative membranes are shown in $\mathbf{A}$ ( 8 and 20 weeks after TAC) Representative findings of the quantitative analysis of 
$\mathrm{n}=3$ independent experiments for Hif1 $\alpha$ and Vegf protein levels in $\mathbf{B}$ and $\mathbf{C}$. $* \mathrm{P}<0.05$ vs. sham. Significant differences between End.p53-WT and End.p53-KO mice are indicated within the graphs.

As we did not observe a significant difference in Hif1 $\alpha$ and Vegf protein levels at 8 and 20 weeks time point, we analyzed mouse hearts at earlier time points (i.e. at 7 days after TAC). Hypertrophic hearts after $7 \mathrm{~d}$ TAC revealed elevated Hifl $\alpha$ protein levels in both genotypes and confirmed that the TAC-induced increase in Hif1 $\alpha$ and Vegf expression was significantly more pronounced in mice lacking p53 in endothelial cells compared to their End.p53-WT counterparts (Figure 5.12. A-C).

A

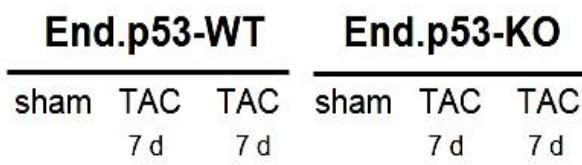

Hif1 $\alpha$

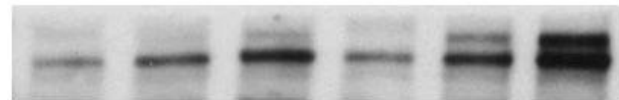

Vegf

Gapdh

B

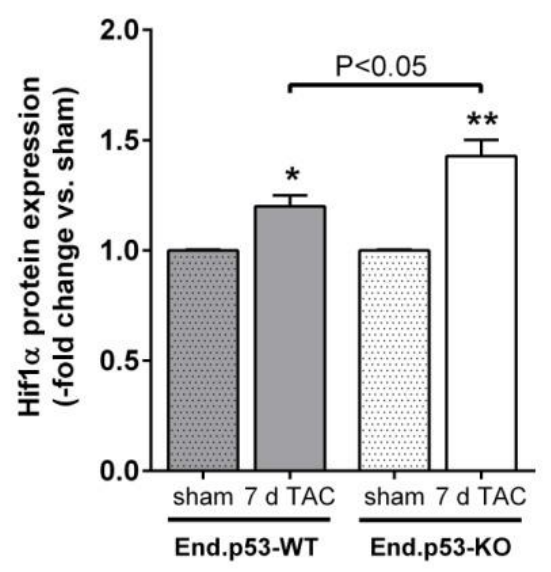

C

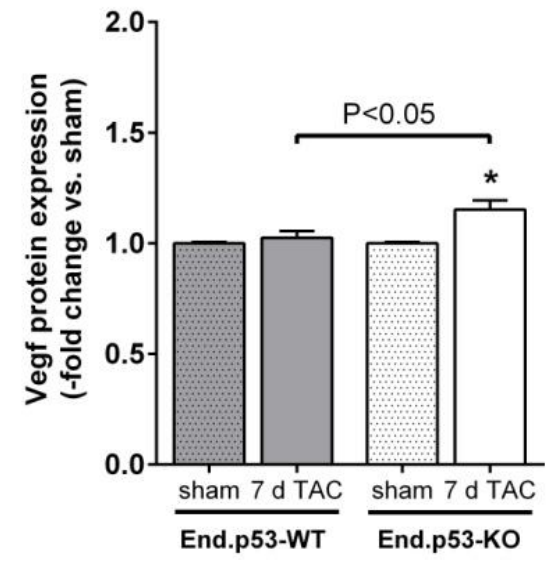

Figure 5.12. Role of endothelial p53 for the cardiac and endothelial response to hypoxia after 7 days TAC. Western blot analysis of cardiac Hif1 $\alpha$ and Vegf protein levels in End.p53-WT and End.p53-KO mice at 7 days time points after transverse aortic constriction (TAC) or sham operation. Representative membranes are shown in A. Representative findings of the quantitative analysis of $n=3$ independent experiments for Hif1 $\alpha$ and Vegf protein levels in $\mathbf{B}$ and $\mathbf{C}$. $* \mathrm{P}<0.05$ and $* * \mathrm{P}<0.01$ vs. sham. 
Analysis of HCMECs, stable transduced with lentiviral vectors containing either scr or p53 shRNA, followed by the exposure to $\mathrm{CoCl}_{2}$ confirmed that deletion of p53 in endothelial cells significantly enhanced Hif1 $\alpha$ protein levels in response to (chemical) hypoxia (Figure 5.13. $\mathrm{A}$ and $\mathrm{B})$.

A

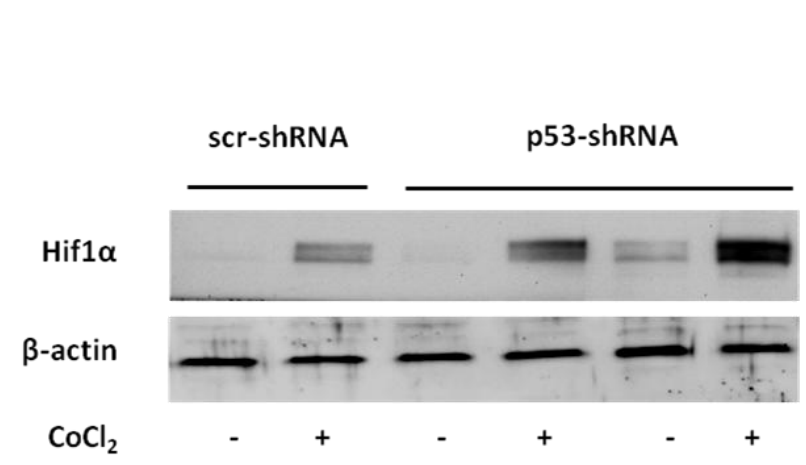

B

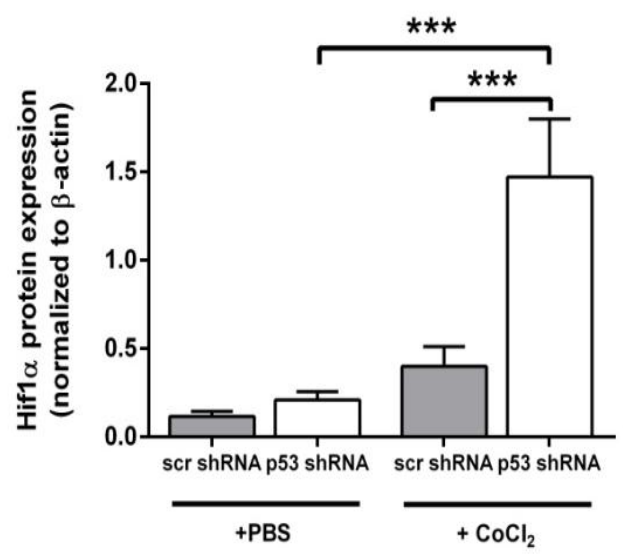

Figure 5.13. Deletion of p53 in endothelial cells induces HIF1 $\alpha$ expression when exposure to chemical hypoxia . A and B, Analysis of Hif1 $\alpha$ protein levels in HCMECs after stable transfection with a lentiviral p53 shRNA vector or scr shRNA (negative control) and exposure to chemical hypoxia ( $1 \mathrm{mM} \mathrm{CoCl}_{2}$ for 4 hours). Representative findings are shown in $\mathbf{A}$, the results of the quantitative analysis of $n=3$ independent experiments in $\mathbf{B} . * * * \mathrm{P}<0.001$.

\subsection{Deletion of p53 in Endothelial Cells Promotes Cardiac Angiogenesis}

To determine whether deletion of p53 and prevention of apoptotic cell death in endothelial cells is able to stabilize the cardiac microvasculature during experimental hypertrophy, the number of CD31-positive endothelial cells (Figure 5.14. A and B) and endothelial lectinperfused blood vessels (Figure 5.14. A and C) were quantified revealing a progressive decrease of functional (i.e. perfused) capillaries in hearts from End.p53-WT mice after TAC, whereas cardiac vascularization was significantly better in End.p53-KO vs. End.p53-WT mice 8 weeks and similar to sham-operated littermates 20 weeks after TAC. 
A
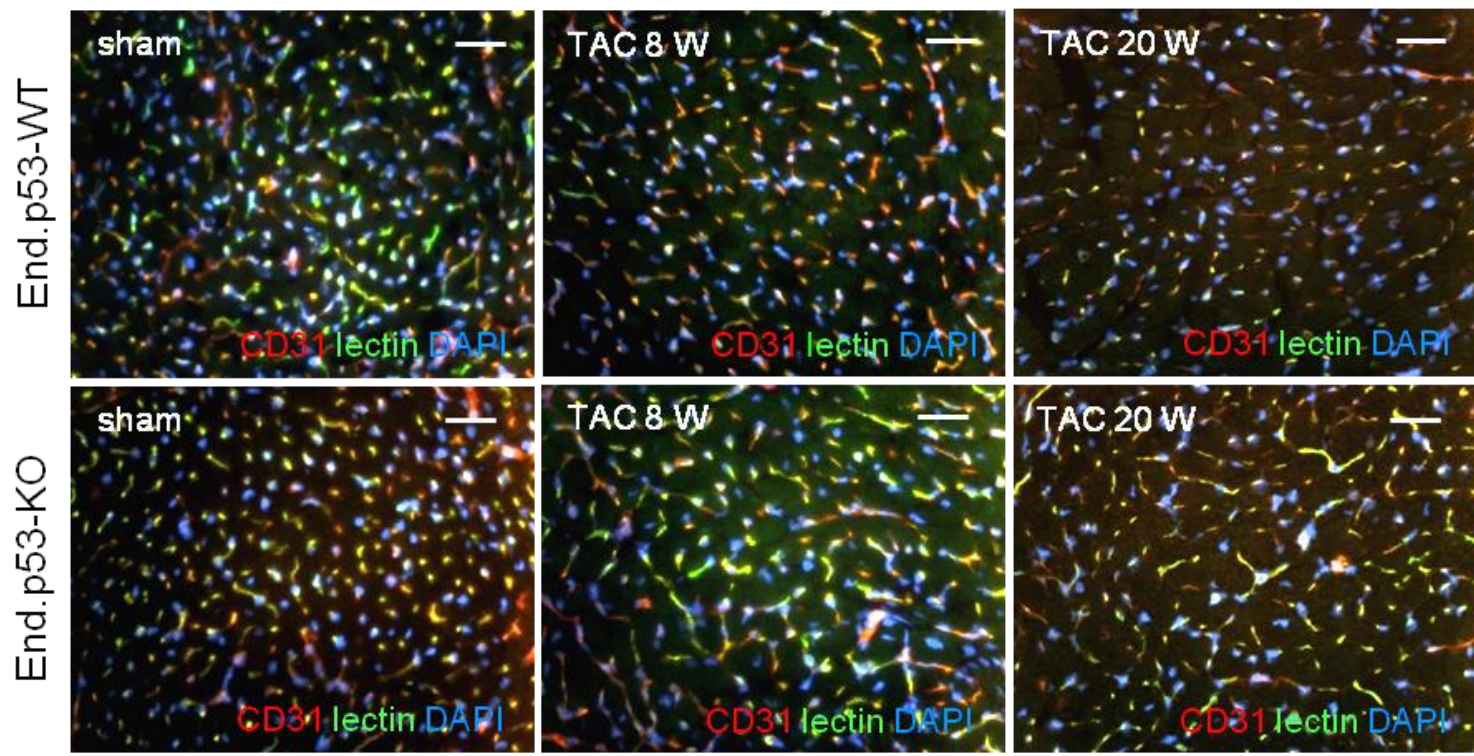

B

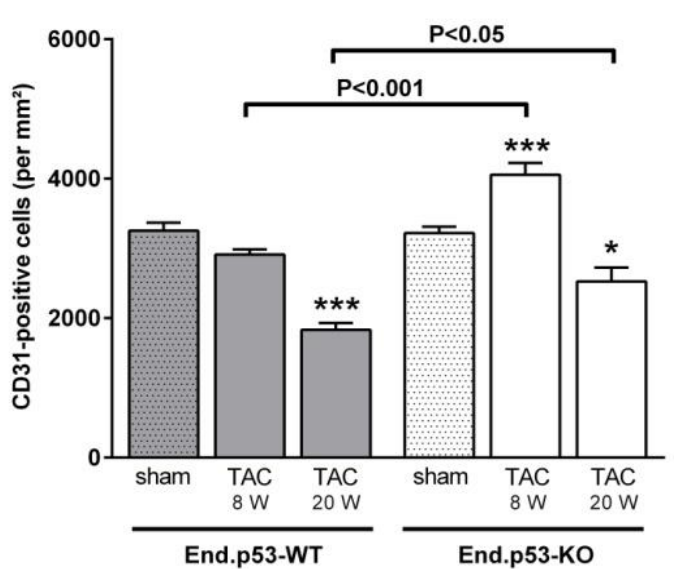

C

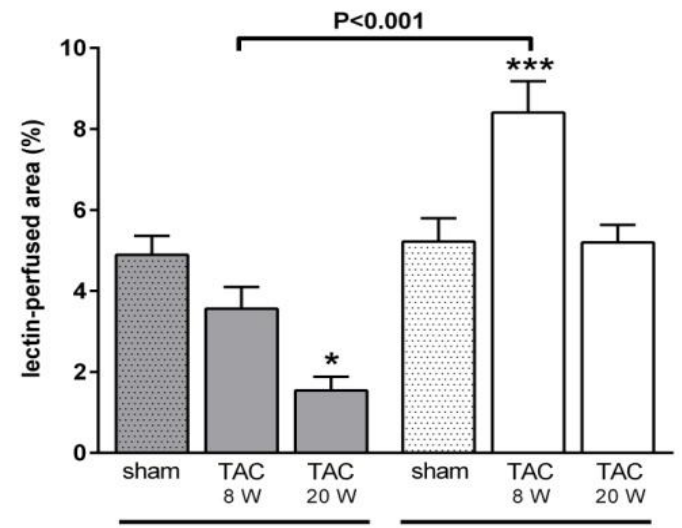

End.p53-WT

End.p53-KO

Figure 5.14. Cardiac vascularization in End.p53-WT and End.p53-KO mice. A, Representative pictures of CD31-immunopositive, isolectin B4-perfused capillaries in hearts of sham-operated and banded (8 and 20 weeks after TAC) of End.p53-WT and End.p53-KO mice. Size bars represent $100 \mu \mathrm{m}$. B, Quantification of CD31-positive cells per $\mathrm{mm}^{2}$. C, Quantification of the lectin-positive, i.e. perfused area. Size bars represent 100 $\mu \mathrm{m}$. The summary of the quantitative analysis in $\mathrm{n}=4-9$ mice per group. ${ }^{*} \mathrm{P}<0.05$ and $* * * \mathrm{P}<0.001$ vs. sham. Significant differences between End.p53-WT and End.p53-KO mice are indicated within the graphs. 


\subsection{Endothelial p53 Deletion Reduces Hypoxia in Hearts After TAC}

Expression of the hypoxia marker CAIX was increased in banded hearts of mice of both genotypes, but to a significantly lesser extent in End.p53-KO mice $(\mathrm{P}<0.001$; Figure 5.15A and B) compared to the End.p53-WT mice suggesting that decrease in apoptosis and increase in vascularization decreases hypoxia in hypertrophic hearts of End.p53-KO mice.

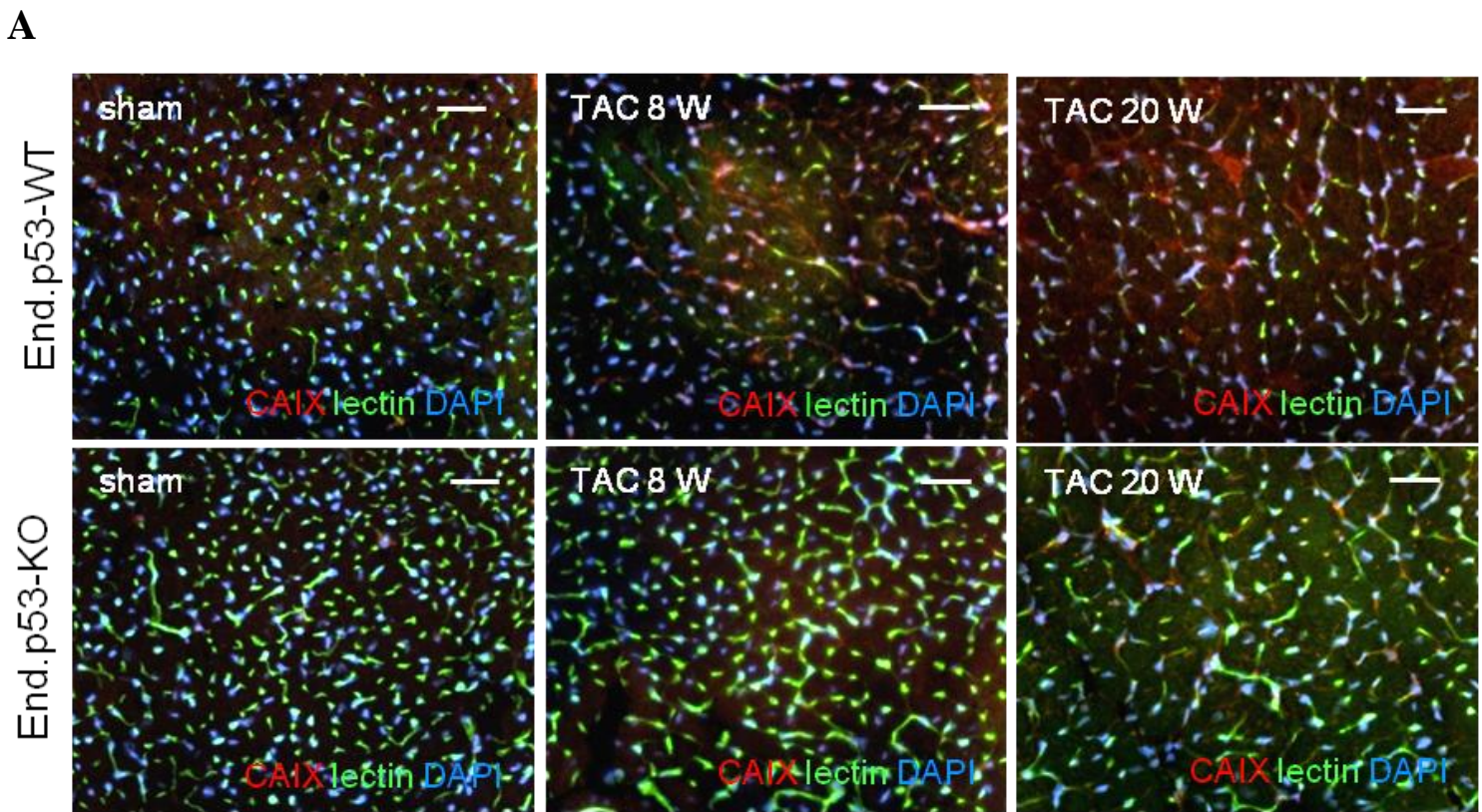

B

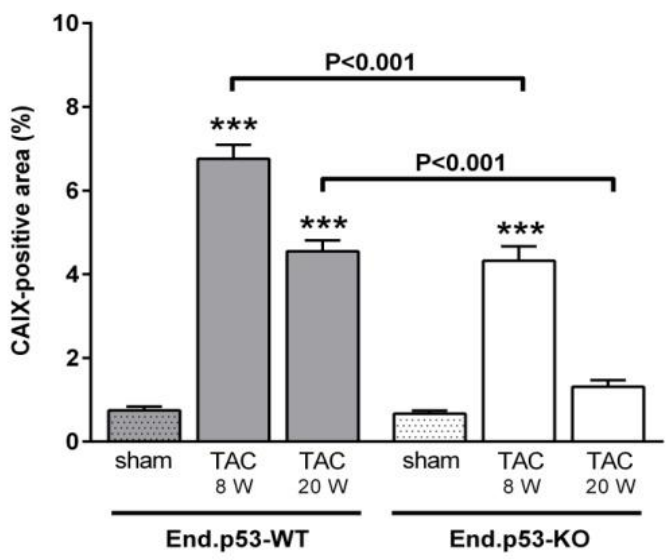

Figure 5.15. Expression of hypoxic marker in End.p53-WT and End.p53-KO mice. A, Immunostaining for CAIX to visualize hypoxia in hearts of End.p53-WT and End.p53-KO mice, sham-operated and banded (8 and 20 weeks after TAC). Size bars represent $100 \mu \mathrm{m}$. B, The summary of the quantitative analysis in $n=4-9$ mice per group. $* \mathrm{P}<0.05$ and $* * * \mathrm{P}<0.001$ vs. sham. Significant differences between End.p53-WT and End.p53-KO mice are indicated within the graphs. 


\subsection{Endothelial p53 Deletion Promotes Extra-Cardiac Angiogenesis}

To investigate the effect of endothelial p53 deletion in extra-cardiac angiogenesis, we induced unilateral hindlimb ischemia. Serial laser dopplar measurements at 14, 21 and 28 days showed an improved reperfusion (Figure 5.16.A and B) and increased capillary density (Figure 5.16. C and D) within ischemic muscles from End.p53-KO compared to End.p53-WT mice.

A

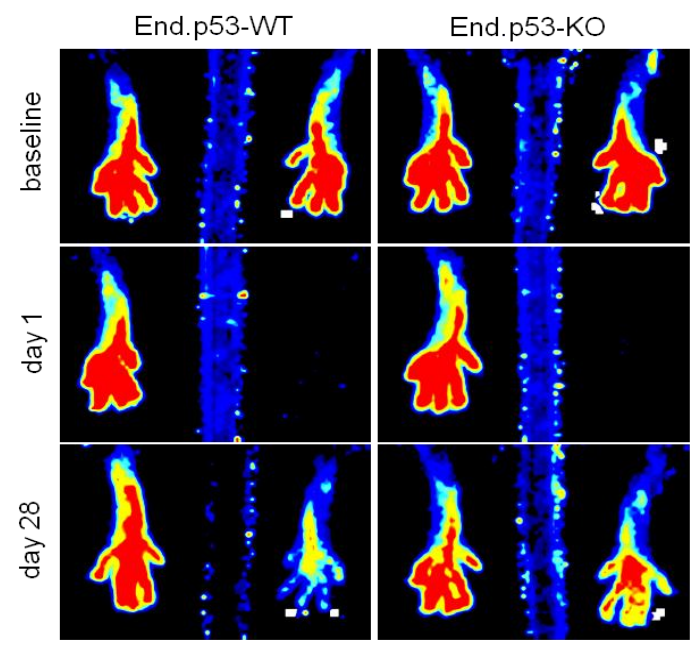

C
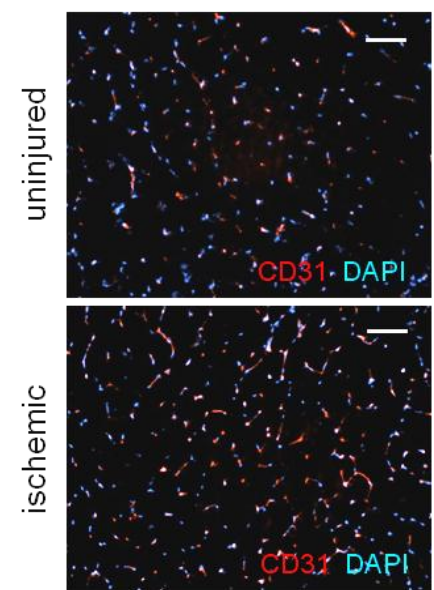

B

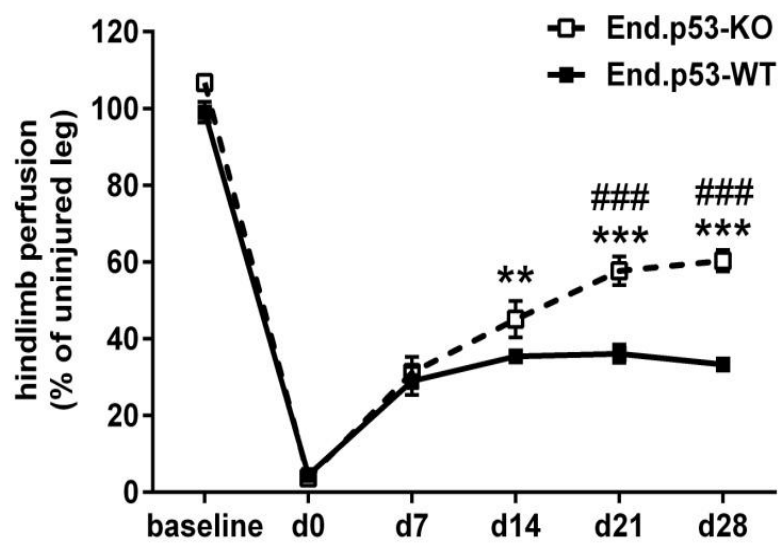

D

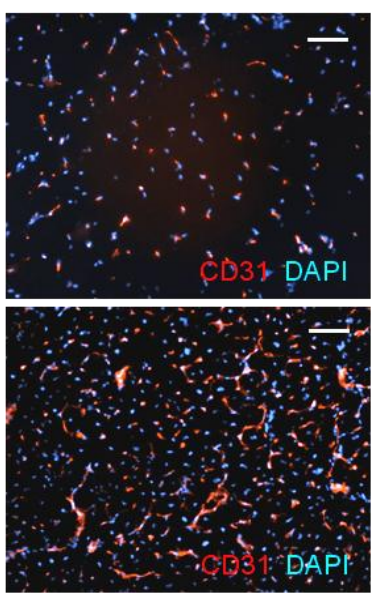

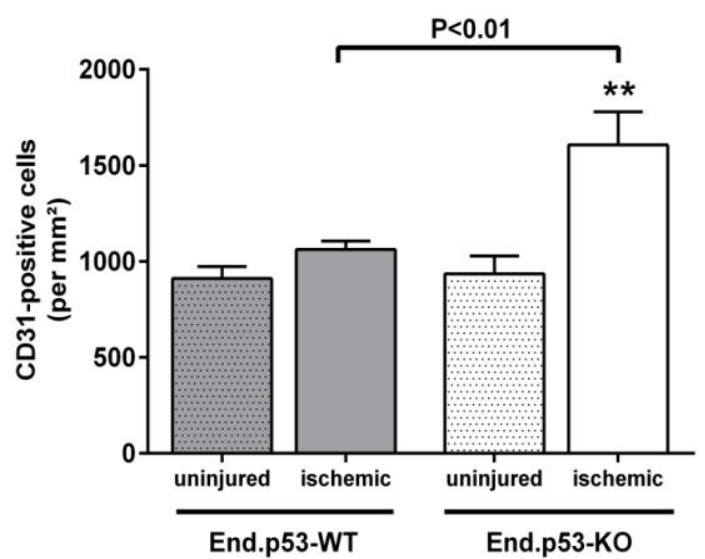

Figure 5.16. Effect of endothelial p53 deletion on neovascularization following hindlimb ischemia. A, Representative laser Doppler perfusion images before as well as on day 1 and 28 after unilateral hindlimb ischemia in End.p53-WT and End.p53-KO mice. B, Summarized findings after serial laser Doppler measurements of hindlimb perfusion in End.p53-WT $(\mathrm{n}=16)$ and End.p53-KO mice $(\mathrm{n}=14)$. **P<0.01 and 
***P<0.001 vs. baseline; \#\#\#P<0.001 vs. End.p53-WT mice. C, Representative pictures of CD31immunopositive endothelial cells in cryosections through the gastrocnemic muscle. Cell nuclei were counterstained with DAPI. Size bars represent $100 \mu \mathrm{m}$. D, Quantitative analysis of the capillary density 28 days after ischemia induction ( $\mathrm{n}=8-10$ mice per group). $* * \mathrm{P}<0.01$ vs. the contralateral, uninjured leg. Significant differences between End.p53-WT and End.p53-KO mice are indicated within the graph.

\subsection{Inactivation/ Inhibition of p53 Activity Improves Angiogenic Properties of Endothelial Cells in vitro}

To study the effect of modulation of p53 activity in angiogenic properties of endothelial cells invitro, we used nonpeptidic small-molecule nutlin-3a (stabilizing p53 by inhibiting the mdm2-p53 interaction) and pifithrin- $\alpha$ (reducing p53 activity) and DMSO as a control. Western blot showed that 24 hourrs incubation of nutlin-3a induced the expression of p53 by activating its downstream effector $\mathrm{p} 21$, which was not seen in pifithrin- $\alpha$ or DMSO treated cells (Fig 5.17. A). Immunostaining images (Fig 5.17. B) also shown that incubation of HCMEC's with nutlin-3a for $24 \mathrm{hrs}$ enhanced p53 expression.

A

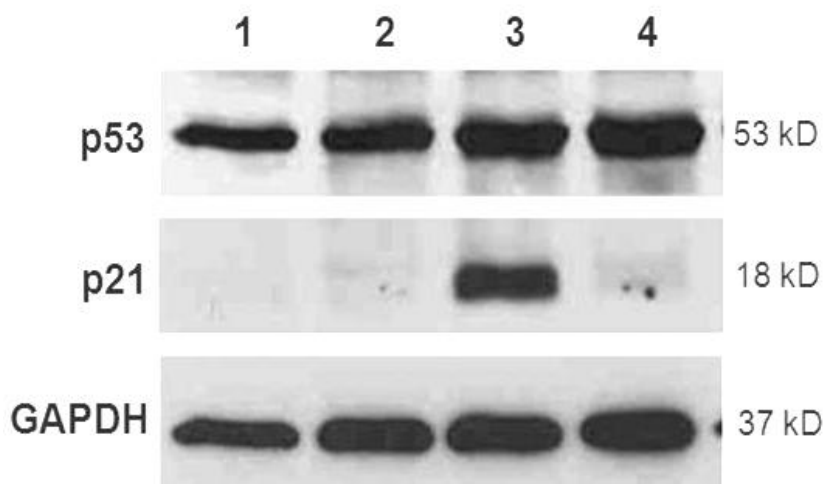

B

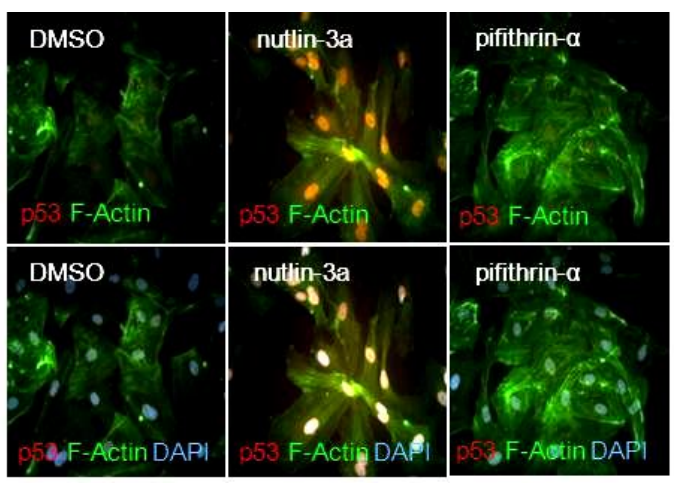

Figure 5.17. Effect of nutlin-3a and pifithrin- $\alpha$ treatment on p53 modulation of endothelial cells in vitro. A, Representative Western blot membranes showing the expression of p53 or its downstream effector p21 after incubation of human cardiac microvascular endothelial cells (HCMECs) with either PBS (1), vehicle (DMSO; 2), nutlin-3a (10 $\mu \mathrm{M} ; 3)$ or pifithrin- $\alpha(20 \mu \mathrm{M} ; 4)$. Gapdh protein was used as internal control for equal protein loading. B, Representative immunostaining pictures of HCMECs treated with either DMSO, nutlin-3a or pifithrin- $\alpha$. 
Analysis using the spheroid angiogenesis assay revealed that pifithrin- $\alpha$ treated endothelial cells were able to sprout more within 24 hours compared to nutlin-3a or DMSO treated cells supporting a role for p53 during angiogenic processes (Figure 5.18.A-C).

A

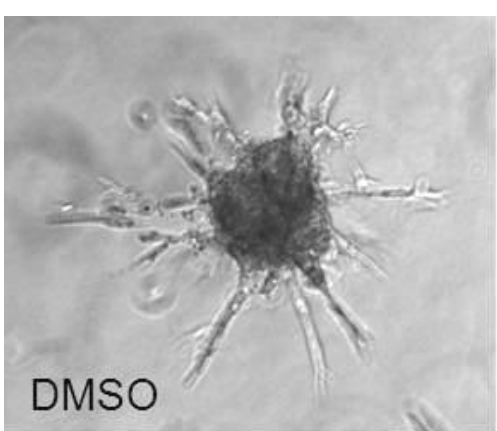

B

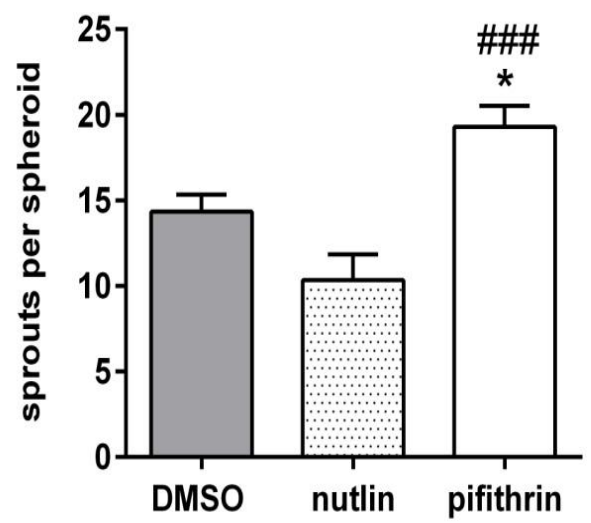

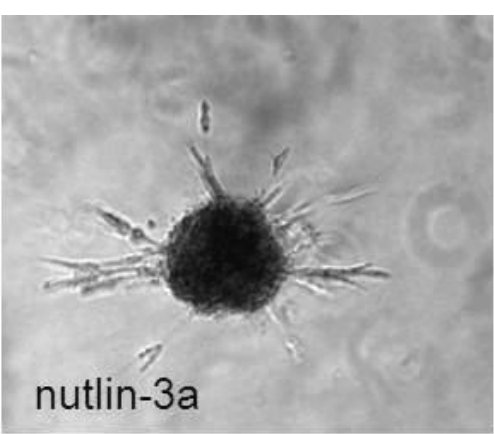

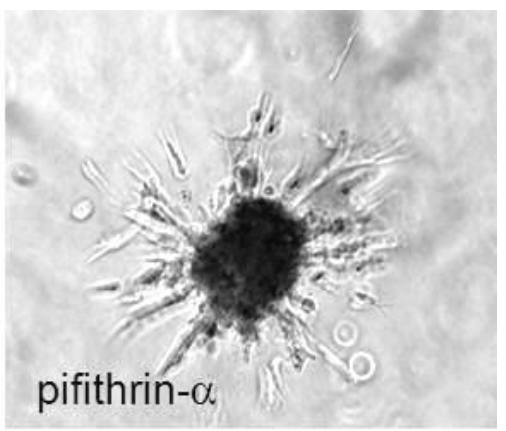

C

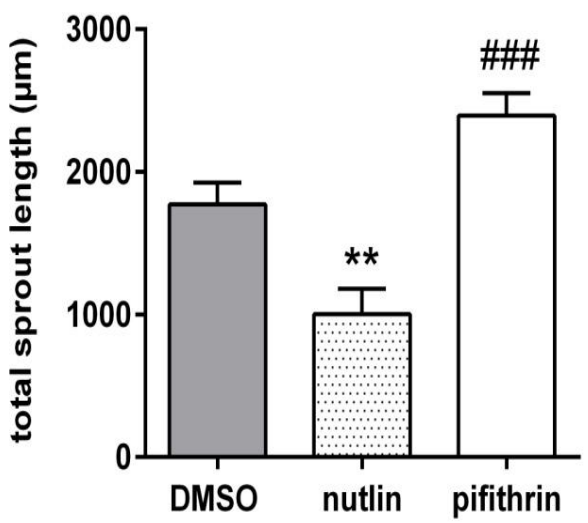

Figure 5.18. Effect of p53 modulation on the angiogenic properties of endothelial cells in vitro. A, Representative pictures of HCMEC spheroids after treatment with either DMSO, nutlin-3a or pifithrin- $\alpha$. Magnification, X200. B and C, Summary of the quantitative and statistical analysis. The number of sprouts (B) or the total sprout length $(\mathbf{C})$ per spheroid were calculated using ImagePro Plus image analysis software. $* \mathrm{P}<0.05$ and $* * \mathrm{P}<0.01$ vs. DMSO; \#\#\#P<0.001 vs. nutlin-3a, as determined using ANOVA. 


\subsection{Hearts from End.p53-KO Mice Revealed Lower Levels of Apoptosis and Improved Angiogenesis After Doxorubicin Treatment}

Doxorubicin, a cardiotoxic agent known to induce apoptosis via activation of p53, was used to treat endothelial cells resulting in higher p53 protein expression and increased levels of apoptosis (Figure 5.19. A, B). Doxorubicin was injected into End.p53-KO and WT mice as a single dose. Five days after treatment, significantly lower numbers of TUNEL-positive apoptotic cells (Figure 5.20. A-C) and higher amount of endothelial lectin-perfused blood vessels (Figure 5.20. A and D) were observed in End.p53-KO mice compared to End.p53WT mice.

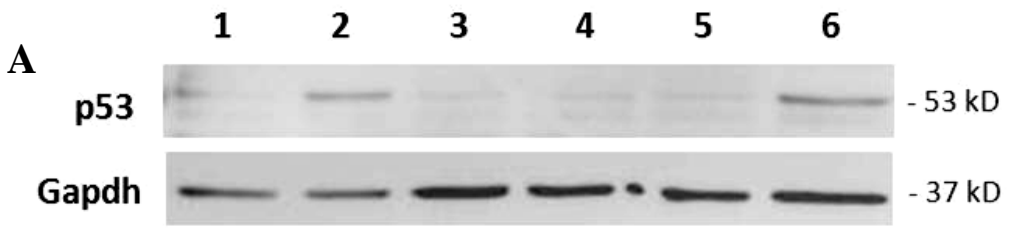

B
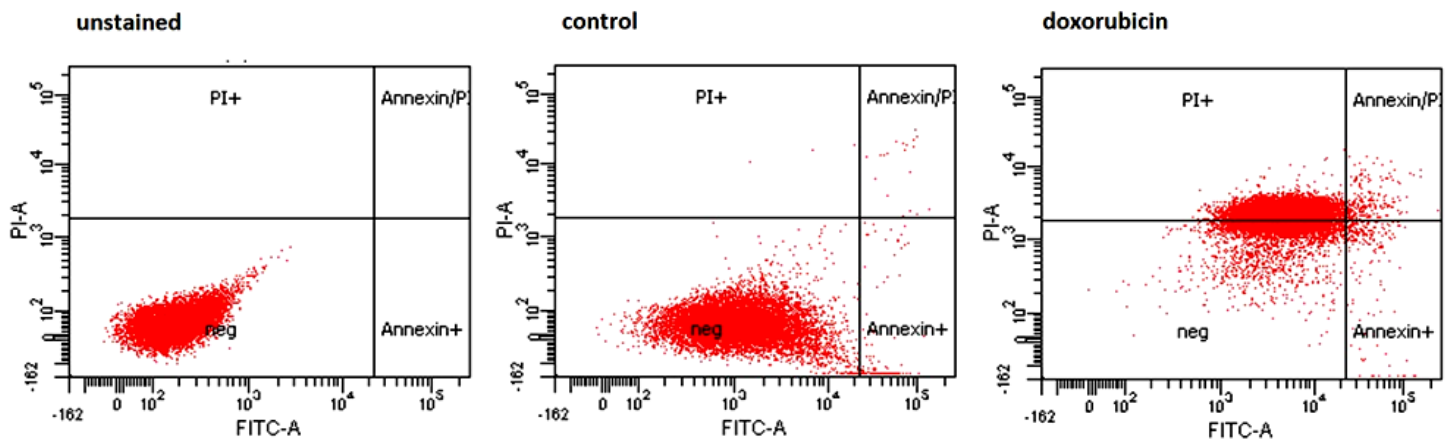

Figure 5.19. p53 expression, apoptosis and angiogenesis after doxorubicin treatment in vitro and in vivo. A, p53 protein levels were examined in HCMECs after stimulation (for 24 hours) with PBS (1) or $0.5 \mu \mathrm{M}$ doxorubicin (2) and compared to cells treated with DMSO (3) or $20 \mu \mathrm{M}$ of the p53-inhibitor pifithrin- $\alpha$ (4) as negative control, or DMSO (5) or $10 \mu \mathrm{M}$ of the p53 activator nutlin-3a (6) as positive control. B, Flow cytometry analysis of annexin $\mathrm{V}$ and propidium iodide (PI) expression revealed increased cell death in doxorubicin- compared to control-treated HCMECs (16.7 vs. 0.5\%). 
A
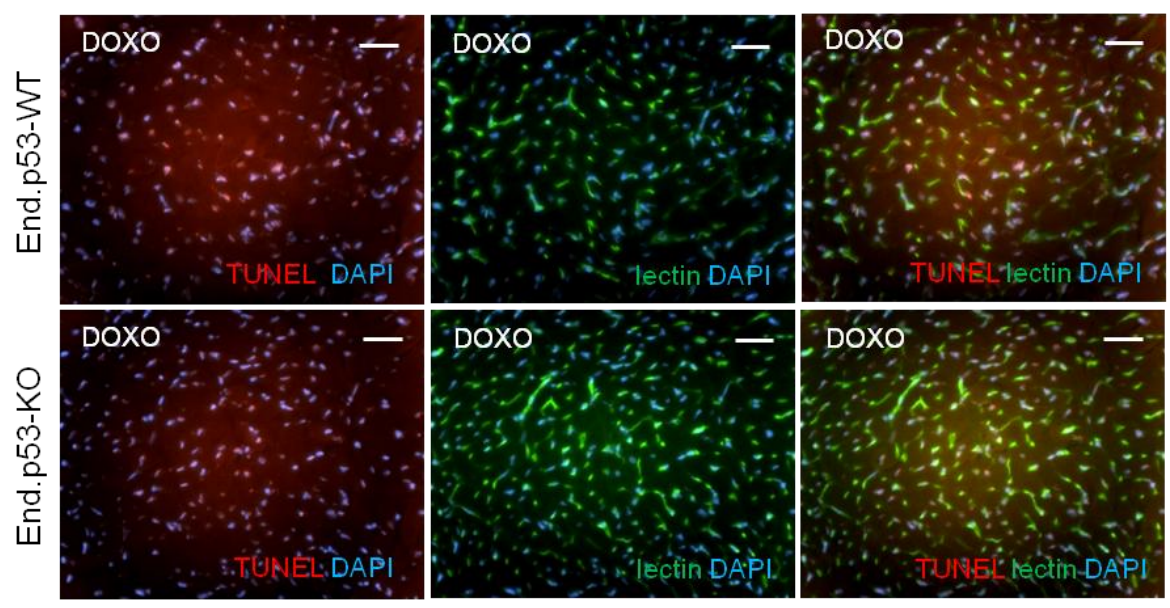

B

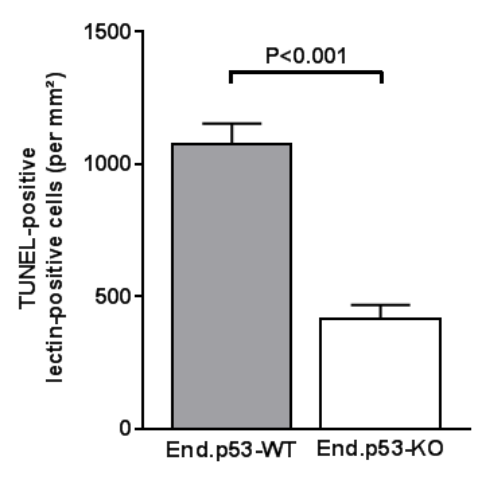

C

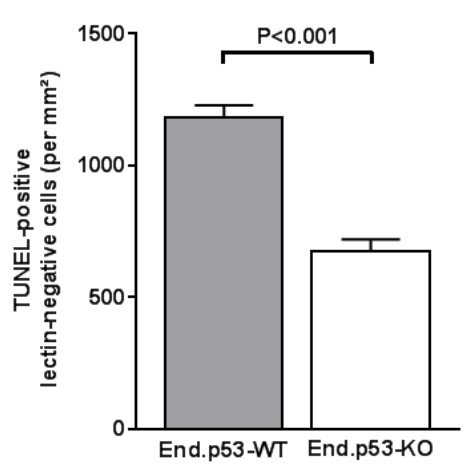

D

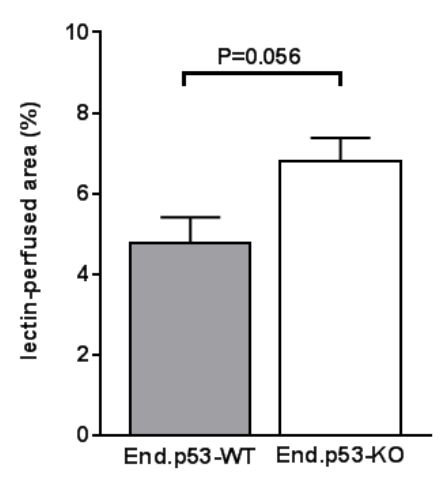

Figure 5.20. Apoptosis and angiogenesis after doxorubicin treatment in vitro and in vivo. Representative images of 2 independent experiments are shown. A-C, Histological analysis of hearts from End.p53-WT (grey bars) and End.p53-KO mice (white bars) on day 5 after a single i.p. doxorubicin (DOX) injection revealed reduced numbers of apoptotic (i.e. TUNEL-positive). D, lectin-positive and lectin-negative cells as well as increased endothelial lectin perfusion in mice with endothelial cell-specific p53 deletion.

\subsection{Endothelial p53 Deletion Attenuates Pressure Overload-Induced Cardiac Fibrosis}

The effects of endothelial p53 deletion on cardiac remodeling after TAC was examined after staining with Masson's trichrome. Fibrosis was negligible in sham-operated mice, multiple scattered foci of interstitial fibrosis were observed after TAC in End.p53-WT and to a significantly lesser extent in End.p53-KO mouse hearts, both at 8 and 20 weeks after TAC (Figure 5.21.A and B). 


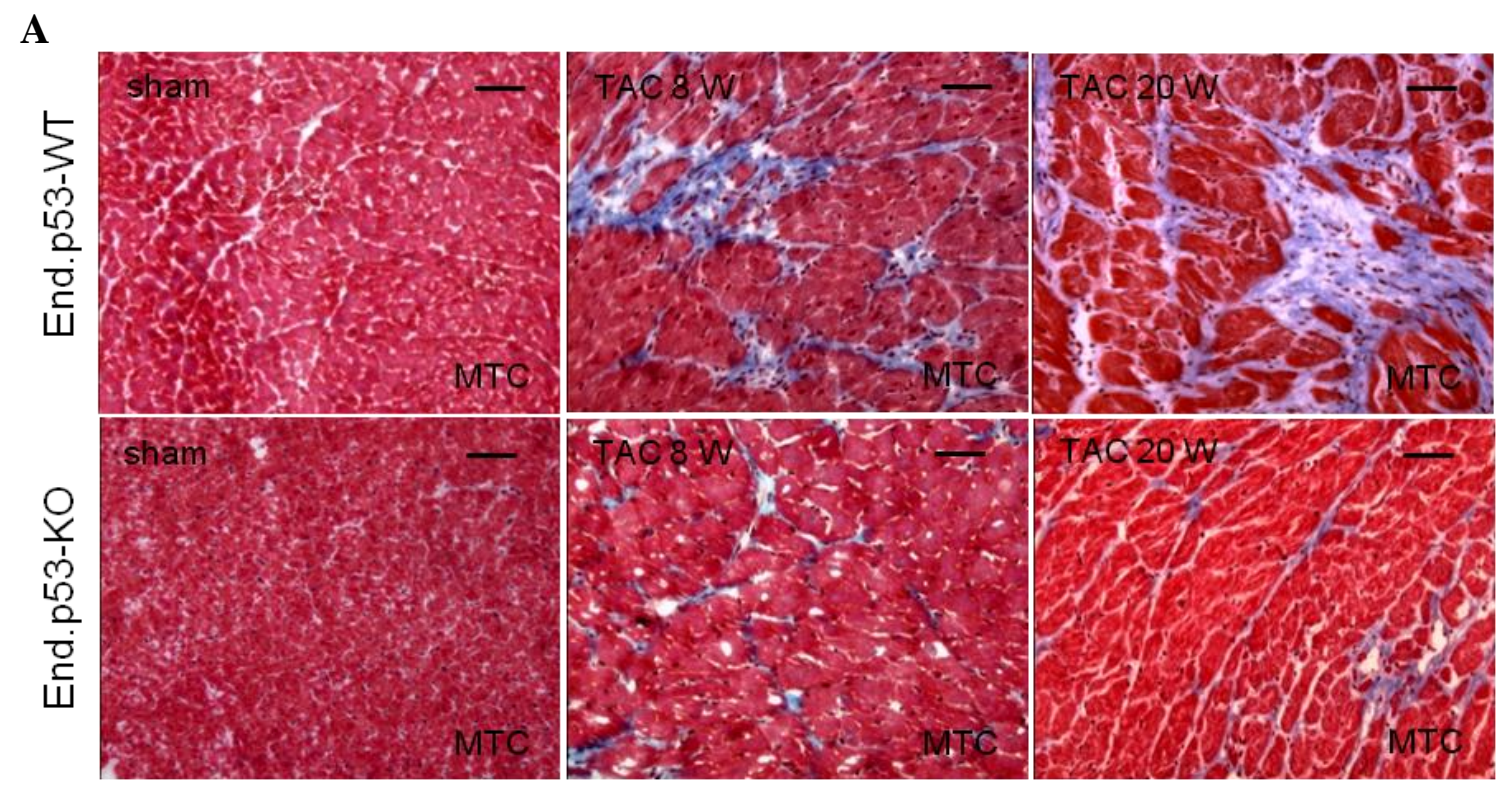

B

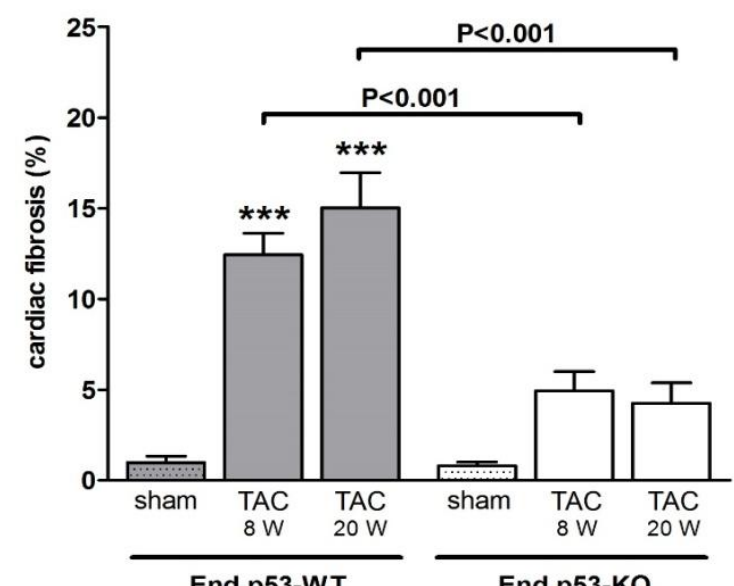

Figure 5.21. Effect of endothelial p53 deletion on cardiac fibrosis. Representative images after MTC (A) staining to visualize fibrosis in hearts of End.p53-WT and End.p53-KO mice. Size bars represent $200 \mu \mathrm{m}$. B, Summary of the quantitative analysis in $\mathrm{n}=7-15$ mice per group. $* * \mathrm{P}<0.01$ and $* * * \mathrm{P}<0.001$ vs. sham. Significant differences between End.p53-WT and End.p53-KO mice are indicated within the graphs. Significant differences between End.p53-WT and End.p53-KO mice are indicated within the graph.

Picrosirius red staining confirmed reduced amounts of interstitial collagen in hearts of TACoperated End.p53-KO compared to End.p53-WT mice (Figure 5.22. A and B). 


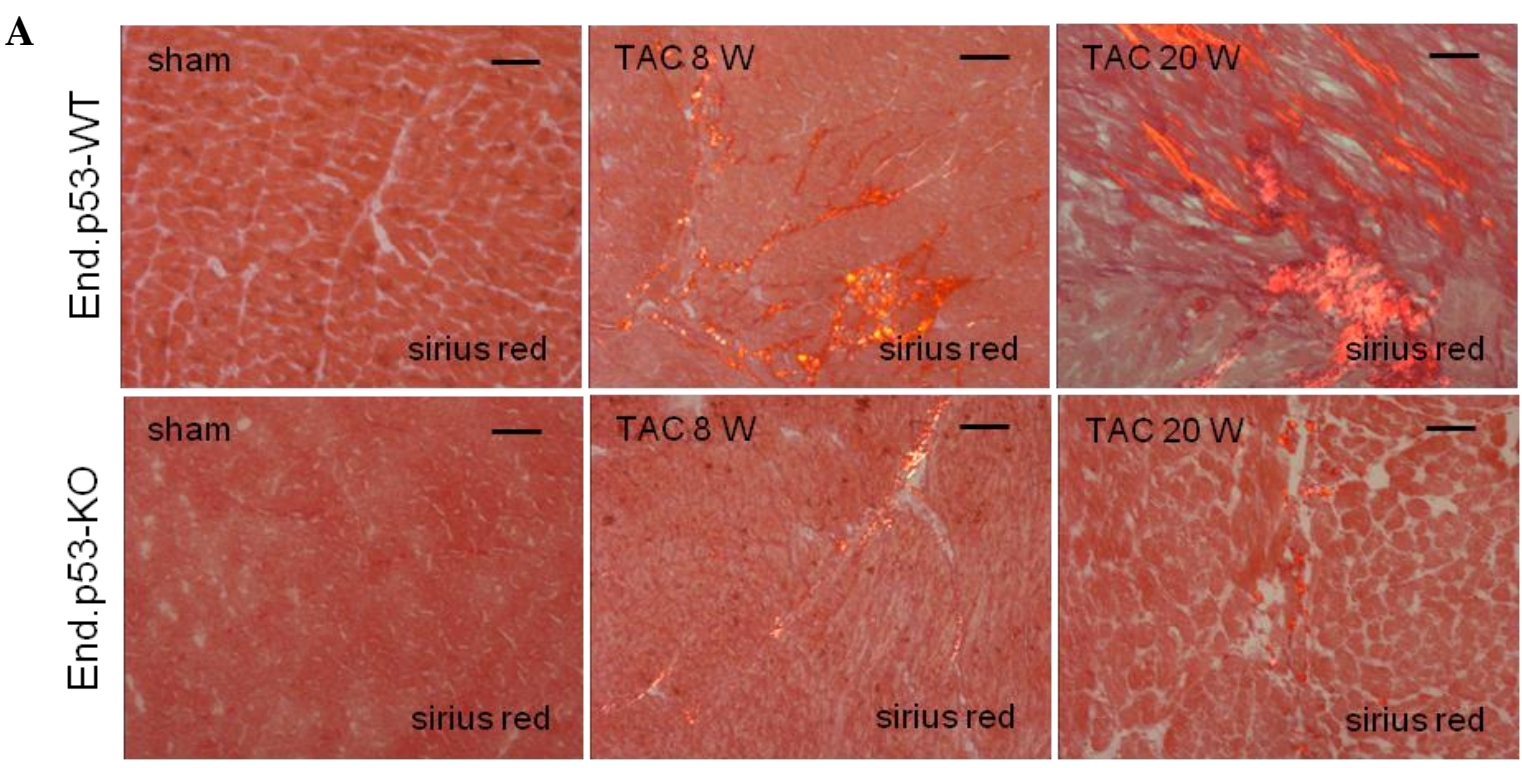

\section{B}

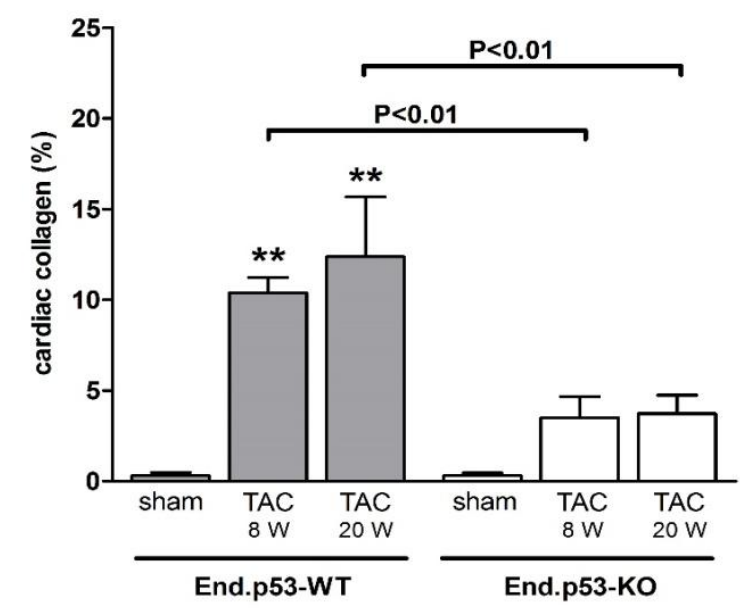

Figure 5.22. Effect of endothelial p53 deletion on accumulation of collagen after TAC. Representative images after sirius red (A) staining to visualize interstitial collagen accumulation in hearts of End.p53-WT and End.p53-KO mice. Size bars represent $200 \mu \mathrm{m}$. B, Summary of the quantitative analysis in $\mathrm{n}=7-15$ mice per group. $* * \mathrm{P}<0.01$ and $* * * \mathrm{P}<0.001$ vs. sham. Significant differences between End.p53-WT and End.p53-KO mice are indicated within the graphs.

Quantitative real time PCR analysis revealed lower cardiac mRNA levels of collagen type I alpha 1 (Col1a1) and vimentin in End.p53-KO compared to End.p53.WT mice 8 weeks after TAC (Figure 5.23.A). Moreover, increased expression of bone morphogenetic protein (Bmp)7 (Figure 5.23.B) and reduced levels of connective tissue growth factor (Ctgf) or plasminogen activator inhibitor (Pai)-1 (Figure 5.23.C) were detected in hearts of mice 
lacking endothelial p53, suggesting that both reduced production and enhanced degradation of ECM proteins may be involved in the attenuated fibrosis observed in End.p53-KO mouse hearts.

A

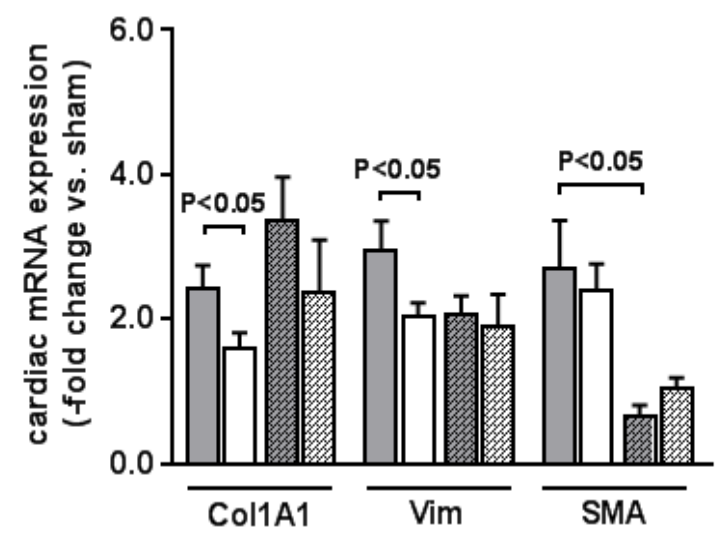

B

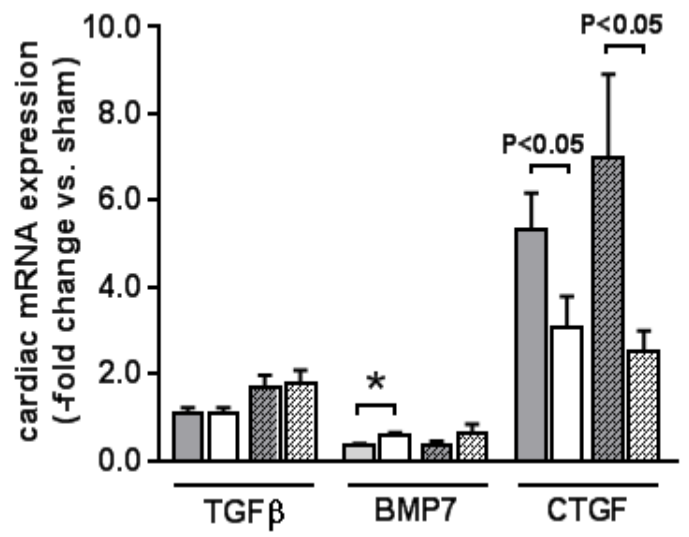

C

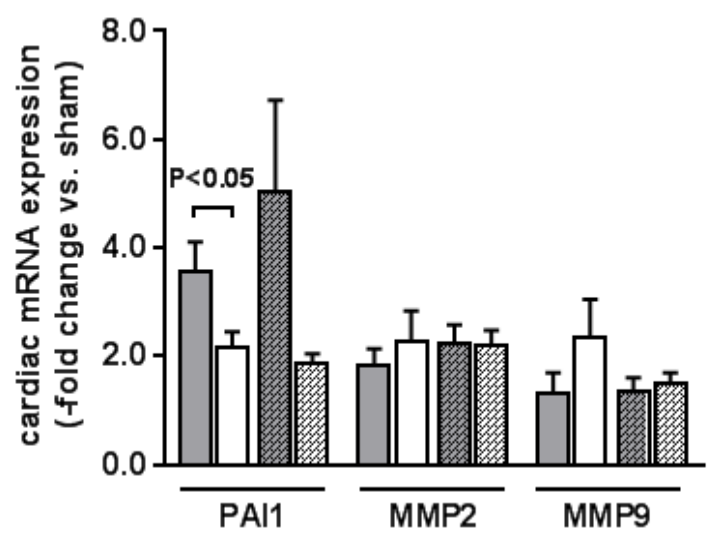

Figure 5.23. Effect of endothelial p53 deletion on cardiac fibrosis. E-G, Quantitative real time PCR analysis of the mRNA expression levels of ECM proteins and mesenchymal markers (A), or factors involved in ECM production (B) and degradation (C) in End.p53-WT and End.p53-KO mice hearts after TAC. Results were normalized to GAPDH and are expressed as -fold increase vs. sham-operated mice (set at 1; not shown). Differences between End.p53-WT and End.p53-KO mice are indicated within the graphs. 


\subsection{Deletion of p53 in Endothelial Cells Reduces Surrogate Markers of Endothelial-to-Mesenchymal Transition}

(Myo-)fibroblasts are a major source of ECM protein-producing cells in the heart and have been shown to originate, at least in part, from resident endothelial cells. ${ }^{112}$ In this regard, immunohistochemistry demonstrated that the number of cells immunopositive for both the mesenchymal marker FSP1 and the endothelial marker CD31 were reduced in hearts of End.p53-KO mice compared to their End.p53-WT counterparts (Figure 5.24.A and B).

A
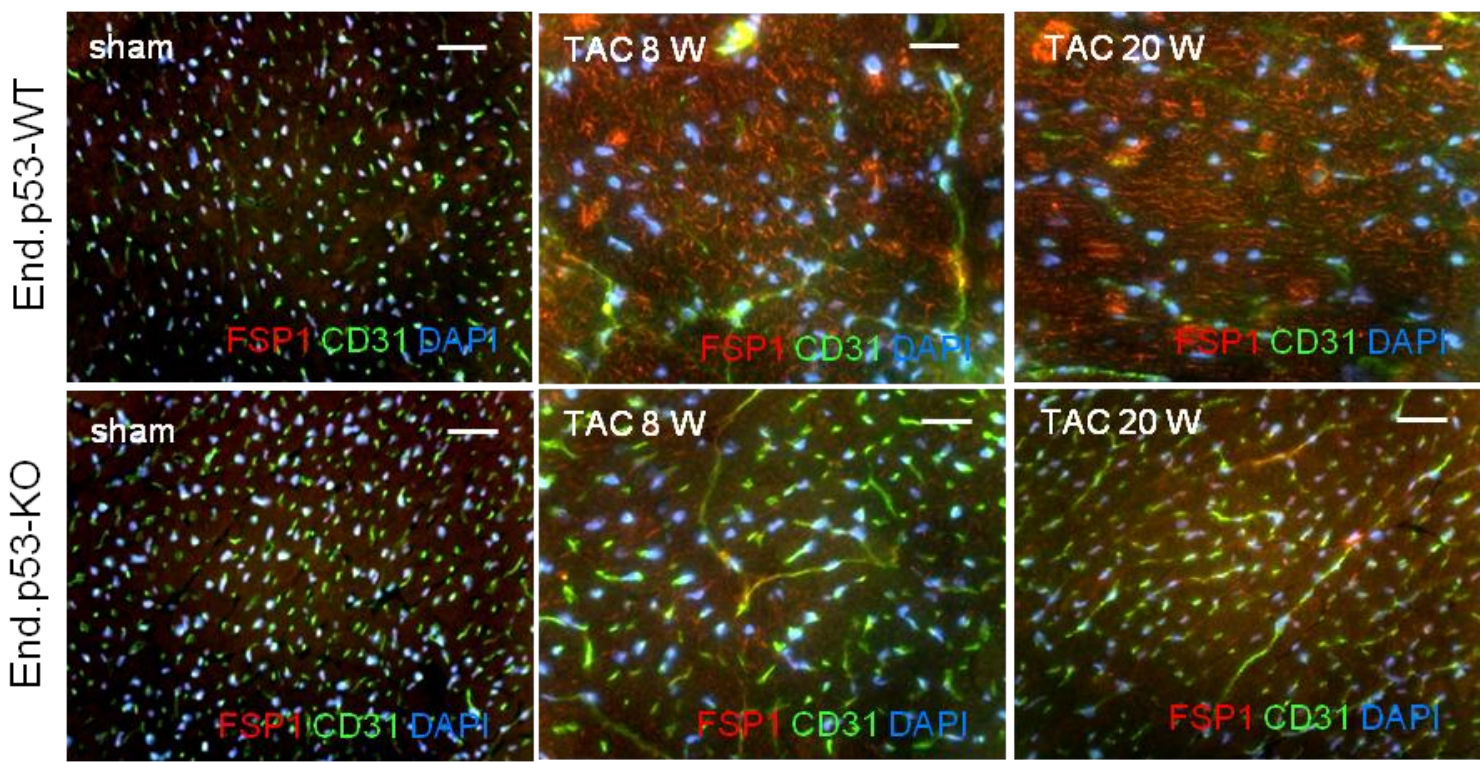

B

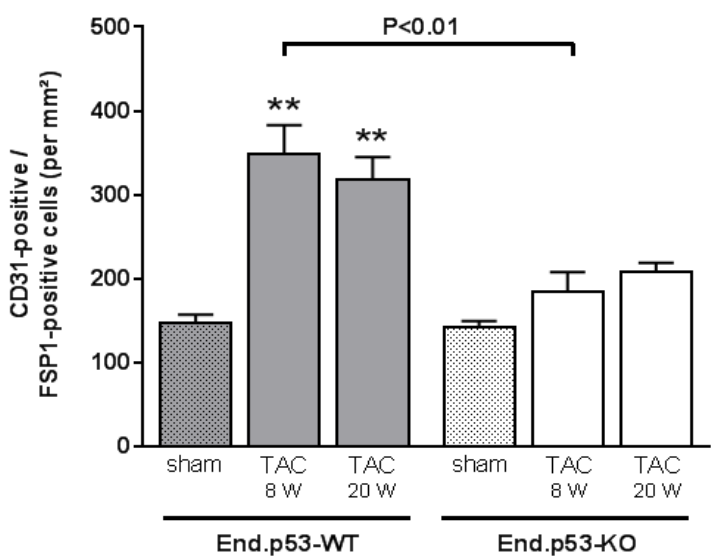

Figure 5.24. Effect of endothelial p53 deletion on markers of EndMT and myofibroblast transdifferentiation. A and B, Representative images and summarized findings after quantification of FSP$1^{+} / \mathrm{CD} 31^{+}$cells in hearts of TAC-operated End.p53-WT and End.p53-KO mice ( $\mathrm{n}=3-4$ mice per group). Size bars represent $100 \mu \mathrm{m}$. 
Similar findings were obtained after immunohistochemical detection of cells double-positive for CD31 and collagen type 1 (Figure 5.25A and B).

A

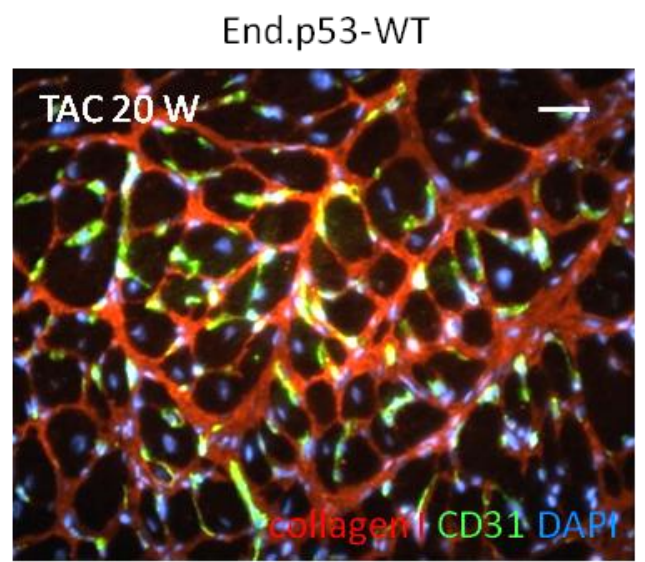

End.p53-KO

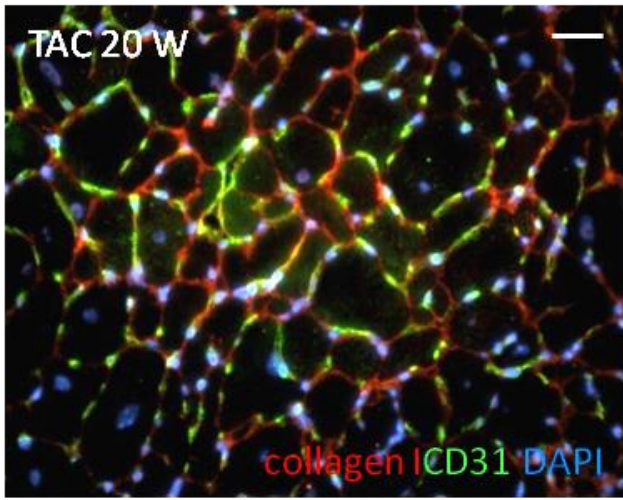

B

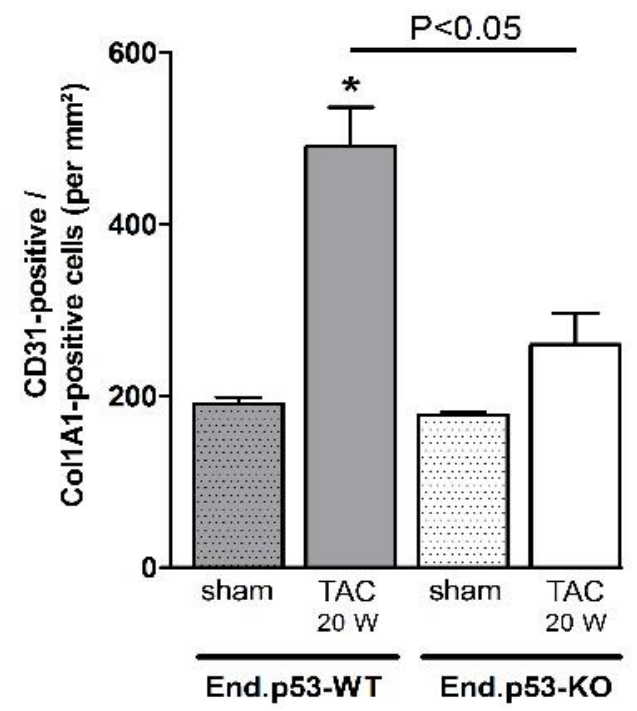

Figure 5.25. Analysis of endothelial and mesenchymal marker expression in mouse hearts after TAC. A, Representative images and $\mathbf{B}$, summarized findings after quantification of collagen type $\mathrm{I}^{+} / \mathrm{CD} 31^{+}$cells in hearts of End.p53-WT and End.p53-KO mice at 20 weeks after TAC surgery. ${ }^{*} \mathrm{P}<0.05$ vs. sham. Significant differences between End.p53-WT and End.p53-KO mice are indicated within the graph.

To test the hypothesis that reduced EndMT might have contributed to the observed differences in cardiac fibrosis, treatment of HCMECs with TGF $\beta 1$ in the presence or absence 
of nutlin-3a or pifithrin- $\alpha$ revealed that stabilization of p53 promoted EndMT surrogate marker expression, whereas inhibition of p53 reduced it (Figure 5.26.A-C).

A

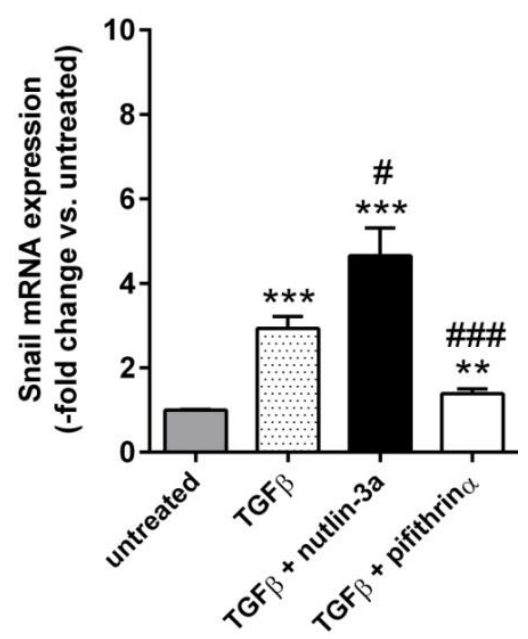

B

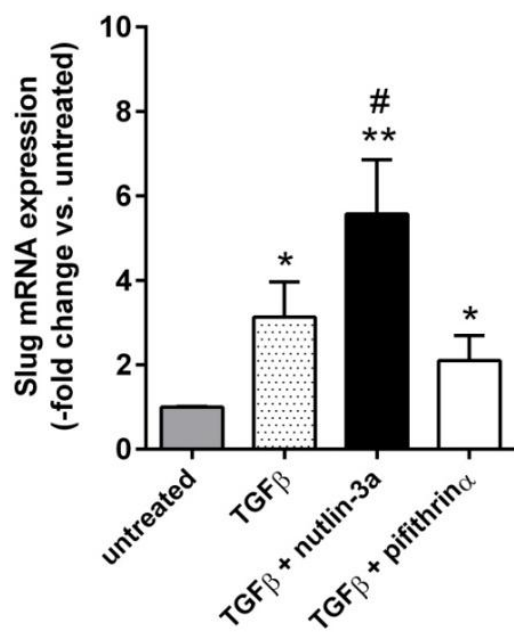

C

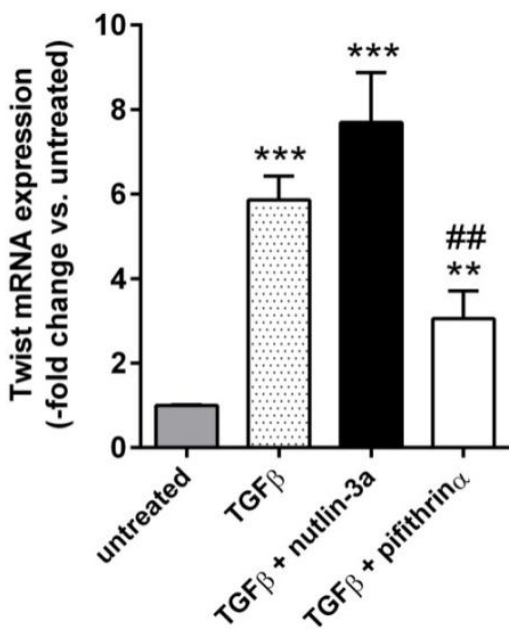

Figure 5.26. Effect of p53 activation or inhibition on TGFß-induced endothelial-to-mesenchymal transition of human cardiac microvascular endothelial cells. A-C, Quantitative real time PCR analysis of the EndMT surrogate marker Snail (A), Slug (B) and Twist (C) mRNA expression in HCMECs after treatment with TGF $\beta$, alone or in combination with nutlin-3a or pifithrin- $\alpha$ for 6 days. Similar findings were observed after

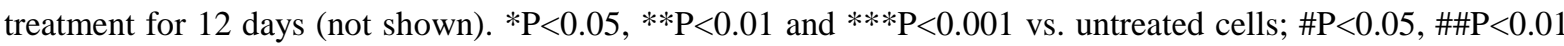
and \#\#P<0.001 vs. TGF $\beta$-treated cells.

Real time PCR analysis of whole hearts, TAC- or sham-operated (Figure 5.27.A-C) as well as of FACS-sorted CD31-immunopositive and -negative cells derived from banded hearts of either End.p53-WT or End.p53-KO mice (Figure 5.27.D-F) revealed lower mRNA expression levels of the transcription factors Snail, Slug and Twist in mice with endothelial p53 deletion. Treatment of HCMECs with TGF $\beta$ after stable transfection with scr or p53 shRNA (Figure 5.27.G-I) also shown lower expression levels of Snail, Slug and Twist transcription factors in vitro suggesting the role of p53 in EndoMT. 

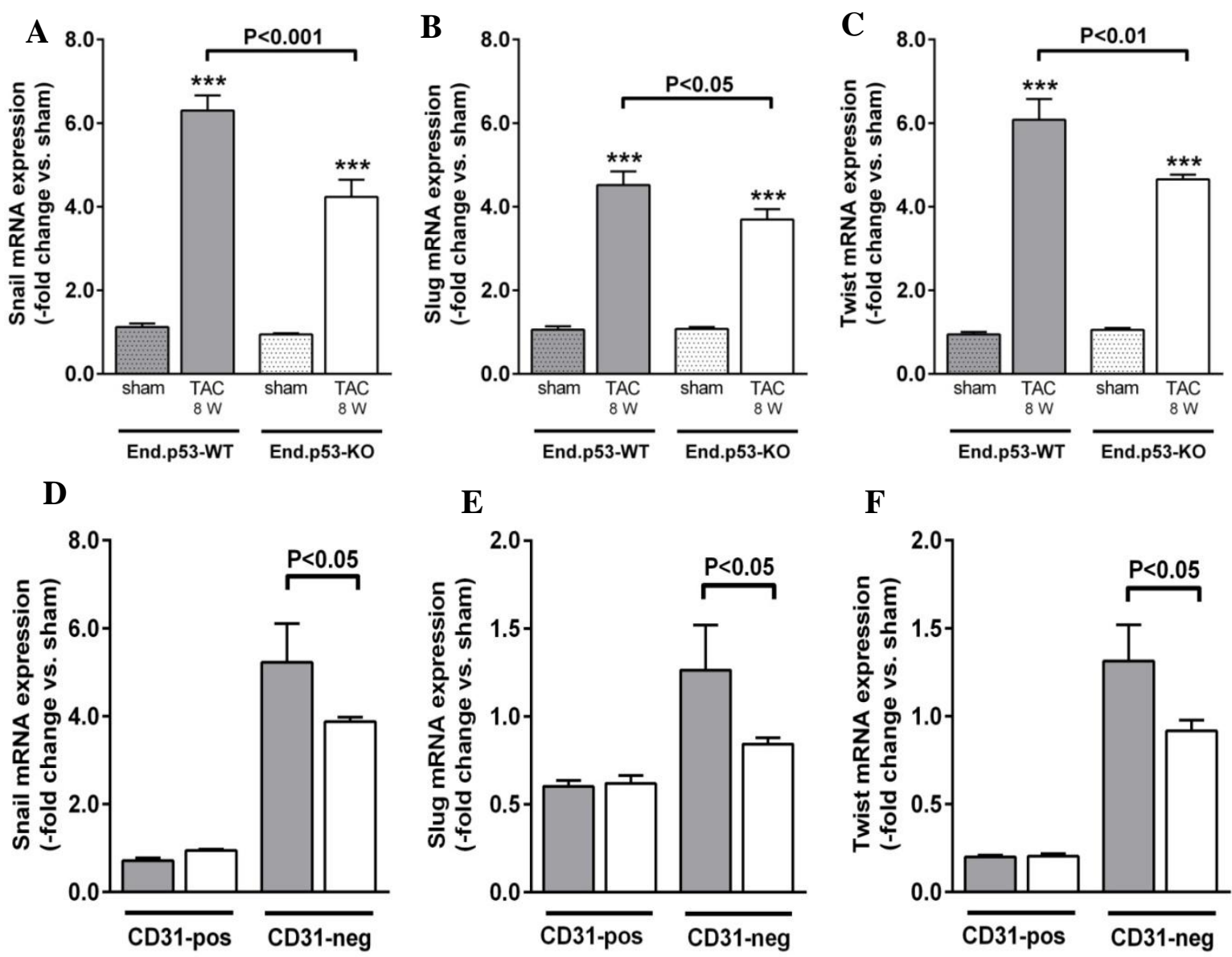

F
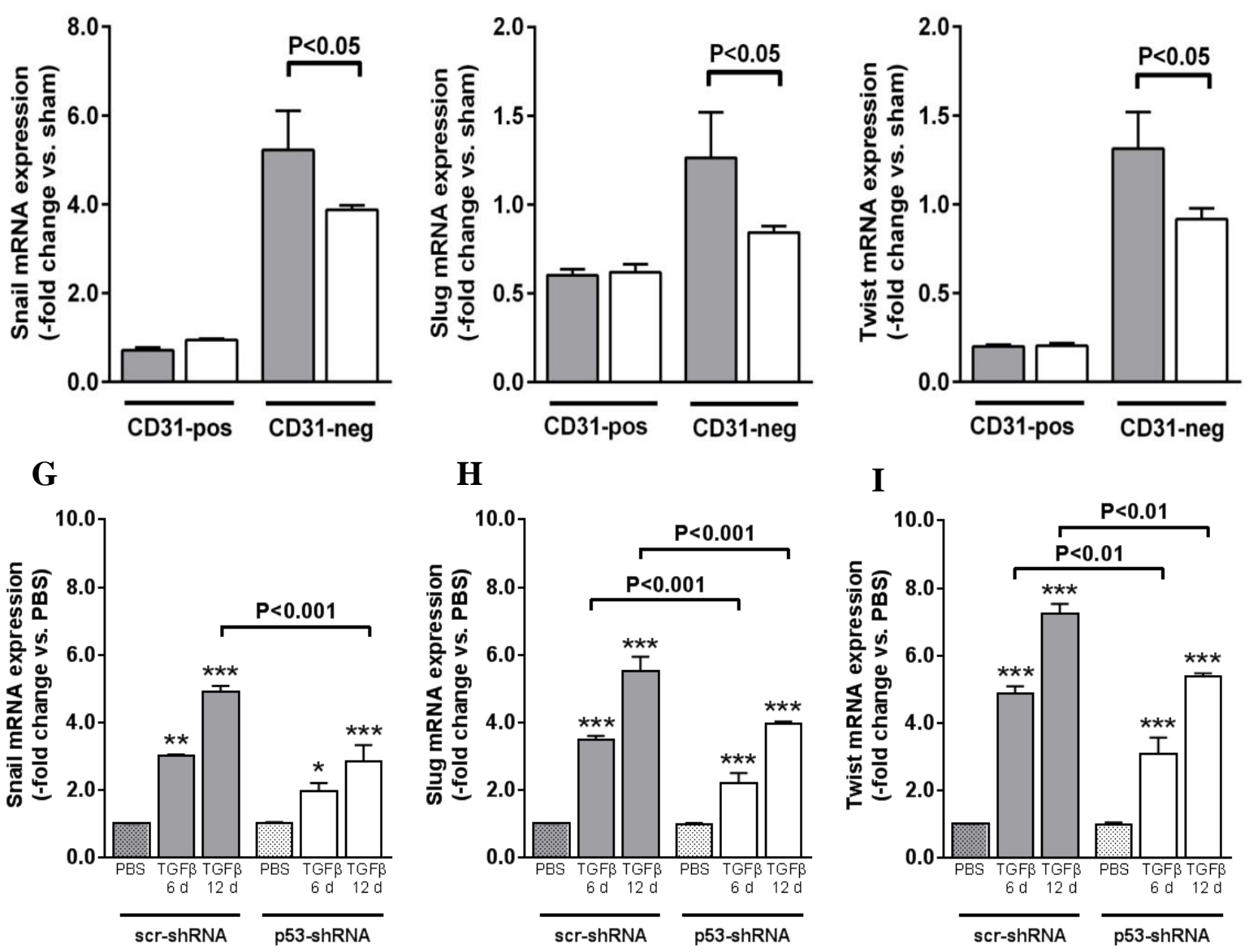

Figure 5.27. Effect of endothelial p53 deletion on markers of EndMT and myofibroblast transdifferentiation. Real time PCR analysis of whole mouse hearts (A-C; $\mathrm{n}=9$ per group) or FACS-sorted CD31-positive or -negative cells (D-F; $n=3$ per group) from End.p53-KO (white bars) and End.p53-WT mice (grey bars) 8 weeks after TAC for Snail (A and $\mathbf{D})$, Slug (B and $\mathbf{E})$ and Twist $(\mathbf{C}$ and $\mathbf{F})$ mRNA. ***P<0.001 vs. sham. Significant differences between End.p53-WT and End.p53-KO mice are indicated within the graphs. G-I, HCMECs were stable transfected with lentiviral p53-shRNA or negative control (scr) shRNA vector, treated with PBS or TGF $\beta 1(10 \mathrm{ng} / \mathrm{mL})$ for 6 or 12 days and the expression of EndMT markers examined by real time PCR analysis. ${ }^{*} \mathrm{P}<0.05, * * \mathrm{P}<0.01$ and $* * * \mathrm{P}<0.001$ vs. PBS-treated cells. Significant differences between $\mathrm{p} 53$ shRNA and scr shRNA transfected cells are indicated within the graphs. 


\section{Discussion}

\subsection{Major Findings of the Study}

In this study, we examined the importance and causal role of endothelial p53 expression for cardiac remodeling and the development of heart failure induced by chronic pressure overload in mice.

Our main findings are that inducible deletion of p53 in endothelial cells of adult mice ameliorated pressure overload-induced cardiac fibrosis and attenuated the progressive LV dilation and systolic pump dysfunction present in End.WT-p53 mice, resulting in improved survival. Mechanistically, we provide experimental evidence that endothelial p53 deletion is associated with reduced numbers of apoptotic endothelial, but also non-endothelial cells (presumably cardiomyocytes, fibroblasts or smooth muscle cells) and increased cardiac Hif $1 \alpha$ and Vegf levels, and prevents the rarefaction of capillary endothelial cells observed with developing heart failure thus improving cardiac perfusion and oxygenation. In addition to regulating genes involved in ECM production and turnover (in particular, Bmp7, Ctgf and Pai-1), p53 expression may also play a role in the phenotypic switching of endothelial cells into matrix-producing fibroblast-like cells. Thus, our data support the importance of endothelial cells during pressure overload cardiac remodeling and also suggest a direct link between endothelial cell p53 expression and cardiac fibrosis.

\subsection{Role of p53 in Myocardial Apoptosis and Heart Failure}

The tumor suppressor protein p53 is activated in response to a variety of cellular stress signals. DNA damage, but also oxidative stress, hypoxia or cytokine stimulation may induce apoptotic cell death via p53 and transcriptional activation of genes involved in cell cycle control and growth arrest. ${ }^{112 ; 138} \mathrm{~A}$ role for $\mathrm{p} 53$ in the pathogenesis of heart disease is supported by findings of increased apoptotic cell numbers and p53 protein levels in the myocardium of patients with advanced heart disease, ${ }^{81}$ correlating with the transition to heart failure. ${ }^{80}$ Activation of $\mathrm{p} 53$ and associated genes was also reported in experimental rat, ${ }^{82}$ sheep ${ }^{139}$ or $\operatorname{dog}^{140}$ models of heart failure. Of note, quantitative analysis of TUNEL-positive cells in banded mouse hearts revealed $0.7 \%$ apoptotic cardiomyocytes and $1 \%$ apoptotic non- 
cardiomyocytes, half of which were identified as CD31-positive endothelial cells. ${ }^{141}$ Activation of p53 may be causally involved in the development of heart failure. For example, mice with global p53 deficiency are characterized by increased cardiac angiogenesis and protection from acute myocardial infarction- or TAC-induced heart failure. ${ }^{87}$ Similar findings were observed in mice systemically treated with the synthetic p53 inhibitor pifithrin- $\alpha{ }^{85}$ Overexpression of CHIP, an endogenous p53 inhibitor, prevented myocardial apoptosis and ameliorated ventricular remodeling after myocardial infarction. ${ }^{142}$ Global p53 deficiency ${ }^{143}$ as well as cardiomyocyte-specific expression of dominant-negative $\mathrm{p} 53^{144}$ also protected against the cardiotoxic effects of doxorubicin, whereas a recent study in mice with cardiomyocytespecific p53 deletion did not observe any cardioprotection. ${ }^{145}$ In addition to the possibility that doxorubicin-induced apoptosis occurs in a p53-independent manner, the latter findings also suggest that p53 expression in other cell types (e.g. endothelial cells) may be more important, a hypothesis that is supported by the findings of the present study. Previous studies have shown that serum of heart failure patients induces endothelial cell apoptosis ${ }^{146}$ and that circulating levels of apoptotic microparticles provide independent prognostic information in patients with advanced heart failure. ${ }^{147}$ However, the cardioprotective effects of endothelial cell-specific p53 deletion have not been directly addressed so far.

To investigate the cardioprotective effects of endothelial cell-specific p53 deletion in mice, we generated mice with inducible endothelial cell-specific p53 deletion (End.p53-KO mice). We hypothesised that stabilization of endothelial cells by preventing apoptosis of endothelial cells may improve vascularisation and can lead to better heart function during hypertrophic conditions. To investigate our hypothesis, cardiac hypertrophy was induced in End.p53-KO and End.p53-WT female mice by TAC surgery. We analyzed both groups at 8 and 20 weeks time point for cardiac hypertrophy and function. As we hypothesized, End.p53-KO mice expressed significantly lower levels of p53 and p 21 mRNA and protein after TAC at 8 and 20 weeks time points compared to wildtype mice (Figure 5.6 and 5.7). Although it is known that p53 knockout mice develop tumors with a high incidence, ${ }^{148}$ our endothelial-specific p53 deletion mice did not show any signs of tumorigenesis, at least macroscopically and until 24 months of their birth. 


\subsection{Deletion of p53 in Endothelial Cells Exhibit Less Apoptosis and Hypoxia}

Endothelial cell apoptosis is a well-known anti-angiogenic mechanism. It occurs during vascular pruning, i.e. the removal of aberrant neovessels, and represents a vital step during secondary vascular network formation. Angiogenesis inhibitors have been shown to promote apoptosis, ${ }^{149}$ whereas angiogenic growth factors protect endothelial cells from programmed cell death. ${ }^{150}$ Overexpression of p53 was shown to inhibit endothelial differentiation and angiogenesis. ${ }^{151}$ Possible mechanisms by which p53 may limit angiogenesis include the transcriptional activation of angiogenesis inhibitors, such as collagen prolyl hydroxylase ${ }^{152}$ or increased production of Mmp2 or Mmp9 resulting in the release of anti-angiogenic factors, such as collagen-derived arresten. ${ }^{153}$ Interestingly, heat shock transcription factor-1 was found to protect hearts after TAC by suppressing p53 and upregulating Hif $1 \alpha$ in endothelial cells. ${ }^{154}$ Recent paper has shown that during hypoxic condition $\mathrm{p} 53$ has contrasting roles in the regulation of Vegf expression. During the initial stages of acute hypoxia, p53 cooperate with Hif- $1 \alpha$ to up regulate Vegf expression. On the other hand, after extended exposure to hypoxia or to genotoxic stress, p53 inhibit Vegf expression in a p21/retinoblastoma (Rb)dependent manner. ${ }^{155}$ In our study, we found that, End.p53-KO mice significantly reduced the apoptosis of endothelial and non endothelial cells (Figure.5.9 and 5.10) after TAC compared to the wildtype mice, suggesting that deletion of p53 in endothelial cells stabilise the endothelial cells by reducing the apoptosis and thereby accompanied the growing myocytes.

Doxorubicin (Dox) is a member of Anthracycline (ANT) family of anticancer drugs. These drugs, when given to patients, may induce cardiotoxicity leading to cardiomyocyte apoptosis, myocardial fibrosis, and congestive heart failure. p53 plays a important role in Dox-induced apoptosis in cardiomyocytes and endothelial cells. Recently, it was reported that deletion of p53 in cardiomyocytes is not sufficient to block myocardial fibrosis and cardiomyocyte apoptosis. ${ }^{145}$ In our study, we showed that endothelial specific deletion of p53 significantly reduced apoptosis in endothelial and non endothelial cells and increased the capillary density (Figure 5.20. A-D) suggesting again that endothelial cell stabilization or survival plays a vital role in maintaining the normal cardiac function. 


\subsection{Role of Hypoxia in Cardiac Hypertrophy and Angiogenesis}

Cardiac hypertrophy leads to myocardial ischemia, which is caused by the lack of perfusion and insufficient supply of nutrients. Lack of perfusion or blood supply leads to hypoxia and reduction in oxygen levels. Hypoxia can lead to cell death in chronic conditions. Hypoxiainducing factors (HIFs) like Hif1 $\alpha$ are stabilized under hypoxic conditions and induce adaptive angiogenesis by transcriptionally activating pro-angiogenic genes, such as Vegf. At the later stages of hypertrophy, p53 accumulates and interacts with Hif $1 \alpha$ to disrupt the adaptive angiogenic activity, promoting its degradation and also by activating pro-apoptotic molecules like Bax, PUMA and Bcl2. ${ }^{87: 156}$ p53 and Hif1 $\alpha$ both are involved in cell adaptation to various stress conditions, they are known to be involved in similar processes such as apoptosis, cell cycle control or metabolism, as shown in Figure 6.1. ${ }^{157}$

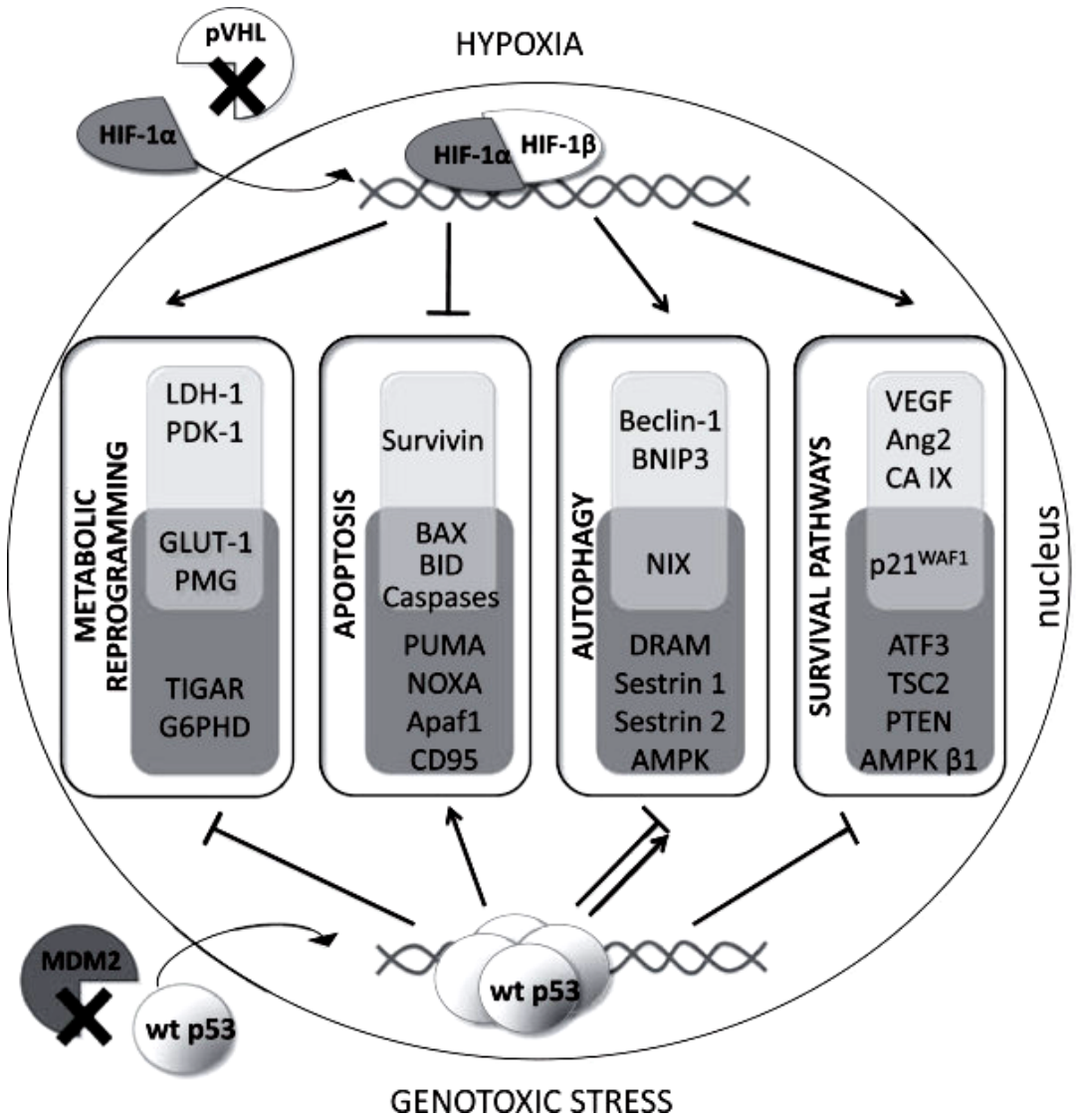

(Figure adapted from Obacz J et al Molecular Cancer 2013;12:93)

Figure 6.1. HIF-1 and/or p53 regulated genes mediating adaptation to cellular stresses through activation of different pathways. 
In our study, we found that endothelial cell-specific p53 deletion is associated with lower expression of surrogate markers of hypoxia (i.e. CAIX) and elevated Hifl $\alpha$ and Vegf levels in response to pressure overload in vivo or hypoxia in vitro. We found that Hif $1 \alpha$ and Vegf are induced significantly higher in End.p53-KO mice during the initial stages of hypertrophy (at 7 day time point) compared to the wildtype mice after TAC (Figure 5.12). Our in vitro findings in p53 KO-HCMECs and normal HCMECs also confirmed our in vivo data by showing that when exposed to hypoxic state mimicked by $\mathrm{CoCl}_{2}$, deletion of p53 in endothelial cells significantly enhanced Hif1 $\alpha$ protein levels (Figure 5.13) in response to (chemical) hypoxia compared to normal wildtype endothelial cells.

\subsection{Deletion of p53 Specifically in Endothelial Cells Enhances Cardiac and Extra-Cardiac Angiogenesis and Postponed Heart Failure after TAC}

Several studies have shown that the progression of cardiac hypertrophy towards heart failure is associated with rarefication of the cardiac microvasculature, which is then unable to support the increased oxygen and nutrient demands of the hypertrophied myocardium. ${ }^{21-24}$ Building on these previous findings, we could now show that deletion of p53 in endothelial cells prevents the reduction in cardiac capillary density after TAC resulting in enhanced perfusion of the hypertrophied heart and elevated Hif1 $\alpha$ and Vegf levels. The anti-angiogenic effects of p53 could be confirmed in vitro as well as after induction of unilateral hindlimb ischemia in vivo. As we discussed earlier that deletion of p53 specifically in endothelial cells have significantly increased the Hifl $\alpha$ and Vegf levels after TAC, we looked at the angiogenesis in the heart tissue after 8 and 20 weeks after TAC. Angiogenesis was significantly increased in End.p53-KO mice at both the time points compared to wild type (Figure 5.14). Extracardiac angiogenesis or neovascularization was also significantly increased in End.p53-KO mice after unilateral hindlimb ischemia (Figure 5.16).

Importantly, our findings also suggest that prevention of endothelial cell death and stabilization of cardiac capillaries may delay the progressive LV dilation and the decline of systolic pump function (Figure 5.4). In contrast to previous studies showing that augmentation of angiogenesis promotes myocardial hypertrophy even in the absence of an initiating stimulus, ${ }^{25}$ endothelial p53 deletion reduced the extent of cardiac hypertrophy at later time points (i.e. 20 weeks after TAC), as determined by wall thickness, heart weight or 
cardiomyocyte area (Figure 5.5). Future studies will have to determine the pathways underlying the cross-talk between endothelial cells and cardiomyocytes during hypertrophy and how they are affected by p53. On the other hand, cardiac deletion of PUMA (p53upregulated modulator of apoptosis) was found to postpone heart failure after TAC and to improve cardiac remodeling without affecting hypertrophy or angiogenesis. ${ }^{158}$

\subsection{Deletion of $\mathbf{p 5 3}$ Specifically in Endothelial Cells Attenuates TAC Induced Cardiac Fibrosis and Endothelial-Mesenchymal Transition (EndoMT)}

Hearts of End.p53-KO mice exhibited markedly reduced fibrosis (Figure 5.21) and expressed lower amounts of collagen type I (Figure 5.22, 5.25) and other mesenchymal markers (Figure 5.24), which may have contributed to the preservation of cardiac pump function. Possible mechanisms underlying the reduced cardiac fibrosis in mice lacking p53 in endothelial cells may include indirect effects, such as improved cardiac perfusion limiting hypoxia and the death of adjacent cardiomyocytes. In this regard, reduced numbers of p53 (Figure 5.8), activated caspase-3 (Figure 5.9) and TUNEL-positive endothelial lectin-positive as well as endothelial lectin-negative cells (Figure 5.10) were observed in hearts of End.p53-KO mice.

In addition, several genes involved in ECM synthesis and degradation are known to be directly regulated by p53 and may have acted in a paracrine manner on adjacent cardiomyocytes and/or fibroblasts. ${ }^{91}$ For example (Figure 5.23), End.p53-KO mouse hearts expressed significantly lower amounts of Ctgf, a major regulator of tissue fibrosis, and elevated Ctgf expression and increased liver fibrosis was recently reported in mice with hepatocyte-specific p53 activation. ${ }^{159}$ Moreover, the p53-regulated factors Pai-1 and Mmp9 are not only involved in the proteolytic release of angiogenesis inhibitors, but also modulate ECM degradation. Of note, constitutive deletion of p53 in endothelial cells was recently reported to be associated with a worse outcome and more severe cardiac fibrosis in mice after total body irradiation, ${ }^{145}$ although differences in the type of injury may have contributed to this discrepancy.

Reduced numbers of Fsp $1^{+} / \mathrm{CD} 31^{+}$-double positive cells (Figure 5.24) or mRNA expression of surrogate markers of EndMT (i.e. Snail, Slug and Twist in Figure 5.27) in hearts of 
End.p53-KO mice after TAC suggest that differences in EndMT may also have contributed to the observed protection against fibrosis mice lacking endothelial p53. Previous studies have shown that TGF $\beta$-induced fibroblast activation and EndMT are significant contributors to myocardial fibrosis. ${ }^{112}$ Although cardiac levels of TGF $\beta$, a major inducer of EndMT, did not differ between both genotypes, elevated levels of BMP7, previously shown to preserve the endothelial phenotype and to counteract TGF $\beta$-induced organ fibrosis, ${ }^{160}$ were detected in hearts of End.p53-KO mice and might have contributed to our observations. Future studies will have to examine in more detail the exact role of p53 in EndMT regulation.

Our results also suggest that modulating endothelial p53 expression may represent an interesting therapeutic target. In this regard, atorvastatin was found to restore ischemic limb loss in diabetes by activation of the $\mathrm{Akt} / \mathrm{mdm} 2$ pathway and augmentation of p53 degradation, ${ }^{161}$ and modulation of endothelial p53 may also underlie the beneficial effects of statins on markers of endothelial function and LV remodeling in heart failure patients. ${ }^{162}$ Our observations may be especially relevant in elderly subjects, a population at increased risk for heart failure, as previous studies could show that ageing is associated with elevated levels of the p53. For example, prolonged passaging (mimicking senescence) of human vein endothelial cells was associated with p53 accumulation, ${ }^{163}$ whereas mice with a truncated p53 mutation resulting in p53 activation were protected against tumor formation, but exhibited an early-onset phenotype consistent with accelerated ageing. ${ }^{164}$ 


\section{Conclusions}

Inducible, endothelial cell-specific deletion of p53 in adult mice

- prevents endothelial and non-endothelial cell apoptosis,

- induces Hif-1 $\alpha$ and Vegf expression,

- enhances cardiac and extra-cardiac new vessel formation,

- reduces cardiac fibrosis and EndMT,

- improves heart function and survival after TAC.

In conclusion, our findings suggest that accumulation of p53 in endothelial cells contributes to the increased endothelial cell apoptosis and the rarefication of cardiac endothelial cells during cardiac hypertrophy and promotes the development of cardiac fibrosis and LV dysfunction through, at least in part, impaired cardiac perfusion and altered ECM remodeling. Attenuation of endothelial cell loss and apoptotic death and preservation of the cardiac microvasculature during pathologic hypertrophy may thus represent a promising approach to improve pressure overload-induced cardiac remodeling and to prevent the transition to heart failure. Moreover, our data also support the hypothesis of other investigators that apoptosis plays a critical role in the pathogenesis of heart failure. ${ }^{165-167}$ 


\section{References}

1. Dorn GW, Robbins J, Sugden PH. Phenotyping hypertrophy: eschew obfuscation. Circ Res. 2003;92:1171-1175.

2. Heineke J, Molkentin JD. Regulation of cardiac hypertrophy by intracellular signalling pathways. Nat Rev Mol Cell Biol. 2006;7:589-600.

3. Hunter JJ, Chien KR. Signaling pathways for cardiac hypertrophy and failure. N Engl J Med. 1999;341:1276-1283.

4. Tirziu D, Chorianopoulos E, Moodie KL, Palac RT, Zhuang ZW, Tjwa M, Roncal C, Eriksson U, Fu Q, Elfenbein A, Hall AE, Carmeliet P, Moons L, Simons M. Myocardial hypertrophy in the absence of external stimuli is induced by angiogenesis in mice. J Clin Invest. 2007;117:3188-3197.

5. Frey N, Olson EN. Cardiac hypertrophy: the good, the bad, and the ugly. Annu Rev Physiol. 2003;65:45-79.

6. MEERSON FZ. On the mechanism of compensatory hyperfunction and insufficiency of the heart. Cor Vasa. 1961;3:161-177.

7. Chien KR, Knowlton KU, Zhu H, Chien S. Regulation of cardiac gene expression during myocardial growth and hypertrophy: molecular studies of an adaptive physiologic response. FASEB J. 1991;5:3037-3046.

8. Iemitsu M, Miyauchi T, Maeda S, Sakai S, Kobayashi T, Fujii N, Miyazaki H, Matsuda M, Yamaguchi I. Physiological and pathological cardiac hypertrophy induce different molecular phenotypes in the rat. Am J Physiol Regul Integr Comp Physiol. 2001;281:R2029-R2036.

9. Medugorac I. Collagen content in different areas of normal and hypertrophied rat myocardium. Cardiovasc Res. 1980;14:551-554.

10. Pelliccia A, Maron BJ, De Luca R, Di Paolo FM, Spataro A, Culasso F. Remodeling of left ventricular hypertrophy in elite athletes after long-term deconditioning. Circulation. 2002;105:944-949.

11. Andersen JB, Rourke BC, Caiozzo VJ, Bennett AF, Hicks JW. Physiology: postprandial cardiac hypertrophy in pythons. Nature. 2005;434:37-38.

12. Dorn GW. The fuzzy logic of physiological cardiac hypertrophy. Hypertension. 2007;49:962-970.

13. Schannwell CM, Zimmermann T, Schneppenheim M, Plehn G, Marx R, Strauer BE. Left ventricular hypertrophy and diastolic dysfunction in healthy pregnant women. Cardiology. 2002;97:73-78.

14. Hunter S, Robson SC. Adaptation of the maternal heart in pregnancy. Br Heart J. 1992;68:540-543.

15. Ro A, Frishman WH. Peripartum cardiomyopathy. Cardiol Rev. 2006;14:35-42. 
16. Litten RZ, III, Martin BJ, Low RB, Alpert NR. Altered myosin isozyme patterns from pressure-overloaded and thyrotoxic hypertrophied rabbit hearts. Circ Res. 1982;50:856-864.

17. Arai M, Matsui H, Periasamy M. Sarcoplasmic reticulum gene expression in cardiac hypertrophy and heart failure. Circ Res. 1994;74:555-564.

18. Kong SW, Bodyak N, Yue P, Liu Z, Brown J, Izumo S, Kang PM. Genetic expression profiles during physiological and pathological cardiac hypertrophy and heart failure in rats. Physiol Genomics. 2005;21:34-42.

19. Bernardo BC, Weeks KL, Pretorius L, McMullen JR. Molecular distinction between physiological and pathological cardiac hypertrophy: experimental findings and therapeutic strategies. Pharmacol Ther. 2010;128:191-227.

20. Mirotsou M, Dzau VJ, Pratt RE, Weinberg EO. Physiological genomics of cardiac disease: quantitative relationships between gene expression and left ventricular hypertrophy. Physiol Genomics. 2006;27:86-94.

21. De Boer RA, Pinto YM, Van Veldhuisen DJ. The imbalance between oxygen demand and supply as a potential mechanism in the pathophysiology of heart failure: the role of microvascular growth and abnormalities. Microcirculation. 2003;10:113-126.

22. Hein S, Arnon E, Kostin S, Schonburg M, Elsasser A, Polyakova V, Bauer EP, Klovekorn WP, Schaper J. Progression from compensated hypertrophy to failure in the pressure-overloaded human heart: structural deterioration and compensatory mechanisms. Circulation. 2003;107:984-991.

23. Hudlicka O, Brown M, Egginton S. Angiogenesis in skeletal and cardiac muscle. Physiol Rev. 1992;72:369-417.

24. Shiojima I, Sato K, Izumiya Y, Schiekofer S, Ito M, Liao R, Colucci WS, Walsh K. Disruption of coordinated cardiac hypertrophy and angiogenesis contributes to the transition to heart failure. J Clin Invest. 2005;115:2108-2118.

25. Tirziu D, Chorianopoulos E, Moodie KL, Palac RT, Zhuang ZW, Tjwa M, Roncal C, Eriksson U, Fu Q, Elfenbein A, Hall AE, Carmeliet P, Moons L, Simons M. Myocardial hypertrophy in the absence of external stimuli is induced by angiogenesis in mice. J Clin Invest. 2007;117:3188-3197.

26. Tao Z, Chen B, Tan X, Zhao Y, Wang L, Zhu T, Cao K, Yang Z, Kan YW, Su H. Coexpression of VEGF and angiopoietin-1 promotes angiogenesis and cardiomyocyte proliferation reduces apoptosis in porcine myocardial infarction (MI) heart. Proc Natl Acad Sci U S A. 2011;108:2064-2069.

27. Olofsson B, Pajusola K, Kaipainen A, von Euler G, Joukov V, Saksela O, Orpana A, Pettersson RF, Alitalo K, Eriksson U. Vascular endothelial growth factor B, a novel growth factor for endothelial cells. Proc Natl Acad Sci U S A. 1996;93:2576-2581.

28. Nor JE, Christensen J, Mooney DJ, Polverini PJ. Vascular endothelial growth factor (VEGF)-mediated angiogenesis is associated with enhanced endothelial cell survival and induction of Bcl-2 expression. Am J Pathol. 1999;154:375-384. 
29. Friehs I, Margossian RE, Moran AM, Cao-Danh H, Moses MA, del Nido PJ. Vascular endothelial growth factor delays onset of failure in pressure-overload hypertrophy through matrix metalloproteinase activation and angiogenesis. Basic Res Cardiol. 2006;101:204-213.

30. Yancopoulos GD, Davis S, Gale NW, Rudge JS, Wiegand SJ, Holash J. Vascularspecific growth factors and blood vessel formation. Nature. 2000;407:242-248.

31. Risau W, Flamme I. Vasculogenesis. Annu Rev Cell Dev Biol. 1995;11:73-91.

32. Cox CM, Poole TJ. Angioblast differentiation is influenced by the local environment: FGF-2 induces angioblasts and patterns vessel formation in the quail embryo. Dev Dyn. 2000;218:371-382.

33. Drake CJ. Embryonic and adult vasculogenesis. Birth Defects Res $C$ Embryo Today. 2003;69:73-82.

34. Carmeliet P. Mechanisms of angiogenesis and arteriogenesis. Nat Med. 2000;6:389395.

35. D'Amore PA, Thompson RW. Mechanisms of angiogenesis. Annu Rev Physiol. 1987;49:453-464.

36. Munoz-Chapuli R, Quesada AR, Angel MM. Angiogenesis and signal transduction in endothelial cells. Cell Mol Life Sci. 2004;61:2224-2243.

37. Risau W, Sariola H, Zerwes HG, Sasse J, Ekblom P, Kemler R, Doetschman T. Vasculogenesis and angiogenesis in embryonic-stem-cell-derived embryoid bodies. Development. 1988;102:471-478.

38. Folkman J. Angiogenesis in cancer, vascular, rheumatoid and other disease. Nat Med. 1995;1:27-31.

39. Nor JE, Polverini PJ. Role of endothelial cell survival and death signals in angiogenesis. Angiogenesis. 1999;3:101-116.

40. Hobson B, Denekamp J. Endothelial proliferation in tumours and normal tissues: continuous labelling studies. Br J Cancer. 1984;49:405-413.

41. Dai J, Rabie AB. VEGF: an essential mediator of both angiogenesis and endochondral ossification. J Dent Res. 2007;86:937-950.

42. Folkman J, Merler E, Abernathy C, Williams G. Isolation of a tumor factor responsible for angiogenesis. J Exp Med. 1971;133:275-288.

43. Leung DW, Cachianes G, Kuang WJ, Goeddel DV, Ferrara N. Vascular endothelial growth factor is a secreted angiogenic mitogen. Science. 1989;246:1306-1309.

44. Senger DR, Galli SJ, Dvorak AM, Perruzzi CA, Harvey VS, Dvorak HF. Tumor cells secrete a vascular permeability factor that promotes accumulation of ascites fluid. Science. 1983;219:983-985. 
45. Rahimi N. The ubiquitin-proteasome system meets angiogenesis. Mol Cancer Ther. 2012;11:538-548.

46. Maglione D, Guerriero V, Viglietto G, Delli-Bovi P, Persico MG. Isolation of a human placenta cDNA coding for a protein related to the vascular permeability factor. Proc Natl Acad Sci U S A. 1991;88:9267-9271.

47. Joukov V, Pajusola K, Kaipainen A, Chilov D, Lahtinen I, Kukk E, Saksela O, Kalkkinen N, Alitalo K. A novel vascular endothelial growth factor, VEGF-C, is a ligand for the Flt4 (VEGFR-3) and KDR (VEGFR-2) receptor tyrosine kinases. EMBO J. 1996;15:1751.

48. Michiels C. Endothelial cell functions. J Cell Physiol. 2003;196:430-443.

49. Semenza GL. Hypoxia-inducible factors: mediators of cancer progression and targets for cancer therapy. Trends Pharmacol Sci. 2012;33:207-214.

50. Rissanen TT, Vajanto I, Hiltunen MO, Rutanen J, Kettunen MI, Niemi M, Leppanen P, Turunen MP, Markkanen JE, Arve K, Alhava E, Kauppinen RA, Yla-Herttuala S. Expression of vascular endothelial growth factor and vascular endothelial growth factor receptor-2 (KDR/Flk-1) in ischemic skeletal muscle and its regeneration. Am J Pathol. 2002;160:1393-1403.

51. Tuomisto TT, Rissanen TT, Vajanto I, Korkeela A, Rutanen J, Yla-Herttuala S. HIFVEGF-VEGFR-2, TNF-alpha and IGF pathways are upregulated in critical human skeletal muscle ischemia as studied with DNA array. Atherosclerosis. 2004;174:111120.

52. Nyberg P, Xie L, Kalluri R. Endogenous inhibitors of angiogenesis. Cancer Res. 2005;65:3967-3979.

53. Green DR. Apoptotic pathways: the roads to ruin. Cell. 1998;94:695-698.

54. Gerber HP, McMurtrey A, Kowalski J, Yan M, Keyt BA, Dixit V, Ferrara N. Vascular endothelial growth factor regulates endothelial cell survival through the phosphatidylinositol 3'-kinase/Akt signal transduction pathway. Requirement for Flk1/KDR activation. J Biol Chem. 1998;273:30336-30343.

55. Gerber HP, Dixit V, Ferrara N. Vascular endothelial growth factor induces expression of the antiapoptotic proteins Bcl-2 and A1 in vascular endothelial cells. J Biol Chem. 1998;273:13313-13316.

56. Araki S, Simada Y, Kaji K, Hayashi H. Role of protein kinase C in the inhibition by fibroblast growth factor of apoptosis in serum-depleted endothelial cells. Biochem Biophys Res Commun. 1990;172:1081-1085.

57. Stromblad S, Cheresh DA. Integrins, angiogenesis and vascular cell survival. Chem Biol. 1996;3:881-885.

58. Stromblad S, Becker JC, Yebra M, Brooks PC, Cheresh DA. Suppression of p53 activity and p21WAF1/CIP1 expression by vascular cell integrin alphaVbeta3 during angiogenesis. J Clin Invest. 1996;98:426-433. 
59. Brooks PC, Montgomery AM, Rosenfeld M, Reisfeld RA, Hu T, Klier G, Cheresh DA. Integrin alpha $\mathrm{v}$ beta 3 antagonists promote tumor regression by inducing apoptosis of angiogenic blood vessels. Cell. 1994;79:1157-1164.

60. Brooks PC, Clark RA, Cheresh DA. Requirement of vascular integrin alpha v beta 3 for angiogenesis. Science. 1994;264:569-571.

61. Lopez-Farre A, Rodriguez-Feo JA, Sanchez dM, Rico L, Casado S. Role of nitric oxide in the control of apoptosis in the microvasculature. Int J Biochem Cell Biol. 1998;30:1095-1106.

62. Nyberg P, Xie L, Kalluri R. Endogenous inhibitors of angiogenesis. Cancer Res. 2005;65:3967-3979.

63. Vogel T, Guo NH, Krutzsch HC, Blake DA, Hartman J, Mendelovitz S, Panet A, Roberts DD. Modulation of endothelial cell proliferation, adhesion, and motility by recombinant heparin-binding domain and synthetic peptides from the type I repeats of thrombospondin. J Cell Biochem. 1993;53:74-84.

64. Taraboletti G, Roberts D, Liotta LA, Giavazzi R. Platelet thrombospondin modulates endothelial cell adhesion, motility, and growth: a potential angiogenesis regulatory factor. J Cell Biol. 1990;111:765-772.

65. Bagavandoss P, Wilks JW. Specific inhibition of endothelial cell proliferation by thrombospondin. Biochem Biophys Res Commun. 1990;170:867-872.

66. O'Reilly MS, Holmgren L, Shing Y, Chen C, Rosenthal RA, Moses M, Lane WS, Cao Y, Sage EH, Folkman J. Angiostatin: a novel angiogenesis inhibitor that mediates the suppression of metastases by a Lewis lung carcinoma. Cell. 1994;79:315-328.

67. Robaye B, Mosselmans R, Fiers W, Dumont JE, Galand P. Tumor necrosis factor induces apoptosis (programmed cell death) in normal endothelial cells in vitro. Am J Pathol. 1991;138:447-453.

68. Tsukada T, Eguchi K, Migita K, Kawabe Y, Kawakami A, Matsuoka N, Takashima H, Mizokami A, Nagataki S. Transforming growth factor beta 1 induces apoptotic cell death in cultured human umbilical vein endothelial cells with down-regulated expression of bcl-2. Biochem Biophys Res Commun. 1995;210:1076-1082.

69. Nyberg P, Xie L, Kalluri R. Endogenous inhibitors of angiogenesis. Cancer Res. 2005;65:3967-3979.

70. Sano M, Minamino T, Toko H, Miyauchi H, Orimo M, Qin Y, Akazawa H, Tateno K, Kayama Y, Harada M, Shimizu I, Asahara T, Hamada H, Tomita S, Molkentin JD, Zou Y, Komuro I. p53-induced inhibition of Hif-1 causes cardiac dysfunction during pressure overload. Nature. 2007;446:444-448.

71. Giordano FJ, Gerber HP, Williams SP, VanBruggen N, Bunting S, Ruiz-Lozano P, Gu Y, Nath AK, Huang Y, Hickey R, Dalton N, Peterson KL, Ross J, Jr., Chien KR, Ferrara N. A cardiac myocyte vascular endothelial growth factor paracrine pathway is required to maintain cardiac function. Proc Natl Acad Sci U S A. 2001;98:5780-5785. 
72. Izumiya Y, Shiojima I, Sato K, Sawyer DB, Colucci WS, Walsh K. Vascular endothelial growth factor blockade promotes the transition from compensatory cardiac hypertrophy to failure in response to pressure overload. Hypertension. 2006;47:887-893.

73. Heineke J, Auger-Messier M, Xu J, Oka T, Sargent MA, York A, Klevitsky R, Vaikunth S, Duncan SA, Aronow BJ, Robbins J, Crombleholme TM, Molkentin JD. Cardiomyocyte GATA4 functions as a stress-responsive regulator of angiogenesis in the murine heart. J Clin Invest. 2007;117:3198-3210.

74. Choi YH, Cowan DB, Nathan M, Poutias D, Stamm C, del Nido PJ, McGowan FX, Jr. Myocardial hypertrophy overrides the angiogenic response to hypoxia. PLoS One. 2008;3:e4042.

75. Condorelli G, Morisco C, Stassi G, Notte A, Farina F, Sgaramella G, de Rienzo A, Roncarati R, Trimarco B, Lembo G. Increased cardiomyocyte apoptosis and changes in proapoptotic and antiapoptotic genes bax and bcl-2 during left ventricular adaptations to chronic pressure overload in the rat. Circulation. 1999;99:3071-3078.

76. Das B, Young D, Vasanji A, Gupta S, Sarkar S, Sen S. Influence of p53 in the transition of myotrophin-induced cardiac hypertrophy to heart failure. Cardiovasc Res. 2010;87:524-534.

77. Lee CL, Moding EJ, Cuneo KC, Li Y, Sullivan JM, Mao L, Washington I, Jeffords LB, Rodrigues RC, Ma Y, Das S, Kontos CD, Kim Y, Rockman HA, Kirsch DG. p53 functions in endothelial cells to prevent radiation-induced myocardial injury in mice. Sci Signal. 2012;5:ra52.

78. Sano M, Minamino T, Toko H, Miyauchi H, Orimo M, Qin Y, Akazawa H, Tateno K, Kayama Y, Harada M, Shimizu I, Asahara T, Hamada H, Tomita S, Molkentin JD, Zou Y, Komuro I. p53-induced inhibition of Hif-1 causes cardiac dysfunction during pressure overload. Nature. 2007;446:444-448.

79. Teodoro JG, Parker AE, Zhu X, Green MR. p53-mediated inhibition of angiogenesis through up-regulation of a collagen prolyl hydroxylase. Science. 2006;313:968-971.

80. Moorjani N, Westaby S, Narula J, Catarino PA, Brittin R, Kemp TJ, Narula N, Sugden PH. Effects of left ventricular volume overload on mitochondrial and deathreceptor-mediated apoptotic pathways in the transition to heart failure. Am J Cardiol. 2009;103:1261-1268.

81. Song H, Conte JV, Jr., Foster AH, McLaughlin JS, Wei C. Increased p53 protein expression in human failing myocardium. J Heart Lung Transplant. 1999;18:744-749.

82. Condorelli G, Morisco C, Stassi G, Notte A, Farina F, Sgaramella G, de Rienzo A, Roncarati R, Trimarco B, Lembo G. Increased cardiomyocyte apoptosis and changes in proapoptotic and antiapoptotic genes bax and bcl-2 during left ventricular adaptations to chronic pressure overload in the rat. Circulation. 1999;99:3071-3078.

83. Hu P, Zhang D, Swenson L, Chakrabarti G, Abel ED, Litwin SE. Minimally invasive aortic banding in mice: effects of altered cardiomyocyte insulin signaling during pressure overload. Am J Physiol Heart Circ Physiol. 2003;285:H1261-H1269. 
84. Mandl A, Huong PL, Toth K, Zambetti G, Erhardt P. Puma deletion delays cardiac dysfunction in murine heart failure models through attenuation of apoptosis. Circulation. 2011;124:31-39.

85. Liu X, Chua CC, Gao J, Chen Z, Landy CL, Hamdy R, Chua BH. Pifithrin-alpha protects against doxorubicin-induced apoptosis and acute cardiotoxicity in mice. Am J Physiol Heart Circ Physiol. 2004;286:H933-H939.

86. Shizukuda Y, Matoba S, Mian OY, Nguyen T, Hwang PM. Targeted disruption of p53 attenuates doxorubicin-induced cardiac toxicity in mice. Mol Cell Biochem. 2005;273:25-32.

87. Sano M, Minamino T, Toko H, Miyauchi H, Orimo M, Qin Y, Akazawa H, Tateno K, Kayama Y, Harada M, Shimizu I, Asahara T, Hamada H, Tomita S, Molkentin JD, Zou Y, Komuro I. p53-induced inhibition of Hif-1 causes cardiac dysfunction during pressure overload. Nature. 2007;446:444-448.

88. Shimizu I, Yoshida Y, Katsuno T, Tateno K, Okada S, Moriya J, Yokoyama M, Nojima A, Ito T, Zechner R, Komuro I, Kobayashi Y, Minamino T. p53-induced adipose tissue inflammation is critically involved in the development of insulin resistance in heart failure. Cell Metab. 2012;15:51-64.

89. Lee CL, Moding EJ, Cuneo KC, Li Y, Sullivan JM, Mao L, Washington I, Jeffords LB, Rodrigues RC, Ma Y, Das S, Kontos CD, Kim Y, Rockman HA, Kirsch DG. p53 functions in endothelial cells to prevent radiation-induced myocardial injury in mice. Sci Signal. 2012;5:ra52.

90. Kodama T, Takehara T, Hikita H, Shimizu S, Shigekawa M, Tsunematsu H, Li W, Miyagi T, Hosui A, Tatsumi T, Ishida H, Kanto T, Hiramatsu N, Kubota S, Takigawa M, Tomimaru Y, Tomokuni A, Nagano H, Doki Y, Mori M, Hayashi N. Increases in p53 expression induce CTGF synthesis by mouse and human hepatocytes and result in liver fibrosis in mice. J Clin Invest. 2011;121:3343-3356.

91. Menendez D, Inga A, Resnick MA. The expanding universe of p53 targets. Nat Rev Cancer. 2009;9:724-737.

92. Teodoro JG, Parker AE, Zhu X, Green MR. p53-mediated inhibition of angiogenesis through up-regulation of a collagen prolyl hydroxylase. Science. 2006;313:968-971.

93. Espira L, Czubryt MP. Emerging concepts in cardiac matrix biology. Can J Physiol Pharmacol. 2009;87:996-1008.

94. Weber KT. Fibrosis and hypertensive heart disease. Curr Opin Cardiol. 2000;15:264272.

95. Boluyt MO, O'Neill L, Meredith AL, Bing OH, Brooks WW, Conrad CH, Crow MT, Lakatta EG. Alterations in cardiac gene expression during the transition from stable hypertrophy to heart failure. Marked upregulation of genes encoding extracellular matrix components. Circ Res. 1994;75:23-32. 
96. Butt RP, Laurent GJ, Bishop JE. Collagen production and replication by cardiac fibroblasts is enhanced in response to diverse classes of growth factors. Eur J Cell Biol. 1995;68:330-335.

97. Souders CA, Bowers SL, Baudino TA. Cardiac fibroblast: the renaissance cell. Circ Res. 2009;105:1164-1176.

98. Zhou B, von Gise A, Ma Q, Hu YW, Pu WT. Genetic fate mapping demonstrates contribution of epicardium-derived cells to the annulus fibrosis of the mammalian heart. Dev Biol. 2010;338:251-261.

99. Long CS. The role of interleukin-1 in the failing heart. Heart Fail Rev. 2001;6:81-94.

100. Baudino TA, McFadden A, Fix C, Hastings J, Price R, Borg TK. Cell patterning: interaction of cardiac myocytes and fibroblasts in three-dimensional culture. Microsc Microanal. 2008;14:117-125.

101. Murakami M, Simons M. Fibroblast growth factor regulation of neovascularization. Curr Opin Hematol. 2008;15:215-220.

102. Weber KT. Monitoring tissue repair and fibrosis from a distance. Circulation. 1997;96:2488-2492.

103. Weber KT. Cardiac interstitium in health and disease: the fibrillar collagen network. $J$ Am Coll Cardiol. 1989;13:1637-1652.

104. Krenning G, Zeisberg EM, Kalluri R. The origin of fibroblasts and mechanism of cardiac fibrosis. J Cell Physiol. 2010;225:631-637.

105. Isoyama S, Nitta-Komatsubara $\mathrm{Y}$. Acute and chronic adaptation to hemodynamic overload and ischemia in the aged heart. Heart Fail Rev. 2002;7:63-69.

106. Vliegen HW, van der LA, Cornelisse CJ, Eulderink F. Myocardial changes in pressure overload-induced left ventricular hypertrophy. A study on tissue composition, polyploidization and multinucleation. Eur Heart J. 1991;12:488-494.

107. Banerjee I, Fuseler JW, Price RL, Borg TK, Baudino TA. Determination of cell types and numbers during cardiac development in the neonatal and adult rat and mouse. Am J Physiol Heart Circ Physiol. 2007;293:H1883-H1891.

108. Munoz-Chapuli R, Perez-Pomares JM, Macias D, Garcia-Garrido L, Carmona R, Gonzalez-Iriarte M. The epicardium as a source of mesenchyme for the developing heart. Ital J Anat Embryol. 2001;106:187-196.

109. Zhou B, von Gise A, Ma Q, Hu YW, Pu WT. Genetic fate mapping demonstrates contribution of epicardium-derived cells to the annulus fibrosis of the mammalian heart. Dev Biol. 2010;338:251-261.

110. Gittenberger-de Groot AC, Vrancken Peeters MP, Mentink MM, Gourdie RG, Poelmann RE. Epicardium-derived cells contribute a novel population to the myocardial wall and the atrioventricular cushions. Circ Res. 1998;82:1043-1052. 
111. Piera-Velazquez S, Li Z, Jimenez SA. Role of endothelial-mesenchymal transition (EndoMT) in the pathogenesis of fibrotic disorders. Am J Pathol. 2011;179:10741080.

112. Zeisberg EM, Tarnavski O, Zeisberg M, Dorfman AL, McMullen JR, Gustafsson E, Chandraker A, Yuan X, Pu WT, Roberts AB, Neilson EG, Sayegh MH, Izumo S, Kalluri R. Endothelial-to-mesenchymal transition contributes to cardiac fibrosis. Nat Med. 2007;13:952-961.

113. Arciniegas E, Frid MG, Douglas IS, Stenmark KR. Perspectives on endothelial-tomesenchymal transition: potential contribution to vascular remodeling in chronic pulmonary hypertension. Am J Physiol Lung Cell Mol Physiol. 2007;293:L1-L8.

114. Feng Q, Di R, Tao F, Chang Z, Lu S, Fan W, Shan C, Li X, Yang Z. PDK1 regulates vascular remodeling and promotes epithelial-mesenchymal transition in cardiac development. Mol Cell Biol. 2010;30:3711-3721.

115. Li JM, Mullen AM, Shah AM. Phenotypic properties and characteristics of superoxide production by mouse coronary microvascular endothelial cells. $J$ Mol Cell Cardiol. 2001;33:1119-1131.

116. Streckfuss-Bomeke K, Jende J, Cheng IF, Hasenfuss G, Guan K. Efficient generation of hepatic cells from multipotent adult mouse germ-line stem cells using an OP9 coculture system. Cell Reprogram. 2014;16:65-76.

117. Korff T, Krauss T, Augustin HG. Three-dimensional spheroidal culture of cytotrophoblast cells mimics the phenotype and differentiation of cytotrophoblasts from normal and preeclamptic pregnancies. Exp Cell Res. 2004;297:415-423.

118. Marino S, Vooijs M, van Der GH, Jonkers J, Berns A. Induction of medulloblastomas in p53-null mutant mice by somatic inactivation of $\mathrm{Rb}$ in the external granular layer cells of the cerebellum. Genes Dev. 2000;14:994-1004.

119. Forde A, Constien R, Grone HJ, Hammerling G, Arnold B. Temporal Cre-mediated recombination exclusively in endothelial cells using Tie2 regulatory elements. Genesis. 2002;33:191-197.

120. Kiermayer C, Conrad M, Schneider M, Schmidt J, Brielmeier M. Optimization of spatiotemporal gene inactivation in mouse heart by oral application of tamoxifen citrate. Genesis. 2007;45:11-16.

121. Toischer K, Rokita AG, Unsold B, Zhu W, Kararigas G, Sossalla S, Reuter SP, Becker A, Teucher N, Seidler T, Grebe C, Preuss L, Gupta SN, Schmidt K, Lehnart SE, Kruger M, Linke WA, Backs J, Regitz-Zagrosek V, Schäfer K, Field LJ, Maier LS, Hasenfuss G. Differential cardiac remodeling in preload versus afterload. Circulation. 2010;122:993-1003.

122. Leifheit-Nestler M, Conrad G, Heida NM, Limbourg A, Limbourg FP, Seidler T, Schroeter MR, Hasenfuss G, Konstantinides S, Schäfer K. Overexpression of integrin beta 5 enhances the paracrine properties of circulating angiogenic cells via Src kinasemediated activation of STAT3. Arterioscler Thromb Vasc Biol. 2010;30:1398-1406. 
123. Heida NM, Leifheit-Nestler M, Schroeter MR, Müller JP, Cheng IF, Henkel S, Limbourg A, Limbourg FP, Alves F, Quigley JP, Ruggeri ZM, Hasenfuss G, Konstantinides S, Schäfer K. Leptin enhances the potency of circulating angiogenic cells via src kinase and integrin alphaVbeta5: implications for angiogenesis in human obesity. Arterioscler Thromb Vasc Biol. 2010;30:200-206.

124. Wykoff CC, Beasley NJ, Watson PH, Turner KJ, Pastorek J, Sibtain A, Wilson GD, Turley H, Talks KL, Maxwell PH, Pugh CW, Ratcliffe PJ, Harris AL. Hypoxiainducible expression of tumor-associated carbonic anhydrases. Cancer Res. 2000;60:7075-7083.

125. Ponchel F, Toomes C, Bransfield K, Leong FT, Douglas SH, Field SL, Bell SM, Combaret V, Puisieux A, Mighell AJ, Robinson PA, Inglehearn CF, Isaacs JD, Markham AF. Real-time PCR based on SYBR-Green I fluorescence: an alternative to the TaqMan assay for a relative quantification of gene rearrangements, gene amplifications and micro gene deletions. BMC Biotechnol. 2003;3:18.

126. Giglio S, Monis PT, Saint CP. Demonstration of preferential binding of SYBR Green I to specific DNA fragments in real-time multiplex PCR. Nucleic Acids Res. 2003;31:e136.

127. Livak KJ, Schmittgen TD. Analysis of relative gene expression data using real-time quantitative PCR and the 2(-Delta Delta C(T)) Method. Methods. 2001;25:402-408.

128. LePecq JB, Paoletti C. A fluorescent complex between ethidium bromide and nucleic acids. Physical-chemical characterization. J Mol Biol. 1967;27:87-106.

129. Izumi N, Mizuguchi S, Inagaki Y, Saika S, Kawada N, Nakajima Y, Inoue K, Suehiro S, Friedman SL, Ikeda K. BMP-7 opposes TGF-beta1-mediated collagen induction in mouse pulmonary myofibroblasts through Id2. Am J Physiol Lung Cell Mol Physiol. 2006;290:L120-L126.

130. Okada H, Kikuta T, Kobayashi T, Inoue T, Kanno Y, Takigawa M, Sugaya T, Kopp JB, Suzuki H. Connective tissue growth factor expressed in tubular epithelium plays a pivotal role in renal fibrogenesis. J Am Soc Nephrol. 2005;16:133-143.

131. McMaken S, Exline MC, Mehta P, Piper M, Wang Y, Fischer SN, Newland CA, Schrader CA, Balser SR, Sarkar A, Baran CP, Marsh CB, Cook CH, Phillips GS, Ali NA. Thrombospondin-1 contributes to mortality in murine sepsis through effects on innate immunity. PLoS One. 2011;6:e19654.

132. Hamstra DA, Bhojani MS, Griffin LB, Laxman B, Ross BD, Rehemtulla A. Real-time evaluation of p53 oscillatory behavior in vivo using bioluminescent imaging. Cancer Res. 2006;66:7482-7489.

133. Kodali R, Hajjou M, Berman AB, Bansal MB, Zhang S, Pan JJ, Schecter AD. Chemokines induce matrix metalloproteinase-2 through activation of epidermal growth factor receptor in arterial smooth muscle cells. Cardiovasc Res. 2006;69:706715. 
134. Bansal K, Kapoor N, Narayana Y, Puzo G, Gilleron M, Balaji KN. PIM2 Induced COX-2 and MMP-9 expression in macrophages requires PI3K and Notch1 signaling. PLoS One. 2009;4:e4911.

135. Mauro C, Leow SC, Anso E, Rocha S, Thotakura AK, Tornatore L, Moretti M, De Smaele E, Beg AA, Tergaonkar V, Chandel NS, Franzoso G. NF-kappaB controls energy homeostasis and metabolic adaptation by upregulating mitochondrial respiration. Nat Cell Biol. 2011;13:1272-1279.

136. Moon JO, Welch TP, Gonzalez FJ, Copple BL. Reduced liver fibrosis in hypoxiainducible factor-1alpha-deficient mice. Am J Physiol Gastrointest Liver Physiol. 2009;296:G582-G592.

137. Yates B, Zetterberg C, Rajeev V, Reiss M, Rittling SR. Promoter-independent regulation of vimentin expression in mammary epithelial cells by val(12)ras and TGFbeta. Exp Cell Res. 2007;313:3718-3728.

138. Vousden KH, Prives C. Blinded by the Light: The Growing Complexity of p53. Cell. 2009;137:413-431.

139. Moorjani N, Catarino P, Trabzuni D, Saleh S, Moorji A, Dzimiri N, Al Mohanna F, Westaby S, Ahmad M. Upregulation of Bcl-2 proteins during the transition to pressure overload-induced heart failure. Int J Cardiol. 2007;116:27-33.

140. Leri A, Liu Y, Malhotra A, Li Q, Stiegler P, Claudio PP, Giordano A, Kajstura J, Hintze TH, Anversa P. Pacing-induced heart failure in dogs enhances the expression of p53 and p53-dependent genes in ventricular myocytes. Circulation. 1998;97:194203.

141. Muller P, Kazakov A, Semenov A, Bohm M, Laufs U. Pressure-induced cardiac overload induces upregulation of endothelial and myocardial progenitor cells. Cardiovasc Res. 2008;77:151-159.

142. Naito AT, Okada S, Minamino T, Iwanaga K, Liu ML, Sumida T, Nomura S, Sahara N, Mizoroki T, Takashima A, Akazawa H, Nagai T, Shiojima I, Komuro I. Promotion of CHIP-mediated p53 degradation protects the heart from ischemic injury. Circ Res. 2010;106:1692-1702.

143. Shizukuda Y, Matoba S, Mian OY, Nguyen T, Hwang PM. Targeted disruption of p53 attenuates doxorubicin-induced cardiac toxicity in mice. Mol Cell Biochem. 2005;273:25-32.

144. Zhu W, Soonpaa MH, Chen H, Shen W, Payne RM, Liechty EA, Caldwell RL, Shou W, Field LJ. Acute doxorubicin cardiotoxicity is associated with p53-induced inhibition of the mammalian target of rapamycin pathway. Circulation. 2009;119:99106.

145. Feridooni T, Hotchkiss A, Remley-Carr S, Saga Y, Pasumarthi KB. Cardiomyocyte specific ablation of p53 is not sufficient to block doxorubicin induced cardiac fibrosis and associated cytoskeletal changes. PLoS One. 2011;6:e22801. 
146. Agnoletti L, Curello S, Bachetti T, Malacarne F, Gaia G, Comini L, Volterrani M, Bonetti P, Parrinello G, Cadei M, Grigolato PG, Ferrari R. Serum from patients with severe heart failure downregulates eNOS and is proapoptotic: role of tumor necrosis factor-alpha. Circulation. 1999;100:1983-1991.

147. Rossig L, Fichtlscherer S, Heeschen C, Berger J, Dimmeler S, Zeiher AM. The proapoptotic serum activity is an independent mortality predictor of patients with heart failure. Eur Heart J. 2004;25:1620-1625.

148. Hursting SD, Perkins SN, Haines DC, Ward JM, Phang JM. Chemoprevention of spontaneous tumorigenesis in p53-knockout mice. Cancer Res. 1995;55:3949-3953.

149. Jimenez B, Volpert OV, Crawford SE, Febbraio M, Silverstein RL, Bouck N. Signals leading to apoptosis-dependent inhibition of neovascularization by thrombospondin-1. Nat Med. 2000;6:41-48.

150. Spyridopoulos I, Brogi E, Kearney M, Sullivan AB, Cetrulo C, Isner JM, Losordo DW. Vascular endothelial growth factor inhibits endothelial cell apoptosis induced by tumor necrosis factor-alpha: balance between growth and death signals. $\mathrm{J} \mathrm{Mol} \mathrm{Cell}$ Cardiol. 1997;29:1321-1330.

151. Riccioni T, Cirielli C, Wang X, Passaniti A, Capogrossi MC. Adenovirus-mediated wild-type p53 overexpression inhibits endothelial cell differentiation in vitro and angiogenesis in vivo. Gene Ther. 1998;5:747-754.

152. Teodoro JG, Parker AE, Zhu X, Green MR. p53-mediated inhibition of angiogenesis through up-regulation of a collagen prolyl hydroxylase. Science. 2006;313:968-971.

153. Assadian S, El Assaad W, Wang XQ, Gannon PO, Barres V, Latour M, Mes-Masson AM, Saad F, Sado Y, Dostie J, Teodoro JG. p53 inhibits angiogenesis by inducing the production of Arresten. Cancer Res. 2012;72:1270-1279.

154. Zou Y, Li J, Ma H, Jiang H, Yuan J, Gong H, Liang Y, Guan A, Wu J, Li L, Zhou N, Niu Y, Sun A, Nakai A, Wang P, Takano H, Komuro I, Ge J. Heat shock transcription factor 1 protects heart after pressure overload through promoting myocardial angiogenesis in male mice. J Mol Cell Cardiol. 2011;51:821-829.

155. Farhang GM, Goossens S, Nittner D, Bisteau X, Bartunkova S, Zwolinska A, Hulpiau P, Haigh K, Haenebalcke L, Drogat B, Jochemsen A, Roger PP, Marine JC, Haigh JJ. p53 promotes VEGF expression and angiogenesis in the absence of an intact p21-Rb pathway. Cell Death Differ. 2013;20:888-897.

156. Johnson TM, Hammond EM, Giaccia A, Attardi LD. The p53QS transactivationdeficient mutant shows stress-specific apoptotic activity and induces embryonic lethality. Nat Genet. 2005;37:145-152.

157. Obacz J, Pastorekova S, Vojtesek B, Hrstka R. Cross-talk between HIF and p53 as mediators of molecular responses to physiological and genotoxic stresses. $\mathrm{Mol}$ Cancer. 2013;12:93. 
158. Mandl A, Huong PL, Toth K, Zambetti G, Erhardt P. Puma deletion delays cardiac dysfunction in murine heart failure models through attenuation of apoptosis. Circulation. 2011;124:31-39.

159. Kodama T, Takehara T, Hikita H, Shimizu S, Shigekawa M, Tsunematsu H, Li W, Miyagi T, Hosui A, Tatsumi T, Ishida H, Kanto T, Hiramatsu N, Kubota S, Takigawa M, Tomimaru Y, Tomokuni A, Nagano H, Doki Y, Mori M, Hayashi N. Increases in p53 expression induce CTGF synthesis by mouse and human hepatocytes and result in liver fibrosis in mice. J Clin Invest. 2011;121:3343-3356.

160. Zeisberg M, Hanai J, Sugimoto H, Mammoto T, Charytan D, Strutz F, Kalluri R. BMP-7 counteracts TGF-beta1-induced epithelial-to-mesenchymal transition and reverses chronic renal injury. Nat Med. 2003;9:964-968.

161. Morimoto Y, Bando YK, Shigeta T, Monji A, Murohara T. Atorvastatin prevents ischemic limb loss in type 2 diabetes: role of p53. J Atheroscler Thromb. 2011;18:200-208.

162. Ramasubbu K, Estep J, White DL, Deswal A, Mann DL. Experimental and clinical basis for the use of statins in patients with ischemic and nonischemic cardiomyopathy. J Am Coll Cardiol. 2008;51:415-426.

163. Grillari J, Hohenwarter O, Grabherr RM, Katinger H. Subtractive hybridization of mRNA from early passage and senescent endothelial cells. Exp Gerontol. 2000;35:187-197.

164. Tyner SD, Venkatachalam S, Choi J, Jones S, Ghebranious N, Igelmann H, Lu X, Soron G, Cooper B, Brayton C, Park SH, Thompson T, Karsenty G, Bradley A, Donehower LA. p53 mutant mice that display early ageing-associated phenotypes. Nature. 2002;415:45-53.

165. van Empel VP, Bertrand AT, Hofstra L, Crijns HJ, Doevendans PA, De Windt LJ. Myocyte apoptosis in heart failure. Cardiovasc Res. 2005;67:21-29.

166. Dorn GW. Apoptotic and non-apoptotic programmed cardiomyocyte death in ventricular remodelling. Cardiovasc Res. 2009;81:465-473.

167. Blum A. Heart failure--new insights. Isr Med Assoc J. 2009;11:105-111. 


\section{Curriculum Vitae}

Name: $\quad$ Rajinikanth Gogiraju

Date of Birth: 29th July 1982

Nationality: Indian

E-mail: $\quad$ gogiraju@ gmail.com

Address: $\quad$ Albrecht Thaer Weg 24G, 37075, Göttingen, Germany.

\section{Education and Research Experience}

PhD in Biology (Molecular Medicine Program)

(Jan 2011- Sep 2014)

Department of Cardiology and Pulmonary Medicine,

Georg-August-University, Göttingen, Germany.

under the supervision of Prof. Dr. med. Katrin Schäfer

JRF- Taiwan International Graduate Program

(Sep 2007 - Feb 2010)

Institute of Molecular Biology, Academia Sinica,

Nankang, Taipei115, Taiwan.

under the supervision of Dr. Sue Lin Chao

JRF- Research Assistant

(Nov 2006 - Sep 2007)

Institute of Molecular Biology, Academia Sinica,

Nankang, Taipei115, Taiwan.

under the supervision of Dr. Sue Lin Chao

JRF- Research Assistant

(Nov 2004 - Nov 2006)

Center for Cellular and Molecular Biology (CCMB),

Hyderabad, India

under the supervision of Dr. Gopal Pande

PG Diploma in Bioinformatics \& Cheminformatics

(Aug 2004 - Nov 2004)

Medwin Medical research institute,

Hyderabad, India. 
Master of Science- 'Biotechnology'

(2002-2004)

Administrative Management College,

Bangalore University, Bangalore, India.

Bachelor of Science- 'Microbiology, Genetics, Chemistry'

(1999-2002)

Avanthi Degree College, Osmania University,

Hyderabad, India.

Intermediate- 'Botany, Zoology, Physics, Chemistry'

(1997-1999)

Narayana Junior College, Hyderabad, India.

\section{Secondary School}

(1996-1997)

CBSE, Chaithanya Bharathi public school,

Gandipet , Hyderabad, India.

\section{Poster Presentations:}

1. Gogiraju R, Julia H. Steinbrecher, Stephan E. Lehnart, Michael Kessel, Matthias Dobbelstein, Katrin Schäfer Importance of tumor suppressor gene p53-mediated endothelial cell apoptosis for cardiac angiogenesis and hypertrophy. Clin Res Cardiol 102: Suppl 1 (2013) (79th Annual Meeting of the German Society of Cardiology, 2013)

2. Czepluch FS, Vogler M, Kuschicke H, Meier J, Gogiraju R, Katschinski DM, Rigger J, Hasenfuss G, Schäfer K: Significance of the transcription factor Krueppel-Like Factor 4 for endothelial cell function and angiogenesis. Clin Res Cardiol 102: Suppl 1 (2013) (79th Annual Meeting of the German Society of Cardiology, 2013)

3. Czepluch FS, Vogler M, Kuschicke H, Meier J, Gogiraju R, Katschinski D, Riggert J, Hasenfuss G, Schäfer K: The transcription factor Krueppel-like factor 4 is a positive regulator of angiogenic properties in endothelial cells. Eur Heart J 34: Suppl, 1052 (2013) (Annual Congress of the European Society of Cardiology 2013) 
4. Gogiraju R, N T- Chiou, S-Y Tung, S. Lin-Chao, "Physiological role of a small RNA, sraG in E. coli" (International conference on "Regulatory RNA in prokaryotes" Harnackhaus, Berlin, Germany. 2009).

\section{Oral Presentation:}

Gogiraju R, J.H. Steinbrecher, S.E. Lehnart, M. Kessel, M. Dobbelstein, K. Schäfer Importance of tumor suppressor gene p53-mediated endothelial cell apoptosis for cardiac angiogenesis and hypertrophy. Presented in Young Investigators Award session and won 2nd place, Coronary Pathophysiology and Microcirculation, European Society of Cardiology 2013.

\section{Publications:}

1. Gogiraju R, Xingbo Xu, Magdalena Bochenek, Julia H. Steinbrecher, MSc, Stephan E. Lehnart, Michael Kessel, Elisabeth M. Zeisberg, Matthias Dobbelstein, MD, Katrin Schaefer. Endothelial p53 deletion promotes angiogenesis and prevents cardiac fibrosis and heart failure induced by pressure overload in mice. Revised version submitted to Circulation. on July 20, 2104.

2. Czepluch FS, Bernhardt M, Kuschicke H, Gogiraju R, Schroeter MR, Riggert J, Hasenfuss G, Schäfer K: In vitro and in vivo effects of human monocytes and their subsets on new vessel formation. Microcirculation. 2014;21:148-158

3. Leifheit-Nestler M, Wagner NM, Gogiraju R, Didié M, Konstantinides S, Hasenfuss G, Schäfer K. Importance of leptin signaling and signal transducer and activator of transcription3 activation in mediating the cardiac hypertrophy associated with obesity. J Transl Med. 2013;11:170.

4. Schroeter MR, Stein S, Heida NM, Leifheit-Nestler M, Cheng IF, Gogiraju R, Christiansen H, Maier LS, Shah AM, Hasenfuss G, Konstantinides S, Schäfer K. Leptin promotes the mobilization of vascular progenitor cells and neovascularization by NOX2mediated activation of MMP9. Cardiovasc Res. 2012;93:170-80. 
5. Singh K, Mogare D, Giridharagopalan RO, Gogiraju R, Pande G, Chattopadhyay S. p53 target gene SMAR1 is dysregulated in breast cancer: its role in cancer cell migration and invasion. PLoS One. 2007;2:e660. 